
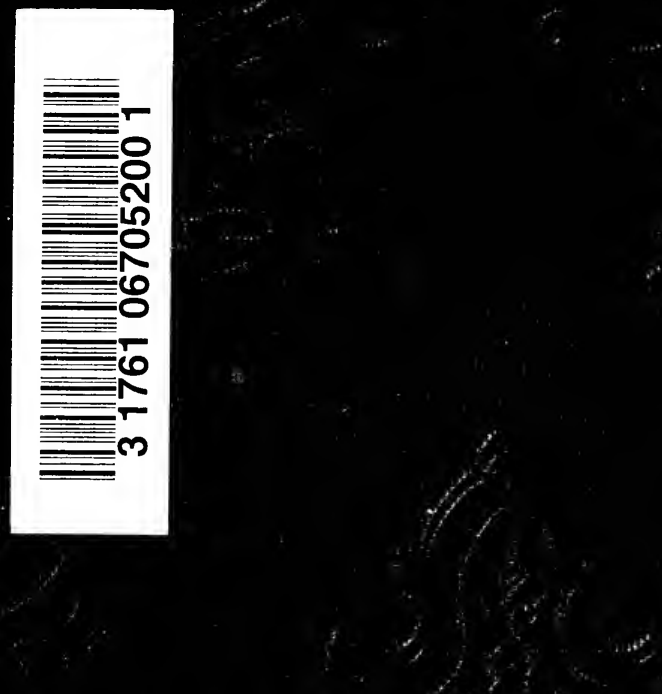
<smiles>C1CC1</smiles> 

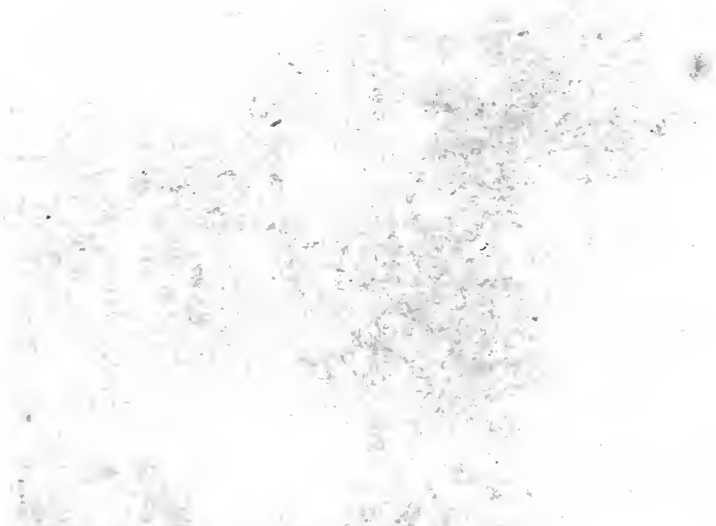

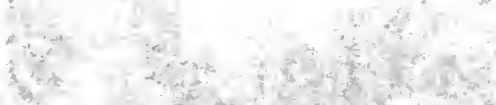

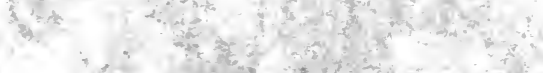

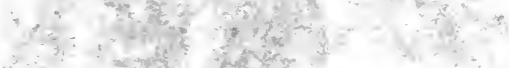

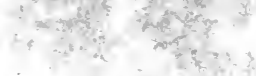

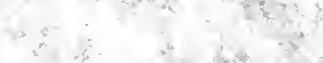

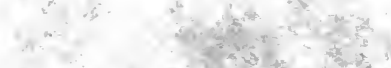

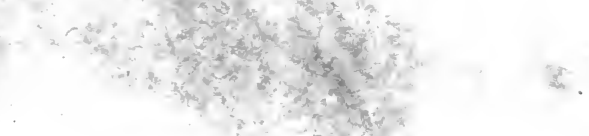

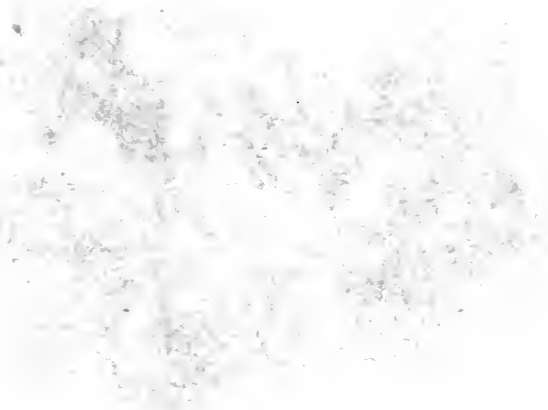


Digitized by the Internet Archive in 2007 with funding from Microsoft Corporation 




\section{ON THE \\ PRACTICE OF AUSCULTATION, ETC.}





\title{
CLINICAL IN'IRODUCTION
}

\author{
TO THE
}

\section{PRACTICE OF AUSCULTATION,}

AND OTHER, MODES OF

\section{PHYSICAL DIAGNOSIS,}

IX

DISEASES OF THE LUNGS AND HEART.

BY

\section{H. M. HUGHES, M.D.,}

FELLOW OF THE ROTAL COLLEGE OF PHXSICIANS, ASSISTANT PHXSICIAN TO GUY'S HOSPITAL, ETC.

\section{Second American,}

FROM THE SECOND AND REVISED ENGLISH EDITION.
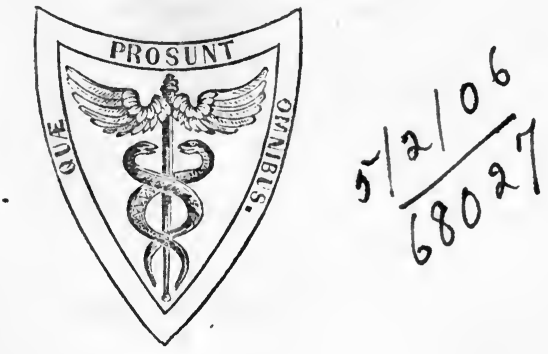

PHIL A DELPHIA:

B L A N C H A R D A N D L E A.

$$
\text { - } \quad 1854 \text {. }
$$


WM. S. YOUNG, PRINTER. 
TO HIS

FORMER CLINICAL AND WARD CLERKS,

AND TO THE

PRESENT AND FUTURE PUPILS OF GUY'S HOSPITAI, FOR WHOSE USE IT WAS MORE' ESPECIALLY WR.TTTEN,

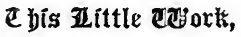

PRODUCED FROM MATERIALS PRINCIPALIY

THERE COLLECTED,

IS AFFECTIONATELY INSCRIBED,

BY THEIR SINCERE FRIEND,

THE AUTHOR. 



\section{PREFACE TO THE SECOND EDITION.}

The former edition of this little work has been long out of print; and I have been frequently urged by professors, as well as pupils, to prepare a new one. In accomplishing this, I have in no respect altered the plan, style, or general character of the book. It is still intended to ke a work for the student (whether old or young;) and to prepare, and to help him to examine for himself. It is still, therefore, simple, plain, and colloquial; and, I trust, so devoid of "erudite mystery" as to be intelligible by all.

It has been carefully revised throughout.-Some small portions have been erased; much has been, I trust, amended; and a great deal of new matter has been added; so that, though fundamentally it is the same book, it is in many respects a new work.

Dr. Stokes' recent work appeared only as it was passing through the press; and I regret that I therefore have not had the opportunity of reading his opinions on the diseases of the heart. 
Lest it should be supposed that I have unfairly neglected the consideration of the opinions of Skoda, I may state that I have been long acquainted with his views, through the paper of Drs. Drysdale and Russell, in the "Edinburgh Medical and Surgical Journal" of the year 1841; and that I have, recently, carefully examined them, as partially enunciated in the lectures of Dr. Herbert Davies, and more generally propounded in the translation of Dr. Markham. But, as I believe those views (when differing from his cotemporaries, and more especially in the very general application of the laws of consonance to diseases of the chest) to be in many-perhaps in most-respects incorrect, I have not thought it desirable to introduce any particular notice of them; fearing that it might add to the perplexity rather than to the instruction of the student.

H. M. H.

14, St. Thomas' Street, Southwark,

January, 1854. 


\section{TABLE OF CONTENTS.}

Dedication

Preface.

Table of Contents

\section{CHAPTER I. \\ Preliminary Observations and Directions}

1. Cautions as to the conduct and demeanour of Examiner $\quad 27$

2. Necessary qualifications of the Student of Auscultation $\quad 29$

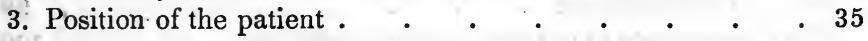

4. Divisions of the chest into regions . . . . 37

5. Different modes of Exploration in diseases of the chest $\quad 40$

CHAPTER II.

Inspection, or Ocular Examination

1. Method to be pursued, and circumstances to be noticed on Inspection

2. Indications afforded by Inspection

$\cdot \quad \cdot \quad \cdot \quad \cdot 45$

CHAPTER III.

Palpation, or Manual Examination $\quad 56$

1. Mode of employing Palpation . . • • . . . 56

2. Indications afforded by Palpation $\quad \cdot \quad \cdot \quad \cdot \quad \cdot \quad \cdot 57$ 


\section{CHAPTER IV.}

Percussion, or Examination by Striking

1. Different modes of Percussion . . . . . . . 66

1. Immediate Percussion . • . . . . 66

2. Mediate Percussion . . . . • . 68

2. Percussion in the normal state of the chest . $\quad$ - $\quad 77$

3. Natural resonance of different regions on Percussion - 78

4. Indications afforded by Percussion - $. \quad . \quad . \quad .84$

1. In diseases of the lungs and bronchial tubes $\quad . \quad \ldots 84$

Bronchitis . . . . . . . . . 84

Pneumonia $. \quad . \quad . \quad . \quad . \quad . \quad . \quad$. 84

Phthisis . . . . . . . . . 87

Venous congestion $. \quad . \quad . \quad . \quad . \quad$. 92

Edema of the lung . $\quad . \quad$. $\quad . \quad$. $\quad$. 93

Malignant disease . $\quad . \quad$. $\quad . \quad$. 93

Emphysema of the lung . . . . . . . 94

2. In diseases of the pleura $\quad . \quad$. $\quad$. $\quad$. 96

Pleuritic, or other effusion . $\quad$. . . . 96

Malignant disease, \&c. $\quad . \quad$. $\quad . \quad$. 98

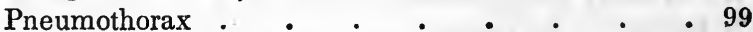

3. In diseases of the heart and pericardium . . 100

Disease of the heart . . . . . . . 100

Pericardial effusion . . . . . . . 103

4. In disease of the anterior and posterior Mediastinum, . 105

Aneurism, . . . . . . . . . 105

Abscess, \&c. . . . . . . . . 106

\section{CHAPTER V.}

Auscultation, or Examination by the Ear 108

1. Immediate Auscultation . $\quad$. $\quad$. 109

Cautions respecting it . . . . . . . 111

2. Mediate Auscultation . . . . . . . . 114

Stethoscopes . . . . . . . . . 114

3. Mode of using them . $\quad$. $\quad$. $\quad$. $\quad$. $\quad$. 121

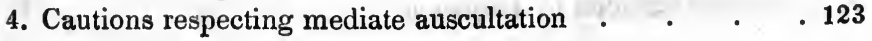


5. Combined use of immediate and mediate auscultation

6. Auscultation of the organs of respiration in health

1. Of the natural respiration $\quad . \quad$. $\quad . \quad . \quad . \quad .129$

2. Of the voice . . . . . . . . . 140

Pectoriloquy . . . . . . . . . 140

Bronchophony, \&c. . . . . . . . 141

7. Auscultation of the organs of respiration in disease . 145

1. Of the respiration in disease : $\quad . \quad$. $\quad . \quad 145$

2. Dry sounds in diseases of the air-passages and lungs .147

a. Larynx and trachea . . . . . . 147

b. Bronchial tubes . . . . . . . . . 150

Sonorous rattle $\quad . \quad . \quad . \quad . \quad . \quad . \quad .151$

Sibilating rattle $. \quad . \quad . \quad . \quad . \quad . \quad .152$

Emphysema • . . $\quad . \quad \cdot \quad \cdot \quad \cdot 153$

c. Phthisis . . . . . . . . . 157

Pneumonic consolidation $\quad . \quad$. $\quad . \quad . \quad .159$

Tubular breathing . . . . . . 160

Amphoric and fistulous breathing . . . . 161

Metallic respiration and tinkling . . . 162

d. Malignant disease . . . . . . . 164

3. Dry sounds in diseases of the pleura . . . 165

Pleuritic rubbing $. \quad . \quad . \quad . \quad . \quad . \quad . \quad .167$

Crackling . $\quad . \quad . \quad . \quad . \quad . \quad . \quad . \quad .168$

Jerking respiration $\quad . \quad . \quad . \quad . \quad . \quad . \quad 169$

Pulmonary rustling $\quad . \quad$. $\quad . \quad$. $\quad .170$

Tubular breathing . $\quad . \quad . \quad . \quad . \quad . \quad . \quad .171$

Absence of respiration . . . . . . . . 171

Exceptive cases . . . . . . . . 172

Amphoric respiration . $\quad . \quad$. $\quad . \quad$. $\quad . \quad 176$

Metallic respiration $\quad . \quad$. $\quad . \quad$. $\quad .176$

Metallic tinkling . $\quad . \quad$. $\quad . \quad$. $\quad . \quad . \quad 177$

4. Moist sounds in diseases of the air-passages and lungs $\mathbf{1 7 8}$

Comparison of moist rattles with each other . . 180

Tracheal rattle . . . . . . . . 182

Mucous rattle $. \quad . \quad . \quad . \quad . \quad . \quad . \quad 183$

Gurgling . . . . . . . . 184

Muco-crepitating rattle . . . . . . . . 186

Crepitating rattle .. $\quad . \quad . \quad . \quad . \quad . \quad . \quad .187$ 
Sounds resembling it existing in health

Moist crackling of disorganized lung

5. Moist sounds in diseases of the pleura . . . 196

Gurgling, \&c.

Metallic tinkling .. $\quad . \quad . \quad . \quad . \quad . \quad . \quad . \quad 198$

8. Auscultation of the roice and cough . $\quad$. $\quad$. $\quad . \quad 199$

1. In diseases of the air-passages and lungs $\quad . \quad . \quad .199$

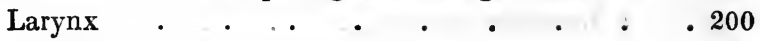

Dilatation of bronchial tubes . . . . . . 201

Emphysema . . . . . . . . . 202

Pneumonia . . . . . . . . 202

Venous congestion, \&c. . . . . . . 203

Malignant disease . . . . . . . 207

Phthisis . . . . . . . . . . 208

Pectoriloquy . $\quad . \quad$. $\quad . \quad$. $\quad . \quad$. 209

2. In diseases of the pleura $\quad . \quad$. $\quad . \quad$. 212

Ægophony . . . . . . . . . 213

Egophonic bronchophony . . . . . . . 214

Exceptive cases . . . . . . . . . 216

Absence of tractile vibration with increased vocal re-

sonance . . . . . . . . . . . 217

Metallic resonance . . . . . . . 218

9. Auscultation of the heart . . . . . . . 218

1. Auscultation of the heart in health . . . 219

a. The Impulse . . . . . . . . . . 219

b. The Rhythm . . . . . . . . . 223

c. The Sounds . . . . . . . . . . 227

The Second sound . . . . . . . . 228

The First sound . $\quad . \quad$. $\quad$. $\quad$. $\quad .230$

The natural range of the sounds of the heart . $\quad .233$

2. Auscultation of the heart in disease . . . . 235

1. The Impulse in disease . . . . . 235

2. The Rhythm in disease . . . . 243

3. The Sounds in disease . . . . . . . . . 246

a. Increase of the sounds . $. \quad . \quad .246$ 
Page

$b$. Decrease of the sounds . . . . . . 248

c. Abnormal sounds or murmurs . $\quad .250$

Direct or obstructive murmurs . . . 251

Regurgitative murmurs . $\quad$. $\quad . \quad 259$

Combined obstructive and regurgitative murmurs . • . • • • • . 262

Illustrative examples, . $\quad$. $\quad$. $\quad .263$

Anæmic murmurs . $\quad . \quad$. $\quad .270$

Venous murmurs . $\quad . \quad$. $\quad . \quad .274$

d. Pericardial rubbing, or murmur $\quad$. $\quad .279$

e. Mixed murmurs, or sounds . . . . 283

\section{CHAPTER VI.}

Mensuration, or Examination by Admeasurement 287

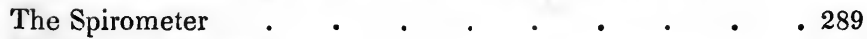
The Chest Measurer . . . . . . . . 291

I The Stethometer . . . . . . . . . 292 Mode of measuring the chest . $\quad$ - $\quad$ - $\quad$. $\quad .297$

Succussion, or Examination by Shaking $\quad 300$ Mode of effecting succussion . . . . . . . 301 




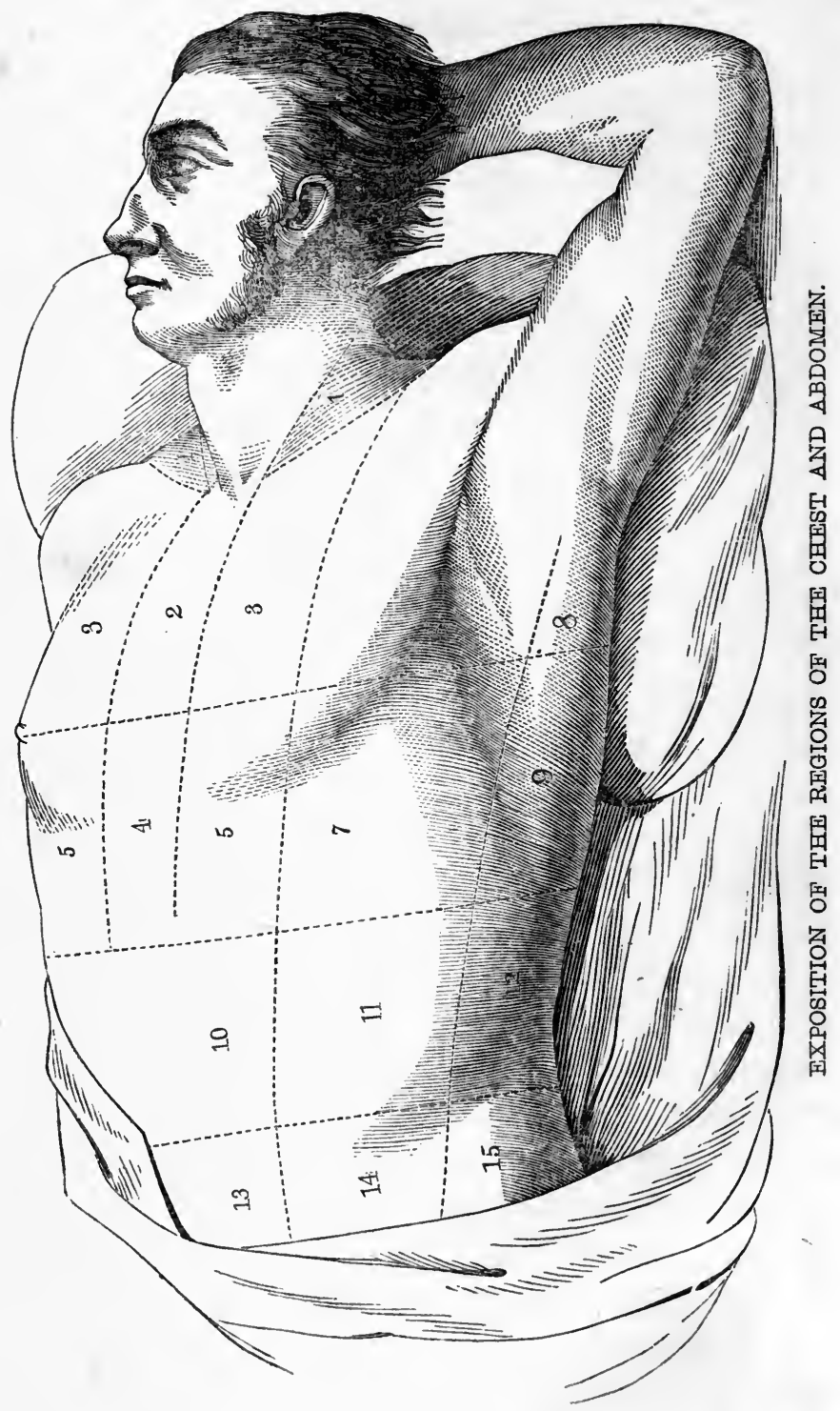




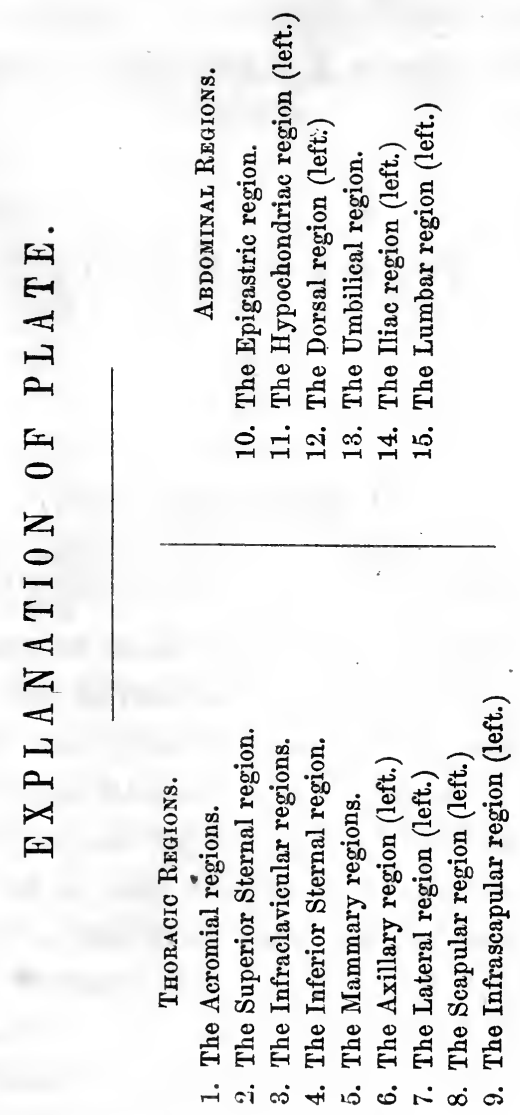





\section{AUSCULTATION, \\ ETC.}

\section{CHAPTER I.}

PRELIMINARY OBSERVATIONS AND DIRECTIONS.

IT cannot be necessary at the present day to insist upon the advantages of auscultation and percussion to the practical physician, and to every legitimate practitioner of the healing art. The remarkably rapid improvement which has recently taken place in the diagnosis of diseases of the chest, which, not more than forty years ago, were the most obscure; but which may be now considered as among the most easily recognised affections to which the human frame is liable, is almost entirely attributable to their employment. Their importance may be fairly supposed to be now generally, if not universally, conceded. It is, how. 3 
ever, as I conceive, a duty incumbent upon all who venture to write, however briefly, upon these modes of investigating disease, to endeavour to impress upon the mind of the student, what has been frequently, and in various ways, forced upon my own mind; viz. that it is, to say the least, highly desirable, if not absolutely essential to his future success in discovering and combating disease, that he should become practically acquainted with Auscultation and Percussion while at the Hospitals. To be really useful, they require much timemuch practice-much training; in other words, a prolonged education is necessary to their advantageous employment. When, indeed, facility has been previously attained, and confidence already acquired by repeated trials, and by frequent reference to the dead body, in confirmation of opinions formed at the bed-side during life, they may be used with great benefit to the patient, and satisfaction to the attendant, in private practice. But I believe that skill, and aptitude in their use, are rarely acquired in private, and that, from the comparatively limited opportunities of employing them, and of studying morbid anatomy at the homes of patients, dexterity and confidence are not often attained except in public institutions. I would therefore earnestly recommend every medical pupil at the Hospitals to become practically acquainted with auscultation, and familiar with the 
products of disease now, while he is able to do so; as I feel assured that he will never regret the time expended in the attainment of these objects. If, on the contrary, the present opportunity be allowed to pass unimproved, it is not impossible that he may never enjoy another, and it is highly probable that he never will have so wide a field, or so suitable a time, for the study of that which he will find of vast service to him in his future course.

\section{Cautions as to the conduct and demeanor of the examiner.}

Some care is occasionally necessary lest persons of an excitable and irritable temperament, whose chests are about to be explored, should be alarmed by the anticipation of the examination, the nature of which they do not understand, and the trouble and fatigue of which they consequently exaggerate. It is therefore in such cases desirable, that, if it have been previously premised that an examination of the chest is to be made, the process should be represented to be, as it really is, free from pain and attended with little inconvenience; though, if thoroughly effected, necessarily occupying considerable time. With the same view of not exciting alarm in the mind of such nervous individuals, all unnecessary display of stethoscope and pleximeter, and parade of every kind, should be avoided; otherwise some undefined notion of an 
operation may be induced, and the patient may become unwilling, or unable, to submit to the ordeal. If everything be quietly done, and all treated as a matter of course, and managed with delicacy and gentleness, I believe very few persons unless exhausted by disease, will be either unwilling without hesitation, to submit to, or unable, without injury, to bear a tolerably minute exploration of the chest.

The examiner should also endeavour at the commencement to wear a cheerful aspect, and be careful throughout not to allow the results of his investigation to escape him in the hearing of the patient, or even to permit them in any way to affect the expression of his countenance, each shade of which is sometimes watched by patients with the most intense anxiety, and even slight changes in which occasionally have a very depressing effect upon susceptible individuals.

In all examinations the benefit of the person examined should be the primary consideration.To this the mere gratification of the curiosity or interest of the examiner should be ever made to yield. The determination of a nice point of diagnosis should never be allowed to interfere with the real good of the invalid. When, therefore, patients are likely to become seriously exhausted by being examined, the exploration should not be 
attempted, or, if already commenced, should be at least temporarily suspended: unless, indeed, the immediate determination of a particular question should itself, and at that time, necessarily involve the benefit of the person examined. This caution. is more particularly necessary in hospital and other public practice, in which it sometimes becomes necessary, for the sake or the patient, and the interests of humanity itself, to restrain the ardour of pupils and other examiners.

2. Necessary qualifications of the student of Auscultation.

1. A certain amount of knowledge of anatomy and physiology is necessary to the practical auscultator. It is absolutely essential that he should be acquainted not merely with the situation and boundaries of the heart, lungs, liver, spleen, \&c., the course of the large vessels, the attachments of the diaphragm, \&c., but that he should be also well informed in the physiology of the circulation and respiration.

2. Though a thorough familiarity with the history and constitutional symptoms of disease, its morbid anatomy and pathology, is not in the first instance necessary for the successful prosecution of the study of the physical signs of thoracic disease; as both may be assuredly studied conjointly with advantage at the bed-side of the patient by even 
the tyro in medicine; yet some general knowledge of the ordinary symptoms and results of morbid processes is requsite for the student of auscultation at even the very commencement of his course. If he possess not such information, if his conclusions are derived alone from what he hears, he will find himself frequently mistaken. He may in consequence be induced to regard physical diagnosis as of little practical value, and to desist from the pursuit of that which may become to him a most important acquisition in his future discrimination of disease.

3. It is also clear that the student should have some general acquaintance, derived from reading, lectures, or clinical instruction, with the connection existing between certain sounds, and certain forms of disease. He should know, for example, that dullness on percussion, a crepitating rattle and increased resonance of the voice, usually accompany particular stages of pneumonia, and that a rubbing noise is attendant upon some forms of pleuritis and pericarditis. That he may escape the danger of forming too hasty an opinion, and avoid the error, so common with young and inexperienced auscultators, of incorrectly designating a particular sign, and consequently of misinterpreting its import, it is highly desirable that he should in the first place get pointed out to him, either by his clinical instructor, or by some more advanced pupil, a good 
characteristic example of each sign, as it occurs in practice. Of this he should endeavour to obtain a distinct and strong impression. If he obtain such an impression, he will not be likely afterwards to lose it, and to it therefore he will be able in his future investigations to refer; so that he may himself afterwards be able to say to others, as he has already been told,- "Here is a good example of pleuritic rubbing," and "there a fine specimen of crepitating rattle."

4. It is necessary that the student should have some knowledge of the more common doctrines of acoustics; such as the origin and properties of sound, the modes of its transmission, reverberation, and consonance, the diversity of the conducting power of different media, and the facility, or difficulty; with which the vibrations of sounding bodies are transmitted to others of different density, elasticity, \&c. He should recollect, what appears to have been forgotten by some writers upon auscultation, that, as regards this art, in diseases of the chest, the vibrations of sound, however originating, however conducted, and however modified before reaching the solid parietes of the chest, are transmitted either mediately by the stethoscope, or immediately to the ear by the vibrations of these solid parietes themselves; and therefore that the sound, whether originating in air, or transmitted through water, when it has reached these parietes, is in all cases 
better conducted by a solid than by any other medium; and that it is so, not merely because solids are in themselves better conductors than either fluids or gases, but because the medium of conduction is identical with that through which the sound necessarily reaches the surface of the chest.

5. It is scarcely necessary to state, that, to constitute a good auscultator, the sense of hearing must not be defective. But to become a proficient in the art of auscultation, something more is necessary than the mere absence of defect. For this purpose, what musicians call "a good ear," or a delicate appreciation of minute differences of sound, is an important, if not an essential qualification. Without it, the student may indeed be able to master marked differences; but, as I have often proved, will ever remain incapable of appreciating those delicate diversities of sound from which the accomplished auscultator mainly derives his success in diagnosis.

6. There are few attainments really valuable which can be mastered without the expenditure of much time and trouble. Auscultation and percussion are certainly not exceptions to this general rule. Patience and perseverance, then, are certainly requisite for the successful prosecution of auscultation, \&c. A long education, multiplied opportunities of examining disease, and of comparing the sounds heard during life with the revelations of the in- 
spection-room, are assuredly necessary. A caution may, perhaps, be here introduced with advantage. The student should not only, if possible ever follow the patient to the post-mortem room, should carefully mark the localities in which certain signs have been previously observed, and compare those with the condition of the subjacent parts; but he should also recollect the period of time which has elapsed between his examination and the death of the patient, and bear in mind the changes that may have occurred in the state of those parts in the interim. From a neglect of these considerations in necroscopists, I have very frequently seen students abashed and disconcerted, and the diagnosis of the physician improperly and unjustly repudiated.

7. It is essential to the successful prosecution of auscultation and percussion that their value should be fully, but at the same time fairly, estimated. If the individual employing them be influenced merely by fashion, and labour under the misconception that they are in reality of little importance to the formation of a correct diagnosis, it is highly probable that, in his hands at least, they will prove so. If, on the other hand, the student conceive that by their use he will be enabled, in every case, to determine the exact condition of a diseased organ, he will be most assuredly liable to much disappointment. They should never be, and there is no valid reason why they ever should be, employed to the 
exclusion of the other modes of investigation. When the history, and the general or constitutional symptoms of disease, have been inquired into with the same minuteness, and interest as in other cases; then, but usually not till then, should percussion and auscultation be employed, or then, at any rate, and not till then, should any deductions be made from the indications afforded by the physical signs. To a neglect, or comparative disregard of the history, and ordinary symptoms of disease, and a too implicit confidence in their highly important art, it is probable that some of the errors of even accomplished auscultators are to be attributed. There is, however, I repeat, no valid reason why the auscultator should not use with equal diligence, and be equally skilled in the employment of all other modes of investigation, as the individuals who discard the stethoscope, and make no use of the ear in forming their diagnosis, and why he should not enjoy the advantage of his art in addition to all those possessed by others. Let, then, auscultation and percussion be regarded as ancillary only to other, or rather as two among many, modes of investigating disease; as two stout and strong additional strings to our bow; albeit the information they afford is, in some cases, equal, if not superior, to that derived from all other modes combined. 


\section{Position of the patient.}

The object to be desired in reference to the position, is so to place the individual to be examined that the parietes of the chest may be put upon the stretch, without contraction of the superficial muscles, or any exertion on his part likely either to induce fatigue, or in any way to affect his perfect ease. For this purpose, when the fore part of the chest is to be examined, if the patient be able to sit up, I have found sitting upon a chair with a high, rounded, and reclining back, against which the head and shoulders can be supported without any muscular effort, to be the most suitable position. He should be placed opposite to a good light, with the shoulders thrown back, and the arms hanging down by the side of the chair. If a chair with a high reclining back be not at hand, the patient may be seated on an ordinary chair, with his arms passed over its back, his head reclined and supported by a cushion against the wall, and his shoulders as far retracted as his ease will admit. When the side, or axillary space, is to be examined, the patient's hand should be placed upon the occiput or back of the neck, and the body inclined to the opposite side. To explore satisfactorily the posterior regions, a "good back" should be made by inclining the head and bending the body forwards, and folding the arms across the breast. At the same time the scapulæ 
must be firmly pressed down by the examiner; as, by their elevation, they otherwise remove the supraand infra-spinal fossæ from those parts of the lung which they ordinarily overlie, the careful examination of which is especially important, particularly in phthisis.

Exactly the same plan may be adopted in exploring the posterior and lateral parts of the chest, if the patient keep his bed, but is able to sit thereon. If he be incapable of enduring this fatigue, he must be turned, while lying, to either side, as far as his strength and circumstances will admit. The examination of these parts, in such cases, must almost necessarily be anything but satisfactory. In the exploration of the front of the chest, on the contrary, an arrangement can generally be easily made so as fairly to examine every part without materially interfering with the supine position of the patient. This may be advantageously effected, without much trouble, by placing two pillows below the shoulders, and one only below the head, so that the former may be higher than the latter, and the chest be thereby somewhat rounded. If the position of the body be favourable, and we have the benefit of a good light thrown directly upon the front of the chest, it is not necessary that the surrounding curtains and furniture be particularly regarded; as they have really very little or no effect in modifying the sounds proceeding from that cavity in the 
process of exploration. It is, however, desirable that as little noise as possible should be made in the chamber, or ward, in which the examination is being made, and it is especially important that no part of the patient's or the examiner's dress, or any thing in contact with either, should itself, by friction, give rise to a rubbing or rustling noise, which might interfere with the purity of, or be actually confounded with, the sounds proceeding from the chest.

4. Divisions of the Chest into regions or compartments.

Opinions of the nature of diseases are materially influenced by their locality. The most important diagnostic signs are frequently, elicited by a comparison of the indications afforded by one part of the chest with those derived from another. It is often important accurately to define the limits of diseases affecting certain organs; to be able to mark their progress or their secession, or to be assured of their remaining stationary; to determine if the organs themselves are in their natural situation, or to ascertain to what extent they have diverged therefrom. It has consequently been found convenient to divide the chest, as well as the abdomen into various regions or compartments. For this purpose vertical lines passing between certain fixed points, and transverse lines passing round the body 
on a level with other fixed points, and intersecting the vertical lines, have been employed.

The following mode of dividing the chest and abdomen into regions is that generally adopted, with the exception of some slight modification of the useful additions and alterations advocated in the valuable articles, "Abdomen," and "Chest, exploration of," in the "Cyclopædia of Practical Medicine." By three vertical lines, one passing on each side of the sternum, and the other running the whole length of the dorsal and lumbar spine, the chest is first divided anteriorly into a central position, and a right and left side, and both the chest and abdomen are posteriorly divided into equilateral compartments. A vertical line is then drawn from the scapular extremity of the clavicle, on each side, to the spinous process of the pubis; another from the posterior boundary of each axilla perpendicular to the crest of the ileum, and another along the inner margin of each scapula, between two transverse lines to be immediately mentioned. The transverse lines encircle the body-1st, on a level with the clavicles; $2 \mathrm{dly}$, on a level with the junction of the cartilage of the fourth rib with the sternum; $3 \mathrm{dly}$, on a level with the extremity of the xiphoid cartilage; 4 thly, on a level with the end of the last rib; and, 5thly, on a level with the spinous processes of the ossa ilii. All these points are well defined, can be almost always distinctly 
felt, are not liable to vary (excepting from disease,) and may be, without difficulty, remembered. By these.vertical and transverse lines the chest is divided into a superior, two central, and seven other regions upon each side. The superior or Acromial region, embraces all the space between the lower part of the neck and the level of the clavicles, but is superiorly bounded behind by the seventh cervical vertebra, and before gradually tapers off or merges into another region at the upper end of the sternum. The two central regions are, the Superior sternal and the Inferior sternal. Of the other regions, two upon each side are anterior; viz. the Subclavian or Infra-clavicular, and the Mammary; two are lateral, the Axillary and the Sub-axillary, or Lateral; and three upon each side are posterior, the Scapular, the Inter-scapular, and the Sub-scapular.

The abdomen by these lines is divided into thirteen regions. Of these, nine are more or less anterior; namely, the Epigastric, the Umbilical, and the Hypogastric, which are all central ; and the Hypochondriac, the Iliac, and the Inguinal, situated upon each side of them.

Four of the abdominal regions only are posterior; viz. the right and left Dorsal, and the right and left Lumbar.

Some of these regions, which are commonly and 'properly described as belonging to the abdomen, 
are very often implicated in diseases of the chest, as the lungs descend laterally and posteriorly much below the level of the xiphoid cartilage, and the heart sometimes materially encroaches upon the epigastrium. On this account it is desirable the regional division of the abdomen should be introduced together with those of the chest. The student is presumed to be acquainted with anatomy. It is therefore not necessary, here at least, to introduce any observations respecting the particular relation of individual organs to the various regions which have been thus succinctly marked out, and the boundaries of which, so far as regards the anterior and lateral surfaces of the chest, will be sufficiently evident by a glance at the plate here introduced. The posterior boundaries are so clearly defined by prominent parts, as to require no illustration.

5. Different modes of exploration in diseases of the Chest.

The means adopted for investigating diseases of the chest by physical signs in contradistinction to the rational symptoms, are principally included under the terms Inspection, Palpitation, and Percussion, Auscultation, Mensuration, and Succussion. Upon each of these it is proposed to offer a few brief directions and observations. 


\section{CHAPTER II.}

INSPECTION, OR OCULAR EXAMINATION.

BY Inspection is intended the simple viewing the outline of the form, and the mode of working of the parietes of the chest. This mode of examination, though too often little regarded, and much too lightly performed, is certainly only secondary in importance, as a means of diagnosis, to auscultation and percussion; if indeed it may not in some cases be properly esteemed of even higher value than either of them.

1. Method to be pursued, and circumstances to be noticed, on inspecting the Chest.

In order that the contour and play of the thoracic parietes may be observed, it is obvious that the surface must be either entirely bare, or clothed with a tight elastic vest. For the efficient performance of Inspection, therefore, a certain amount of exposure, even in the case of females, can be scarcely avoided. This exposure, however, need be but very trifling. If the patient be in bed, the lower part of the thorax may be concealed by the bed-clothes while the superior regions are submitted to inspec- 
tion, and these in their turn may be covered with a light shawl or handkerchief thrown loosely across the shoulders while the inferior regions are submitted to the same process. When the patient is seated in a chair, her own garments can be easily so arranged as to perform the same office as the bed covering. If this be delicately managed, and the vestments be studiously arranged by the examiner, so as to conceal whatever is not required to be exposed, I believe that little or no difficulty will be generally experienced in acquiring, without offence to the most fastidious, all the information which Inspection is calculated to afford. It is rarely, very rarely indeed, necessary that the mammæ themselves should be exposed. The glands, if naturally developed, form so dense a covering to the portion of the parietes over which they lie, as generally to conceal the irregularities and deficiencies immediately beneath them. But it is necessary, if a competent opinion of a lady's chest is expected, that the stays should be REMOVED, not loosened, and that all bands, strings, and ties, which tend to hamper the free expansion of the chest, should be loosened.

Upon inspecting the posterior portion of the chest, it is merely necessary to place a pillow behind, or to gather the bed-clothes around, the loins, and to retain the light shawl or handkerchief across the shoulders, to obviate any appearance of indelicacy. When these arrangements have been made, the 
capacity and the general form of the chest should be first noticed. The shape of the sternum and the connected cartilages of the ribs should be observed, and any unnatural prominence, depression, shortness, or deformity in either of them should be noted. It should be ascertained if the sides be equal in size and in form: if any flattening, contraction, or prominence, exist in the one or other, or in any part of either of them, and if any fulness of the præcordial region, or any unusual separation and bulging of the intercostal spaces, or any prominence of the hypochondria, be present.

After the form of the anterior and lateral regions has been accurately observed, which may be effected almost at a glance, the movements of the chest during respiration should be examined. In the ordinary respiration of health the motions of individual parts of the chest appear, upon inspection, to be comparatively slight, in consequence of the thoracic cavity being enlarged in every direction, nearly simultaneously. They resemble the easy ebb and flow of a soft wave. When, however, a deep inspiration is taken, it is observed that the sternum is slightly but steadily projected forward; that the abdominal parietes are gently dilated; that the lower ribs are first and most considerably raised; and that the elevation of the separate ribs takes place,-(the gentle cost wave moves,) - gradually, evenly, and regularly upwards, notwithstanding 
which each and all appear to move at the same time. Every part acts separately; but each in perfect harmony with the other. At the same time, if the patient be sitting up, or lying upon his back, there may be generally noticed a gentle rising or undulation below the left breast, dependent on the impulse of the heart against the parietes. This motion of the general parietes usually occurs, in health, about eighteen times in a minute, whilst that dependent on the impulse of the heart is commonly about four times as frequent. It must be ever recollected that, from whatever cause arising, the elevation of the parietes of the chest in females, after the period of puberty, is considerably greater than that of males; that females inspire comparatively more by the elevation of the sternum and ribs, and males more by the descent of the diaphragm. Though the primary cause of this may be as obscure as the secondary one appears evident, the fact is, it is believed, universally acknowledged.

The examiner, then, should first observe the movements of the parietes in ordinary respiration; he should subsequently direct the patient to take two or three deep but slow inspirations; he should take notice if every portion of the chest be equably raised, if the ribs move freely and (if I may use the expression) independently of each other; and should be especially careful to compare the action of one side with that of the other. Posteriorly, the ge- 
neral configuration of the chest, and the mobility of the ribs, should be examined with similar attention. But in addition to the observations made upon the anterior surface, the outline of the spine should be particularly noticed, as upon the irregularities and curvatures of this bony column very often depend such contractions and other alterations in the form of the chest, as, without such notice, might not improbably be attributed to diseases of a different origin and of another kind. The discovery of a simple curvature of the spine not unfrequently leads to the satisfactory explanation of many heretofore obscure symptoms, and to the elucidation of various physical signs, which had been previously unwittingly ascribed to other causes. Every unnatural prominence, each abnormal flattening, all unusual pulsations, projections, and indentations, must be carefully observed and noted.

\section{The indications afforded by Inspection.}

When a portion of the pleura lining the ribs is affected with inflammation, the parietes connected with or overlying the part, in consequence of the pain induced by motion, are less raised during respiration than other parts of the thoracic walls. When, after the subsidence of the inflammation, the lung has, through the medium of the effused inflammatory product, become adherent to the parietes, the part affected, though it may be raised to 
an equal extent with the corresponding part upon the opposite side, is moved less freely or more stiffly than previously to the attack. It is raised as an unyielding case, rather than with the suppleness and pliancy observed between the ribs in other parts.

When, again, considerable effusion has taken place into one of the pleural cavities, from inflammation of the membrane, the lung is compressed, and a portion of the air is by such compression squeezed out; less air than in the healthy condition therefore gains admission into its cells in consequence of that compression, and the ribs therefore covering the effusion are less raised during each act of inspiration. When the fluid has been present a considerable time, and in large quantity, not only is the side, or part of the side in which it exists, immoveable during respiration, but it becomes enlarged, or misshapen; bulging at one part and contracted at another. When, again, the fluid has been at length absorbed, the side not only remains less pliable than before the attack, but, partly in consequence of the imperfect expansibility of the formerly compressed lung, already bound down by adhesions, which prevent its expansion commensurately with the absorption of the fluid, and partly in consequence of the contraction of the newly deposited fibrinous matter, it becomes flattened, or "falls in," to an extent varying in different cases. 
Hence it follows that imperfect elevation, defective pliancy, or perfect immobility of the ribs, or enlargement or contraction of one side, or of a portion of one side, become physical indications of an attack of pleurisy, or of its recent or remote consequences.

Now as pleurisy almost always coexists with a considerable deposition of tubercles, if they are near the surface of the lung; as these bodies are almost uniformly first deposited in the upper lobes, and most commonly on one side sooner, or in greater number, than on the other; as the adhesion resulting from this pleurisy interferes with the free expansion of that portion of the lung which it has invaded, and as moreover the presence of tubercles in the lung must necessarily exclude some portion of air, which would otherwise have reached the air-cells, it follows that imperfect elevation, or flattening, of one or of both infra-clavicular regions, may become one, and not unfrequently is one of the first, physical indications of tubercular phthisis.

In the more advanced stages of phthisis the indications afforded by Inspection are most striking and definite. When, for example, the upper part of the lung has become invested with a thick and -dense pleuritic coating, which is firmly united to the parietes, and when cavities have formed in the lung, and have partially collapsed, the flattening and defective mobility of the regions in which the disease exists are necessarily much 
greater. It should, however, be recollected, that phthisis may exist in its advanced, as well as in its incipient stage without this flattening and imperfect elevation of the parietes. This fact is sometimes observed in cases of very acute disease, or in those examples of the complaint in which the deposit and its consequences are almost entirely confined to the upper and posterior parts of the lung. In pneumonia, accompanied, as it usually is, with pleurisy, the indications afforded by Inspection are similar to those existing in phthisis, and dependent in a great measure upon similar causes. An important distinction, however, it must be recollected, exists in the fact that phthisis almost always first affects the upper lobes; while simple pneumonia; in a great majority of instances, attacks the lower lobes of the lungs.

When malignant disease in a massive form invades a large portion of a lung or pleura, the parietes covering it are imperfectly raised during inspiration; in consequence of a diminished quantity of air reaching the cells, and sometimes, also, in consequence of pleuritic adhesions, As the disease advances, the parietes become misshapen, either from bulging outwards, or from contraction.

In emphysema of the lungs the volume of the organ is increased, in consequence either of the dilatation of the air-cells, or much more rarely of the escape of air into the space between the lobules, or beneath the pleura. The pulmonary tissue, as 
a consequence, partially loses its natural contractility. The air is not efficiently expelled during expiration. The lungs are, in fact, more full of air than in the healthy state, and are incapable of ridding themselves of their abnormal burden. The process of the production of emphysema (independently of accidents and a few very rare cases of possibly congenital imperfection) is in general a slow and gradual one; each successive attack of bronchitis or bronchial obstruction contributes its small proportion. Every act of coughing tends to increase the general effect. In process of time the continued pressure of the lung upon the interior of the parietes effects their external dilatation, and, if the disease be general, causes them to assume a rounded form. The patient is capable of taking but little air into the lung, in consequence of the organ being already distended with air, and the parietes of the chest being already considerably dilated. Hence it follows, that a full rounded form of the parietes, together with imperfect elevation of the ribs upon inspiration, is one of the physical signs of emphysema of the lung.*

* From what has been written above, it is scarcely necessary to state that I entirely dissent from some recently propounded opinions respecting the pathology and mode of production of emphysema of the lung. Those opinions I regard not only as incorrect, but as diametrically opposed to the real state of the case. Emphysema is, I believe, the result of violent, because obstructed, expiration, not of forcible inspiration; unless it be congenital, the effect of 
In advanced or very long-continued cases of general emphysema, a notable exception to what has been stated may be observed in the lower portion of the chest. The ribs of the lateral regions are then bent inwards, and are depressed instead of being elevated; they approach to, rather than diverge from, the centre of the body, during inspiration. This appears to arise from the ribs having been already elevated, and the bony parietes permanently dilated in the upward direction to nearly the utmost extent, so that they at length become almost motionless during the inspiration. The comparatively little air which is taken into the chest gains admission, therefore, mainly by means of the diaphragm, the connexions of which muscle with the inferior ribs cause them to be drawn inwards and downwards during its action. After long continuance of this inward motion during inspiration, the ribs at the parts indicated become permanently depressed, or incurved; as in slighter and less persistent cases of emphysema they become permanently elevated, from a somewhat similar cause. This inward inclination of the lower ribs becomes very obvious upon inspection, when emphysema has existed for a long time, and the patient is already advanced in life. But, irrespectively of this incurvation of the lower ribs them-

some hereditary tendency, as in some few instances it really appears to be. 
selves, it may be often observed, in advanced cases of emphysema, that during each inspiration the lower intercostal spaces are drawn in rather than protruded. This probably arises in part from the simultaneous but abortive action of the intercostal muscles, and in part from the expansion of the lungs being insufficient to supply the space afforded by the contraction of the diaphragm. In many females the lower ribs are permanently incurved, and rendered almost motionless, by the effect of injudicious tight lacing of stays during their youth or early womanhood. It may be necessary to recollect this, lest that which is really an induced malformation should be regarded as the result of disease.

When fluid of any kind is present in the pleura in considerable quantity; if, that is to say, the complaint called hydrothorax or pneumothorax exist, the air-cells cannot be filled, the lung expanded, or the chest dilated upon inspiration, as in health. Imperfect elevation of the ribs, or total immobility of one side of the chest, therefore, become characteristic signs of these complaints.

In some cases of hydrothorax, of pneumothorax, and of pleuritic effusion, after the lung of the affected side has become compressed to the utmost, and the heart and mediastinum displaced, and also in some cases of malignant disease of the lung and pleura, the affected side of the chest becomes en- 
larged and somewhat rounded from distension. In other chronic cases of a similar nature, on the contrary, the chest becomes diminished in size and misshapen in form, either from contraction of the solid matter upon the pleura, from a wasting of the organs and muscles arising from disuse, or from partial absorption of the more watery parts of the fluid,-or perhaps in some cases from all three causes combined. In either case, the actual condition may be taken cognizance of by Inspection.

In some cases also-but most assuredly in some cases only - of fluid effusion, or of fluid combined with gaseous effusion, the intercostal spaces are elevated to the level of the ribs, and become widened and smooth, or, as it were, polished; and in some few instances they become protuberant or bulging. This latter circumstance has been considered by some high authorities as a tolerably decisive evidence that the contained fluid was pus, - that, in fact, the disease was empyema. This, as a general rule, my own observation leads me to believe, and, as a rule of universal application, convinces me is a mistake. This external configuration, however induced, may usually be observed upon Inspection.

But in many advanced cases of pleuritic effusion, of empyema, and of pneumothorax with effusion, not only, as before stated, is the side not enlarged, 
but it is, on the contrary, contracted; and not only are the intercostal spaces not widened and prominent, but they are, as is well-known by those who are practically conversant with the operation of paracentesis thoracis, actually much narrowed. This condition may be in some degree estimated by Inspection.

Herein exists a notable example of that which I am anxious constantly to impress upon the attention of the student, 一 that the results of one mode should be carefully compared with those deduced from other modes of physical examination, and the whole weighed together with the observations derived from the history, and the constitutional symptoms of the case under examination. Because the side is contracted, and the intercostal spaces narrowed, as observed upon Inspection, it might be hastily assumed, according to the dicta of many authors, that there was no fluid in the chest, while in truth the contracted side might be actually filled with serum or pus, and the other, supposed, from its greater size, to be the one diseased, might be quite healthy.

Malignant disease of the lung and pleura occasionally enlarges the side of the chest precisely as does pleuritic effusion or empyema. It is often, and particularly if originating in the pleura, accompanied with enlargement of the glands, or with tumours of the skin, or of the subcutaneous $5 *$ 
tissues. Pus in the pleura (empyema) also not unfrequently makes its way externally, and thereby forms a tumour or bulging of the external parietes: and though the nature of such tumours cannot be determined by, their presence may be ascertained, and possibly would be ascertained only, by Inspection.

When the heart has been enlarged for a considerable time, or when fluid effusion has long existed in the pericardium, a -general fulness or roundness of the præcordial region may be sometimes observed. When aneurisms of the large vessels of the heart have caused absorption of the internal parietes, or have merely become adherent thereto, an elevation, slight or considerable according to circumstances, which sometimes increases synchronously with the pulsation of the artery, may be frequently noticed upon Inspection. The consequences of fractures, penetráting wounds, or of abscesses affecting the mediastinum or the internal organs, are also commonly indicated by Inspection of the parietes.

The diseases, then, in which the diagnosis may be rendered more clear by Inspection of the chest, and in which, therefore, it may be advantageously employed, are pleuritis, and its consequences, whether in the form of simple effusion, of empyema, or of contraction, pneumonia, phthisis, emphysema, hydrothorax, pneumothorax, enlargement of the 
heart, hydro-pericardium, or other pericardial effusions, aneurism of the large vessels, fractures, abscesses, \&c.

In every primary examination, and, indeed, in all examinations in which an opinion is expected to be delivered as to the condition of the internal organs, Inspection should never be omitted. It should, however, be constantly borne in mind that curvatures of the spine, and congenital or acquired malformation of the skeleton, may so entirely change the configuration of the parietes as to cause them to present, upon mere inspection, the appearances which ordinarily coexist with almost every one of the diseases which have been mentioned.' 


\section{CHAPTER III.}

PALPATION, OR MANUAL EXAMINATION.

This mode of examination, by which the sense of touch affords its aid to the other senses in the diagnosis of disease, notwithstanding what has been said concerning the "tactus eruditus," requires comparatively little skill or experience for its exercise; so far at least as relates to the diseases of the chest.

\section{Mode of employing Palpation.}

Palpation is chiefly used in three ways. The first of these is by pressure of the sentient extremities of the fingers, as in the ordinary exercise of the-sense of touch, and requires no comment or explanation. The second mode is by placing the palmar surface of the appressed fingers, or of the whole hand, upon the part to be examined, and using such an amount of pressure as to enable it to participate in the vibrations, or to appreciate the defective motion, of the parietes of the chest, and then comparing the impressions communicated to it with those communicated to the other hand, similarly placed upon the corresponding part of the opposite 
side, or upon a different part of the same side. The third is practised by placing the sentient extremities of the fingers, or, it may be, the palm of one hand, upon the part to be examined, and gently tapping or percussing the affected part, so as to elicit a sense of fluctuation.

\section{Indications afforded by Palpation.}

When it is inconvenient to expose the chest of a patient,-when, for example, the appearance of delicacy may be considered to be thereby sacrificed - or when there may be danger of, or a liability to, an increase of symptoms by the surface of the body being bared, the appressed fingers of one hand placed flatly, and pressed firmly upon the infraclavicular region of one side, while the other is similarly placed and pressed upon the corresponding region of the other side, are often capable of distinctly appreciating a flatness of one side, or a difference in the pliability or expansibility of the two sides, in even the early stages of phthisis. During the more advanced stages of the complaint, this sign generally becomes very obvious.

When it is doubtful whether the eye has correctly measured a supposed slight difference in the action or mobility of the two sides, Palpation, exercised in the mode just mentioned, may be usefully employed to remove, or to confirm that doubt. Any considerable deficiency in the elevation of the 
ribs, or in the mobility of the parietes, or any great disparity between the two sides, is immediately detected, even without exposing the surface, by the hands placed upon the lateral regions-whether that deficiency depend upon pleuritic effusion, hydrothorax, pneumonia, pneumothorax, or any other cause. The exact nature of the complaint must be decided by farther examination of a different kind, but the fact of its existence may be ascertained by Palpation.

When disease obviously exists in one side of the chest, and the lung is clearly unexpanded from some cause or other, it is sometimes difficult to decide whether that want of expansion arise from structural disease of the lung itself, or from pressure upon its exterior, as from effusion in the pleura, \&c. In such cases assistance may be occasionally derived from Palpation; for though, when fluid exists in the pleura, the vibration of the voice may be communicated to the parietes, and may be appreciated by the ear, it is not distinguished by the hand.

The explanation of the fact appears not to be clear-but the fact itself is now acknowledged to be certain-that the vibration of the voice is communicated through the parietes to both the ear and hand when the lung is consolidated, as from pneumonia, phthisis, \&c., but that it is communicated to the ear and not to the hand, in some cases at 
least, perhaps in most cases, of effusion into the pleura. The explanation which attributes this diversity to the difference between molecular and undulatory vibrations, appears to me to be anything but satisfactory, as the facts seem to be precisely opposed to the theory by which it is professed to expound them. The most probable explanation appears to be that, though the sound may be more shrill, it is not so loud in effusion as in consolidation, and though the vibrations are more frequent, they are less strong in the former than in the latter;-that, in fact, as some vibrations which are discerned by the touch are too slow to be distinguished by the ear, so some which are obvious to the ear are too rapid to be appreciated by the sense of touch.

Whatever may be the explanation of this curious circumstance, Palpation may thereby sometimes become a means of distinguishing pleuritic effusion from consolidation or other disease of the lung, and thus afford aid in a point of diagnosis which is not always distinct even to the most experienced.

When the surface of the pleura or pericardium, which naturally glides smoothly, is roughened by solid effusion between its layers, a rubbing or grating noise is heard upon applying the ear or stethoscope to the neighbouring parts of the chest. The vibration thereby communicated to the parietes can often be perceived by the hand placed, as previously directed, upon the part affected. 
The same, with slight modifications, may be said in reference to loud noises arising from obstruction to the passage of air in the bronchial tubes, whether that obstruction depend upon thickening of the lining membrane, external pressure, or superabundant secretion. But though by Palpation the existence of these signs may be discovered, their nature, extent, and cause, must be determined by other means.

By the sense of touch the position of the heart may be generally ascertained, and, in some of its diseases, important information may be derived from manual examination. While the body is erect, the heart, when in a natural condition, is commonly felt to strike the parietes about an inch below and to the inner side of the nipple. While lying upon the back its impulse is greatly decreased, and is usually felt somewhat nearer to the sternum. When the body is turned to the left side, the impulse is felt in a direct line with, or often nearly an inch to the outer side of a line passing vertically over the nipple; while, on the contrary, when the body is turned to the right side, it is felt between the cartilages of the ribs, close to the sternum, or sometimes cannot even be felt at all. These changes are stated not to take place when the pericardium is adherent, and their absence, therefore, is assumed to be diagnostic of that condition. But this fact I have myself never had an opportunity of verifying in simple ad- 
herent pericardium without enlargement of the heart, though, when the organ is enlarged, it is well known that the exact position of the impulse is sometimes not clearly defined; inasmuch as it becomes more extensively diffused than in the normal state.

When the parietes of the heart are thickened, and the force of its impulse is consequently increased, the hand placed over the præcordial region becomes at once sensible of its abnormal force, though the pulse at the wrist may at the very same time be small and feeble.

When the cavities of the heart are dilated, with or without any increase of the thickness of their walls, the impulse is often perceptibly extended over a larger space than natural, and may be felt not only above, below, and around its ordinary site, but also in the scrobiculus cordis, and sometimes even on the right of the sternum. It must, however, be recollected that in nervous and excitable persons of spare habit, the impulse of the heart is often very extensively diffused, even when no disease of the heart exists; and therefore that a widely extended or diffused impulse is by no means a proof of the existence of disease in the heart, or in any other organ.

When the heart is removed from its natural situation by gaseous or fluid effusions into the pleura, by tumours, abscesses, \&c., it is by manual 
examination that the fact can generally be best determined.

When obstruction exists in the valves, a trembling motion, or "purring tremor" ("frémissement,") is frequently communicated to the hand, and the tumultuous action, or tumbling motion, existing in the more advanced stages of disease of the heart, and when large effusion has taken place into the pericardium, can often be best appreciated by Palpation. This subject will, however, be treated more at large in the chapter on Auscultation, when speaking of diseases of the heart. The pulsations of aneurisms, the separation or approximation of the ribs in emphysema, pleuritic effusion, empyema, hydrothorax, and pneumothorax, the seat of abscesses-whether in the parietes of, or proceeding from within, the chest-and the nature and origin of tumours, are frequently most correctly ascertained by means of Palpation. Thus by its means, the soft fluctuating feel of an abscess, or of an empyema, making its way through the parietes, may be distinguished from the solid, though it may be elastic, feel of a malignant or other tumour. By Palpation, also, when fluid exists in the pleura, and the ribs are widely separated, and the intercostal spaces are widened, and, it may be, even prominent-by placing the fingers upon those widened spaces, and directing the patient to cough, a sense of fluctuation may be sometimes appreciated. This sign, however, is very deceptive, 
and liable to lead to error. It should, therefore, never be trusted to, unless after minute comparison with the signs elicited from the opposite side, submitted to a similar process of examination. In some few cases - but, after multiplied observations, I am convinced that they are very few indeedwhen the intercostal spaces are widely separated in consequence of the pleura being full of fluidby placing the fingers of one hand upon one of the enlarged spaces, and gently tapping the same, or, indeed, other spaces, by the points of the fingers of the other hand, a sense of fluctuation may be elicited, such as is communicated to the hand in ascites or ovarian dropsy. This, however, I repeat, is very rare, and must by no means be expected as a necessary indication of the existence of fluid in the pleura. But, in the case of empyema making its way externally; or in the case of other abscesses contained within the chest, a sense of fluctuation is often communicated to the fingers placed upon the suspected tumour, when the patient is made to cough, and a gentle wave is sometimes comnicated to it by the act of respiration, and sometimes even by the impulse of the heart. It rises, that is to say, with the expiration, and falls with the inspiration: or it may have a delicate fluctuating motion communicated to it by each impulse of the heart. Palpation, therefore, may become an important means of deciding whether an abscess is inside or outside of the chest. 


\section{CHAPTER IV.}

PERCUSSION, OR EXAMINATION BY STRIKING.

Percussion is the art of striking the parietes of the cavities of the body in such a manner as to enable the examiner to judge of the amount of resonance or dulness of the parts beneath, and is one of the most important means of physical diagnosis in diseases of the chest. When the bony parietes of the chest are smartly struck by the knuckles, or by the tips of the appressed fingers, the resulting sound varies according to the nature of the organ, or the part of the organ, which lies under the portion of the parietes which is struck. The healthy lung is a light spongy tissue, the cells of which, and the tubes leading to which, are occupied by air. If the ribs, then, be struck over a portion of this tissue, when in a healthy condition, the contained air, like that within a cask or drum, resonates with the parietes; or, in other words, by its elasticity and compressibility, allows such an amount of free vibration of the parietes as to produce a moderately hollow sound. If, on the other hand, the chest be struck over a portion of the 
lung from which the air has been excluded, either by pressure from without, as in pleuritic effusion, or by deposit within, as in pneumonia or pulmonary apoplexy, then, in consequence of the parts below resonating only very imperfectly with the parietes, from defective compressibility and elasticity preventing their free vibration, a dull, heavy, or dead sound is produced-as in a cask filled with water, or a drum with dough. When the portion of the parietes which covers the heart is similarly struck, the resulting sound is also dull, in consequence of the slight amount of compressibility and elasticity possessed by that organ and its contained fluid. When, therefore, the heart is considerably enlarged, and a larger portion of the organ is in consequence uncovered by the compressible and elastic lung; when its investing membrane is distended with effused pus or serum; or when its principal vessels are greatly enlarged by aneurism, the extent of dulness in the præcordial region is increased. These few examples will suffice to illustrate the great importance of percussion in the diagnosis of diseases of the chest. The practice of this mode of exploration, apparently so simple, requires great nicety, tact, and delicacy, and considerable experience, for its efficient application. Great care is also required, in its exercise, that the examiner be not misled and deceived by trifling circumstances connected with the manner in which 
it is performed, or with the comparative tension or relaxation of the parietes of the patient.

\section{Different Modes of Percussion.}

Percussion may be either mediate or immediate. It is immediate, when the striking body, whether it be the hand of the examiner, or any other instrument, falls directly upon the parietes of the chest, or when some article of clothing only intervenes between them and the percussing body. It is mediate, when some solid material, as a disc of wood or ivory, a piece of leather, or the finger of the left hand, is interposed between the parietes and the striking body for the purpose of increasing or modifying the sound, or preventing any uncomfortable feelings of the patient.

1. Immediate Percussion may be performed with any suitable instrument, as the end of a stethoscope, or a hammer of India-rubber, \&c., with the knuckle of the contracted forefinger of the right hand; but it is most efficiently practised with the points of the appressed fingers. The extremities of the fore, the middle, and the ring finger, should be pressed close together and, by bending the first and second joints, be brought as nearly as possible to a level surface, beyond which the nails should not extend, and to which they should scarcely reach. The patient being in the position pointed out in a pre- 
ceding chapter, and the parietes being thereby rendered tense, the knuckle of the fore-finger, or the points of the appressed fingers arranged as just explained, should be smartly struck, in a direction perpendicular to the surface, successively upon the sternum, clavicle, the ribs, the scapulæ, and, though it be with less effect, the intervening soft parts. The corresponding region of the two sides should be percussed immediately after each other, so that the resulting sounds may be accurately compared; as dulness, not absolute only, but comparative, often affords most important indications. The entire chest should be thus passed under review, the examiner recollecting the natural dulness, but at same time marking the extent of the dulness of the inferior sternal and left mammary region dependent upon the heart. It may be well to state that the tips, the actual bony extremities, of the fingers should be the parts coming in contact with the chest, and not the soft bulbous sentient palmar surfaces of the last phalanges, otherwise the sound will not be so clear, as the percussing instrument will not be so firm, and therefore not so well calculated to elicit it. The examiner should be especially careful that the fingers, or other percussor employed, strike the chest at a right angle with the surface struck; as, if they fall upon the parietes in a direction only slightly oblique, the effect will be greatly diminished. 
2. Mediate Percussion.-Direct or immediate percussion may be sometimes employed with advantage, particularly when great nicety of discrimination is not required, and when it is not necessary that the examination should be prolonged. It is, however, when extensively employed, open to some and not slight objections. When, for example, the patient is an irritable or nervous person, each stroke of the fingers or percussor causes excitement, and the frequent repetition of the slight tappings upon the unprotected chest induces a state of the circulation and respiration entirely opposed to that calm and quiet condition which it is so desirable should be preserved during the future stages of the examination. When the patient is thin, or the skin delicate, the direct percussion of the slightly covered osseous structures causes inconvenience, sometimes amounting to actual pain. When the individual, on the other hand, is fat, or very muscular, when the integuments are cdematous, or when, in the female, the mammary glands are large and full, or loose and pendulous, direct percussion cannot be employed with advantage, in consequence of the dense and non-elastic soft tissues preventing the stroke of the finger reaching the bony parietes with sufficient force and sharpness to induce effectual vibrations. There are also some parts in which, from the absence of bony covering, immediate percussion is almost entirely useless. 
On these accounts, and perhaps some others, mediate percussion has been employed, and a variety of instruments have been proposed for its performance. Thin discs of wood, ivory, cork, and Indiarubber, with and without different kinds of tongues or lips to be used as handles, have been introduced and advocated under the name of Pleximeters, for the purpose of receiving and conveying the stroke of the finger, or of a variety of little hammers, under the title of Plessors. Each of these may possess some advantages, and each has had something and some one to recommend it for general employment; but each participates more or less in the objection of itself giving rise, when struck, to a sound which interferes with that dependent on the vibration of the thoracic parietes.

If any substance intervene between the striking instrument and the chest, that is, if any pleximeter be employed, it is clear that its vibratory power should be as similar as possible to that of the vibrating parietes; that it should be composed of materials identical with them,-should in fact be made of bone covered with skin. Such materials we have already prepared. A pleximeter of bone, covered with skin admirably adapted for the purpose, and always ready for use, we possess in one of the fingers of the left hand. This pleximeter possesses many advantages in addition to those already mentioned. 
It is not open to the objection of sometimes causing pain by firm pressure upon fat and thin persons, as are the wood or ivory discs which are occasionally employed. It is capable of being much more nicely adjusted to uneven surfaces than either these rigid substances, or stiff leather, and can be much more easily maintained in the required situation than flexible but elastic India-rubber. In thin persons the intercostal spaces form hollows which cannot be filled by unyielding pleximeters ; air consequently intervenes between them and the parietes, and by its resonance, when the pleximeter is struck, modifies the sound dependent on the vibrations of the instrument itself, and thereby adds to the sources of fallacy thence arising. The stiff pleximeters, moreover, cannot be conveniently applied in the axillæ, or, in very thin persons, above, or immediately below the clavicles. The firm pressure of the fingers, on the contrary, causes no pain; they are capable of being perfectly adapted to the surface of the chest, however uneven; they are therefore equally efficient as pleximeters in fat and thin persons; they can be applied in the hollow of the intercostal spaces; they can be employed in every part of the chest; when struck, they give rise to very little independent sound, and they are always at hand ready for use. On these several accounts it is believed that the very best pleximeter is produced by one of the fingers of 
the left hand, and that the very best percussor is produced by the appressed fingers of the right hand. They possess the advàntages of all others, and some which are peculiarly their own.

Having, then, decided upon the Instruments to be employed in Percussion, the next question to be considered is the mode of employing them. The fingers of the right hand being arranged as before mentioned, the palmar surface of the fore, the middle, and the ring fingers (one or all) is to be equably placed and firmly pressed upon the part under examination. If the whole chest is to be submitted to the operation, it is convenient to begin above and to pass regularly downwards. The fingers should be so firmly pressed as to make them as much as possible a part of, or one with the parietes, so that the impulse given to the fingers may be directly communicated to the parietes, and that the vibrations of the latter may be correctly appreciated by the former. It is essential that the whole extent of the finger or fingers should be equally and firmly pressed upon the surface of the chest. If the pressure be unequal, and particularly if one portion of the finger struck be in contact, and another portion not in contact with the skin, either a very imperfect sound will be elicited upon percussion, or a noise similar to that produced by striking a cracked earthen vessel ("bruit de pôt fêlé") will be brought forth. The points of the 
three appressed fingers of the right hand are now to be several times smartly and forcibly tapped upon the middle phalanx of one of the fingers of the left hand thus carefully placed, and, after the impulse is communicated, to be immediately withdrawn from contact with it.

After the sound elicited by the Percussion of one region has been thus cautiously elicited, and carefully observed, the fingers of the left hand should be removed to, and similarly placed upon, the corresponding region of the opposite side of the chest, and the sounds caused by Percussion upon each region, and every part of each region of one side, should be compared with those proceeding from those of the other side. When the præcordial space and the hypochondriac regions are percussed, this comparison of the two sides of the chest is of course not available. I have said that the stroke should be directed to, and concentrated upon, the middle phalanx of one finger. Which finger this may be is perhaps a matter of indifference; but it is better for the sake of the skill and the aptitude acquired by habit, always, excepting in particular circumstances, to employ the same finger. The middle finger is that which I myself always employ.

In mediate, as in direct, percussion the direction ef the stroke must be perpendicular to, or at right angles with the surface to which it is applied; otherwise much of the effect will be lost, and incorrect 
conclusions may be the result. If any space or spot be found, either unusually dull, or particularly resonant, upon either side, the examiner should first bring to mind, as far as he is able, the natural amount of resonance of that particular part. If in the normal condition it be similarly dull, or resonant, as the space over the heart and liver in the one case, and that covering the stomach in the other, he must attempt to define the extent, and, as far as possible, the unusual degree of dulness or resonance, and then mentally compare it with that existing in the healthy well-formed chest, to which he ought to be able to refer as to a standard, and with the sounds of which he should therefore be perfectly familiar.

It must, however, be acknowledged, that the assumed standard itself is exceedingly variable, and that it is quite impossible to define exactly the amount and extent of dulness in the healthy condition. In two individuals equally free from any indication of disease, and equally well formed, the extent of the præcordial dulness, for example, may vary considerably. An approximation to a healthy standard, however, may be attained by practice, and with this standard the comparison must be made.

If, on the contrary, the part found to be dull, ought, in the natural state, to be moderately resonant, it should be first ascertained if there exist 
any cause of dulness exterior to the chest. If none be discovered without, and it be determined that the cause exists within, the examiner should next attempt to decide whether it arise from solid or fluid material, and whether it be deep, or superficial, or both. This latter particular is effected by varying and regulating the force of the impulse communicated by the stroke of the fingers. Thus, if between a solid organ and the parietes a thin layer of healthy lung intervene, a slight and delicate stroke, or tapping, will elicit the resonance afforded by that layer, while firm pressure and a heavy stroke will detect the dulness caused by the deeply-seated solid body. Conversely to this, if the source of the dulness be only superficial, a light stroke will afford little or no sound, and a firm and strong one will afford proof of the presence of a resonant substance below.

These opposite conditions may be fairly illustrated by first percussing the lower part of the right mammary and lateral regions,' which are naturally resonant when only light, and dull when strong, percussion is employed; and then similarly examining the scapular regions of a thin person, in which an exactly contrary state of things naturally exists.

Beginners, and other inexperienced persons, are generally in the habit of giving much too strong and firm a stroke while exercising Percussion; 
forgetting, or not being aware, that to distinguish minute differences, or delicate shades in the quality, as well as the amount, of resonance-as, for example, in the comparison of that elicited from the subclavicular regions in the early stage of phthisis - light Percussion is far more efficient than the heavy strokes which are frequently employed. A gentle tap will often display a marked difference in the two sides, when a strong blow will be utterly inoperative for the purpose. If the middle finger, laid flat upon the parietes, be slowly passed down in parallel lines from the top to the bottom of the chest, and gently, but firmly, tapped as it is passed along, it is often remarkable how easily a comparatively dull space will be discovered, and how clearly it may be pointed out, even to the uninitiated, if possessing even a moderate delicacy in the appreciation of sounds.

When the source of the dulness has been proved to be superficial only, or superficial as well as deeply seated, the examiner should endeavour to ascertain if the material causing the dulness be fluid or solid. This may be sometimes, but certainly not always, determined by combining Palpation with Percussion. In other words, it is effected by estimating the amount and character of the resistance communicated by the parietes of the chest to the finger of the left hand at the time the stroke is inflicted by those of the right hand. Thus, if the cause of the dulness be a 
solid growth, the resistance afforded by the parietes is greater, and the impressibility, if I may so term it, or the resiliency, less than if it have a fluid origin. If, on the contrary, the source of the dulness be fluid, in small quantity, the resistance is less, and the resiliency greater, than when it proceeds from a solid material. But if the parietes of the chest become either locally or generally distended, and put upon the stretch, from the accumulation of fluid, then the resistance is quite as great as if it arise from a solid body. The amount of this resistance of the parietes under percussion, dependent upon the nature or the amount of the cause producing it, can be better estimated by the finger than by any other pleximeter. This forms an additional reason for its preference to any other. The objection that it causes pain to the operator himself, has some slight force, when many patients are to be continuously examined, as I have myself sometimes experienced: but the inconvenience is onIy temporary, as the phalanx of the finger upon which I have been percussing for twenty years and more is as free from pain or morbid growth as any other. The capability, however, of appreciating this difference of resistance, which is merely one of degree, is not likely to be attained but by long habit and experience, and cannot always be appreciated by the most skilful of auscultators. Fortunately there are other means of determining this sometimes important, but often difficult question. 
2. Percussion in the normal state of the Chest.

The terms used in Percussion need be only very few and very simple. For ordinary occasions and common circumstances, the words natural or normal, dull, resonant or clear, and tympanitic, appear to be amply sufficient. For though when full is opposed to empty, clear to dull, tympanitic to non-tympanitic, and high to low, there is no difficulty in appreciating the distinction; yet, when an attempt has been made by words to explain the difference between empty and clear, or high, so far as regards the results of Percussion, it has, as it appears to me, signally failed. For the student and learner, therefore, I think it far preferable to confine his attention to common terms and well-recognised distinctions.

To be capable of judging accurately of the sounds which are morbid, it is clearly necessary that the examiner should be familiar with those which are natural, and therefore that he should be conversant with the amount of resonance on Percussion in the different regions of the chest in persons free from disease. It varies, however, considerably in different individuals, quite independently of shape or congenital malformation. This difference of resonance on Percussion in healthy persons depends upon a variety of circumstances; among which may be enumerated the size of the chest, the thickness 
and looseness of the skin, the presence, absence, and amount of fat below the integuments, the development and firmness of the muscles, the elasticity of the parietes, and the general tonicity of the tissues. Still, when the internal organs are healthy, and when the external parts are natural in their congenital formation, when they are not covered with a thick layer of fat, and are not infiltrated with serum, blood, or air, and when the bones and cartilages have not been materially affected by belts, stays, or other articles of dress, the various regions bear, in regard to resonance on Percussion, a definite relation to each other, which, with a little practice, may be defined with sufficient accuracy, and with which it is absolutely essential that the student of auscultation should be acquainted.

3. Natural resonance of the different regions of the Chest upon Percussion.

Though not strictly thoracic organs, yet as organs most importantly influenced by, and influencing diseases of the lungs, it may be in the first place observed, that Percussion over the larynx elicits a clear but muffled resonance and a firm resistance, and over the trachea a clear, almost tympanitic sound, and a resilient resistance in thin persons; but that in fat people, with thick necks, these signs can be scarcely made evident. 
It has been already stated that even in health the regions of the chest vary in their amount of resonance on Percussion in different individuals. The remark is applicable to the thorax generally, but it is more especially true in reference to the acromial and some other regions. In stout persons with short necks, indeed, percussion of the acromial region affords no information, as the entire space which it embraces is uniformly dull. Even in thin persons it requires some tact and management to make the part resound. It is, however, so important carefully to examine this region, and to compare one side with the other, (particularly in reference to phthisis, which sometimes almost solely affects the very apices of the lungs, situated from an inch to an inch and a half above the lower edge of the clavicles,) that the information derived from the careful investigation of this region will amply repay the examiner for the expenditure of a little extra time and trouble. It is the neglect or superficial observation of this region which undoubtedly sometimes causes the disease to be overlooked.

To be enabled to judge of the comparative resonance of the two sides-and it is to this that the chief value of the examination is attached-the shoulders of the patient must be firmly depressed, the head thrown back, and the neck slightly inclined to the side opposite to that which is being examined. The finger of the left hand is then to 
be strongly pressed downwards, inwards, and backwards, above the clavicle, close to the bottom of the neck, and firmly percussed by those of the right hand. When the amount of resonance has been tested upon one side, the other should be similarly explored. The finger should then be removed to the top of the shoulder and similarly percussed, and should, alternately upon one side and the other, be successively passed round to each portion of the space between the scapular end of the clavicle and the spine. By careful management, depressing the patient's shoulders so as to bring the external surface as near to the lung as possible, by firm pressure and strong percussion, a considerable amount of resonance, though inferior to that of some other regions, may be brought out, and a difference in the corresponding portions of the two sides may be detected. It must be, however, repeated, that even in the most favourable circumstances the resonance of this region is defective compared with that of some other regions.

The infra-clavicular or subclavian regions are fairly resonant, excepting in stout muscular subjects, when the large pectoral muscles materially interfere with the sound on Percussion. The superior sternal region, though occupied in part by the large vessels of the heart, contains also the trachea and its primary division, as well as the approximating upper lobes of the two lungs, which cause it to be very resonant on Percussion. The 
inferior sternal region is almost always dull, from embracing a great portion of the space occupied by the heart. Superiorly, the lungs, in various degrees, overlap that organ, and on this account the upper part of this region is often resonant upon light, and comparatively dull on firm Percussion. The mammary regions vary in their amount of resonance in different parts. On the left side, over the cartilages of the fifth, the sixth, and sometimes the seventh rib, it is rather dull, particularly on firm percussion, in consequence of the left ventricle and apex of the heart being there covered with only a thin layer of pulmonary tissue. It is often on the outer side very resonant, and indeed tympanitic, nearly as high as the fifth rib, from the stomach being distended with gas, and sometimes dull to a less extent, from that organ being filled with food. On the right side the mammary region is superiorly resonant; but below the sixth rib, or the sixth intercostal space, it is dull on a forcible stroke being inflicted, in consequence of the upward projection of the diaphragm by the convex surface of the liver; though a gentle tap will easily detect the resonance arising from the intervening portion of the lung. The axillary regions, where uncovered by the converging fibres of the large pectoral muscles, and latissimus dorsi, are very resonant, as the ribs are therein covered only by the flat serratus muscle, the skin, and loose cellular 
membrane. The Lateral regions vary like the Mammary, being resonant superiorly, but inferiorly on the right side dull on forcible percussion in consequence of the underlying convex surface of the liver; and on the left side variable, according to the state of repletion or emptiness of the stomach, and the nature of its contents. The inferior and posterior part of the left lateral region is sometimes also rendered dull to a variable extent by the increased size of the spleen. When this organ and the liver are enlarged, or pushed upwards by large abdominal effusion, they, independently of any thoracic disease, materially interfere with, or entirely destroy, the natural resonance of both lateral regions.

The Scapular regions are dull, from thick layers of muscles which intervene between the integuments and the bone, and between the bone itself and the parietes of the chest. The amount of resonance, however, of the one side, may be often, particularly in thin persons, usefully compared with that of the other by vigorously depressing and rounding the shoulders, inclining the head forwards, and giving a firm strong stroke. The Inter-scapular regions are moderately resonant, excepting close to the spine, where the thick layer of muscles attached to the bony column prevents the sound being fairly brought out. By crossing the arms, and thus drawing outwards the scapulæ, and 
rendering the integuments tense, very tolerable resonance may, however, be generally elicited from these regions, excepting in very stout persons. The Infra-scapular regions are very resonant superiorly, but on the right side dull on strong percussion, and fairly sounding only upon gentle tapping below the eighth or ninth rib, in consequence of the upward projection of the liver, which in the natural condition affects the resonance in front considerably above the level at which it modifies it posteriorly. On the left side the Infra-scapular region may be rendered tympanitic by the stomach, or colon, distended with gas; or dull, either from an unusual enlargement of the left lobe of the liver, or an hypertrophied spleen; but it is usually much more resonant inferiorly than the corresponding region of the opposite side.

The different regions, independently of anydisease in the chest, but influenced by the constantly varying condition of the hollow organs of the abdomen, may be arranged as follows, according to their amount of resonance.

Tympanitic, (occasionally,) from distention of the stomach, \&c., the lower part of the left Mammary, left Lateral, and left Infra-scapular regions.

Very resonant.-The Superior Sternal, the Axillary, and the upper part of the Infra-scapular regions.

Resonant.-The Subclavian or Infra-clavicular, 
the upper part of the Mammary and Lateral, and Inter-scapular regions, excepting the inner edge of the left Mammary.

Imperfectly resonant.-The Acromial and the lower part of the right Mammary, Lateral, and Infra-scapular regions.

Dull.-The Inferior Sternal, the inner edge of the left Mammary, and the scapular regions.

4. The indications afforded by Percussion.

1. In diseases of the lungs and Bronchial Tubes.

As in simple Bronchitis, there exists merely an increased supply of blood in, together with some thickening of the lining membrane of the tubes, there is no appreciable alteration of the resonance of the chest. Natural resonance of the chest, therefore, is one of the signs of simple bronchitis. The consequences of the complaint, however, often modify the sound elicited by percussion. Thus, in the debilitated patient, pulmonary congestion, particularly of the more dependent parts of the lung, may be a direct result; and this may exist to such an extent as to be accompanied by dulness on percussion. From the long continuance, or frequent repetition of bronchitis, emphysema, dilatation of the tubes and air-cells, arises, which is attended with increased resonance of the chest.

In the early stage of Pneumonia, the pulmonary 
tissue contains a larger supply of blood than in the natural condition, and, though possibly not in the very first stage of the complaint, the air-cells and intervening spaces are, at least, partially filled with serous fluid. The ordinary quantity of air, therefore, cannot gain admission to the interior of the cells, the elasticity of the tissue is decreased, and the resonance on percussion is consequently slightly diminished. When, instead of being partially occupied by fluid, the air-cells are filled with solid or semi-solid matter, or, as the case may be, are closed by the thickening of their walls, and the air is entirely excluded from their interior, as in the advanced stages of the complaint,- - when, in fact, hepatization is established,-the dulness on percussion becomes much more marked, and the resistance afforded to the finger more decided. Of the asserted fact, that the portion of lung immediately above that consolidated by pneumonia is more resonant than natural upon percussion, I hav́e no experience. It requires confirmation by the observation of others before it can be received as established. As, in the progress of cure, the engorgement of the pulmonary tissue decreases, and the cells become partially free, the amount of dulness, together with the resistance communicated to the finger, gradually diminishes.

When, on the contrary, pneumonia passes on to the third stage, and the effused matter softens 
down, though air may gain admission to the disorganized tissue, the ressonance on percussion does not increase in proportion: excepting in the very rare cases of large pneumonic abscesses, variation in the amount of dulness is often scarcely perceptible. The comparatively slight change of the dulness in such cases probably arises from the contraction of the surrounding tissue, and the consequent depression of the ribs, as well as from the commingling of purulent or softened albuminous matter with the inspired air.

Percussion, then, forms an important element in the physical diagnosis of pneumonia. It not merely assists the physician in ascertaining the existence of the disorder, but, when the case is carefully watched, it enables him to determine its stage and progress.

Is dulness on Percussion, then, always attendant upon pneumonia?-It certainly is not. The disease may assuredly be present without any appreciable dulness. Thus, when inflammation affects separate lobules of the lung, disseminated through one or both organs, and those lobules are surrounded by a comparatively large amount of healthy crepitant pulmonary tissue, no dulness can be discovered on percussion. It is from this form of the disorder being more frequent among children, as well as from physical examination being in them effected with less facility than in 
adults, and from the patients frequently dying before the disease has arrived at the stage of consolidation, that the pneumonia of childhood is less commonly attended with dulness on percussion, than that of grown persons. There are other cases in which pneumonia cannot be recognised by dulness on percussion; as when a defined portion of hepatized lung occupies the centre of the organ, and is on every side surrounded by healthy structures, or when it exists in situations normally dull, as in the thin edge overlapping the heart, or in that passing down before the liver.

In Phthisis the dulness on percussion is very variable. In some few cases it cannot be discovered during their entire course. Its importance, like that of almost every other physical sign in this complaint, is associated with the comparison of one part with another, or of corresponding regions on the two sides of the chest. Phthisis almost always primarily affects the apex of the lungs, and most commonly that of one side, before, or to a greater extent, than that of the other side. Upon this circumstance do the distinctive physical signs of the complaint mainly depend. When, therefore, tubercles are largely and equally distributed throughout the whole of one or both lungs, the characteristic physical signs of the complaint are absent.

It has been previously stated, that when tubercles are deposited in considerable numbers in any part of the lung, the pleura covering that part al- 
most always becomes inflamed; plastic matter is effused, which connects the pulmonary and costal layers of the membrane. The expansibility of the pulmonary tissue is thereby diminished, from its free motion being impeded, and from the contraction which sooner or later ensues in the newlyformed membranous covering. The tubercles also encroach upon the space to which air is ordinarily admitted, and when in an active state, are almost always associated with more or less consolidation of the pulmonary tissue, the result of surrounding pneumonia.

From these causes less air enters the cells of that part of the lung. Hence, even in the early stages of phthisis, arises a difference of resonance in the corresponding regions of the two sides, or between different regions of the same side. The regions thus affected are almost universally the infra-clavicular, the acromial, or the scapular. There may be no actual dulness either absolute or cornparative. Both sides may sound well. But when they are accurately compared by gentle tapping, as well as strong percussion, in a compressed as well as in an expanded state of the lungs, a difference of tone or quality of the sound elicited by percussion may be often observed. This difference of tone or quality of sound, especially when existing in combination with other physical signs, should be carefully noted, as an important particular in the diagnosis of incipient phthisis. 
When tubercles become more numerous; when the pleuritic coating has increased in thickness and density; and especially when the lung surrounding the tubercles is rendered hard and firm by pneumonia; when, in fact, the part affected has become more solid from deposition of new matter, as well as from the admission of a comparatively small volume of air into the cells, the sound on percussion not only varies in tone, but differs in degree. Dulness on percussion, not only comparative but absolute, then becomes decided, but is rendered increasingly obvious by comparing the sound elicited with that produced by percussing a healthy and normally resonant part of the chest. It may exist, and equally, upon both sides, but at this period of the complaint it is most commonly present on one only, or is much more marked upon one than upon the other side.

During the stage of softening of the tubercular matter, the dulness is scarcely altered, unless it be so from an increase or extension of the surrounding pneumonic consolidation.

In the latter stages of the complaint, even when cavities have been formed, unless the vomicæ be large and superficial, the sound upon percussion is far from clear; it is often nearly, if it be not actually, dull. This arises partly from the thick and dense pleuritic covering, and the layer of consolidated lung, which intervene between the ca- 
vities and the parietes, partly from the irregular form of most vomicæ being unfavourable to the vibration of the contained air, and sometimes, in part, from the presence of fluid secretion within them.

The sound upon forcible percussion is not, however, absolutely dull, as in some of the former stages of the complaint, in the consolidation of pneumonia, or particularly in pleuritic effusion, but rather resembles that of a sounding body to which a damper has been applied, or which is enveloped by a bad conductor of sound, as when the finger is smartly filliped upon the cheek fully distended with air.

When a cavity is very large, occupying the greater part of an entire lobe of the lung, the sound may be not only more than ordinarily resonant, but absolutely tympanitic. Such cases are, however, rare, and as a general rule tubercular cavities are not, as is supposed by many, accompanied with increased resonance on percussion, but the contrary.

In a few cases a peculiar sound is heard, which has been not inaptly compared to that produced by striking a cracked pot (bruit de pôt felé.) It appears to be connected with the imperfect resonance of the air contained within a cavity of moderate size, in consequence either of its being partially occupied by fluid; of the vibration of its parietes being interrupted by the entrance of one 
or more large tubes; or of the elasticity of the parietes causing a portion of the air contained in the cavity to be pressed out at each stroke of the finger through an open tube, the noise produced by the passage of which interferes.with the sound of the air in the cavity itself. A pretty accurate, though exaggerated, representation of it may be produced by pressing the palms of the hands together, and striking the back of one of them against the knee; as when the chinking of coin is simulated for the amusement of children. It is often involuntarily induced by unskilful percussors, and may be generally elicited by placing the finger lightly, in part of its length only, upon the chest, and then smartly tapping that part of the finger which is not closely applied to the parietes. The cause of this peculiar sound in the two cases, and probably in all three, is similar if not identical.

In that form of phthisis, then, in which tubercles are generally disseminated throughout the lungs, the resonance of the chest upon percussion is often not appreciably altered. In the earlier stages, even of the more common form of the complaint, there may exist only a variation in the resonance, or rather in the tone of sound elicited by percussion, on comparing that of the infra-clavicular or acromial with that of the mammary or axillary regions of the same side, or that of corresponding regions 
of different sides with each other. In the second and third stages of the disorder,-those, namely, of consolidation and softening, - the dulness on percussion is obvious, when compared with the sound proceeding from a healthy lung. In the latter stages of the complaint the sound varies according to the size and arrangement of the cavities; it may be almost dull, it may resemble a muffled resonance, it may be tympanitic, and lastly, it may approach to that arising from striking a cracked pot.

In simple Venous congestion of the lung, arising from the difficult transmission of blood through the heart, dulness on percussion exists in a degree and to an extent proportioned to the amount and duration of the disease, and to the debility or exhaustion of the patient. In such cases the dulness is generally observed in the most dependent parts of the lungs, or in those most remote from the centre of circulation; the infra-scapular regions are therefore those which are principally affected. It is not, however, of so marked and decided a character as that arising from either pneumonic consolidation, or pleuritic effusion. But when the congestion reaches to such a height as to cause pulmonary apoplexy, in which the part affected is rendered perfectly destitute of air, and reduced to a state resembling damson cheese, the dulness on percussion, and the resistance afforded to the finger, are com- 
plete. This disease, it may be observed, often affects small portions only of the lung, or the narrow edges of the organ, and is consequently not always detected even upon minute physical examination.

In OEdema of the lung arising from diseases of the heart, or from other causes, serum partially occupies the space ordinarily filled by air. This affection, therefore, which almost always occurs in the most dependent parts of the lung, is also accompanied with a moderate amount, but, unless it be accompanied with serous effusion into the pleura, with a moderate amount only, of dulness on percussion.

In Malignant disease of the lung, the pulmonary tissue is pushed aside, and the air is entirely excluded from the parts affected by the cancerous or fungous growth. When, therefore, the disease occupies a large space, or invades a considerable amount of pulmonary tissue in the massive form, there exists perfect dulness on percussion, and great resistance to the finger.

Malignant disease, however, like scrofulous tubercles, lobular pneumonia, and pulmonary apoplexy, may exist in small rounded disseminated masses, surrounded by healthy pulmonary tissue, to a comparatively large extent. Under such circumstances, the healthy portion of the lung surrounding the malignant deposit, as in the other diseases specified, conceals the dulness which would 
be otherwise produced by it. When, therefore, malignant disease attacks the lung, in a massive form, as it usually does when originally developed therein, it is accompanied by dulness on percussion. When, on the contrary, it is distributed through the lung in the form of variously-sized tubercles, as it ordinarily is when arising secondarily to its appearance in other parts, it is often not accompanied by any appreciable alteration of the resonance of the chest.

Emphysema of the lung.-The diseases hitherto noticed have been accompanied with a decrease of the natural resonance of the chest; Emphysema of the lung produces exactly the opposite condition. This affection, as has been previously explained, is dependent upon the dilatation or rupture of the air-cells, or of atrophy, and consequent yielding, of the partitions between them. In any case, and in all cases, whether the affection be merely vesicular, interlobular, or sub-pleural, the proportion of air to the solid and fluid constituents of the pulmonary organs is increased. The lung, either locally or generally, is increased in volume, and, according to the extent and degree of the affection, loses its elasticity, and does not collapse, as in the normal state, when the chest is opened after death. When confirmed and general; the dimensions of the parietes are enlarged, and the resonance upon per- 
cussion is augmented. Not only are the parts ordinarily resonant rendered more so by this affection, but those which, in the natural condition, are dull, become well-sounding. Thus, in consequence of the enlarged lung extending over the heart, the præcordial region often becomes perfectly resonant on percussion, excepting towards the xiphoid cartilage, where, from the increased size and distention of the right side of the heart, the dulness is even greater and more extensive than natural. Thus, also, in the parts which are ordinarily dull from the underlying liver, the sound on percussion becomes comparatively resonant.

In some rare cases in which the entire lung has been consolidated, the side has been found not only resonant, but tympanitic, upon percussion. The explanation of this is uncertain, and the cause obscure. I have never seen an instance of it myself; but from the fact of a consolidated lung, and even pleuritic effusion, when situated over the stomach distended with gas, being often accompanied with even a tympanitic, though somewhat muffled, resonance, I am disposed to believe that in the cases referred to the tympanitic resonance has depended upon the vibrations of the air in a largely distended colon or stomach being excited through the solidified lung; that, in fact, it was not the lung which was resonant, but the gas in the colon or stomach. This is an entirely different case from the increased 
resonance of a lung partially compressed by effusion, though it has been confounded with it.

2. Indications afforded by Percussion in Diseases of the Pleura.

When fluid of any kind is effused into the pleura, the lung, to a corresponding extent, is compressed, and the air, in nearly an equal proportion, is squeezed out of, and prevented regaining admission to, the pulmonary cells. Whether, therefore, the disease arise from inflammation or from obstruction; whether the fluid be blood, pure serum, turbid serum or pus, dulness on percussion, as a rule exists to an extent, and, up to certain limits, to a degree also, commensurate with the amount of fluid. The dulness in either case may, and generally does, primarily affect only the lower part of the serous cavity, and gradually extend upwards as the fluid increases, and, by its increment, displaces the lung. But it may also, on the contrary, in either case only extend over a defined space, to which it is confined by previously existing pleuritic adhesions.

When the pleura is free from such adhesions, the fluid, from whatever part of the membrane it proceeds, may in each case gravitate to the lowest part of the cavity, and its site may be changed according to the varying position of the patient's body; in each case, therefore, the part in which the dulness is observed may also vary together with the changes 
of position. This change in the situation of the fluid, and of the consequent dulness, according to the position of the body, is, however, far more common in hydrothorax, than in either simple pleuritic effusion or empyema, in which diseases the fluid is much more frequently confined to a defined space by surrounding adhesions, or gravitates with less facility. Though, therefore, the change of the situation of the dulness from position may serve to distinguish that arising from hydrothorax, from that produced by pneumonia, it cannot be trusted as necessarily, or even commonly, diagnostic between pneumonia and empyema.

Even when pleuritic fluid has been removed by the use of medicine, or by the employment of paracentesis, dulness on percussion, though in a less degree, often remains for a considerable time. This probably arises from two causes, viz., the imperfect expansion of the layer of lung formerly in contact with the inflammatory effusion; and the presence of a coating of solid lymph, of variable thickness, left upon the surface of the serous membrane. When the fluid of empyema is absorbed, or is evacuated either by the trocar or by a spontaneous opening, the lung is generally so thickly covered with solid matter, so firmly bound and plastered down by bands and adhesions, as to be almost incapable of expansion, excepting after the expiration of a considerable time; and even then the expansion is often 
very imperfect. The lung of the opposite side becomes enlarged, and partially fills up the space. But it does so partially only; its supplementary action is not complete. Hence the ribs of the diseased side, from atmospheric pressure, fall in upon the space formerly occupied by the fluid, the chest becomes misshapen, and the spine, especially in young subjects, becomes more or less distorted. Hence, also, though not a single drop of fluid may be present in the pleura, though the serous cavity may be entirely closed by adhesions, dulness on percussion may remain as a consequence of empyema long after the disease itself is cured. Natural resonance may never return.

In Malignant disease of the pleura, as in that of the lung, the pulmonary tissue is pushed aside, and dulness and resistance exist on percussion commensurately with the extent of the solid deposit.

Scrofulous tubercles, when confined to the pleura, rarely exist in such numbers or to so great an extent as to give rise to dulness on percussion.

In Pneumothorax the air in the pleura pushes aside and compresses the lung to an extent varying according to the amount of the disease previously existing in the lung itself, and of the adhesions connecting it with the costal pleura. The part of the chest into which the air has escaped is generally more resonant than usual, and not unfrequently tympanitic upon percussion. When air 
and fluid coexist in the same serous sac, dulness is present at one part; and tympanitic resonance at another. The two conditions vary in extent according to the respective amount of the gas and the fluid. Under these circumstances the fluid almost always occupies the lowest part of the chest; and gravitates, according to the position of the patient, to the space which is the most dependent. The posterior will therefore be dull, and the anterior part of the chest resonant, on percussion, when the patient lies upon his back; while exactly the converse state of things will be observed, when, if able to do so, he turns upon his face.

Is, then, tympanitic resonance upon percussion a necessary accompaniment of, and always present in pneumothorax?-It certainly is not. The air may be in quantity so small, the pleura so thickly lined with solid matter, or the pneumothorax may be so centrally situated at the base of the lung, in contact with the diaphragm, and therefore so far removed from the vibrating parietes, and the intervening substances may be such indifferent conductors of the vibrations produced therein, that no increase of resonance can be elicited by percussion. The side may, indeed, be even more dull than natural; though this is of rare occurrence.

The absence of tympanitic resonance in pneumothorax is more frequent in those cases in which air enters the pleura in consequence of the partial 
evacuation of an empyema through a bronchial tube, than in those which result from the communication of a tubercular cavity with the serous membrane. In the former cases the lung has usually been already compressed to the utmost by the inflammatory effusion, and air only enters the pleura as the purulent fluid is expectorated. A portion of the space formerly occupied by the purulent effusion is also filled up by the return of the distended ribs and detruded diaphragm to their accustomed boundaries. The air in the pleura, therefore, is sometimes in quantity so small, and is so thickly surrounded by solid albuminous deposit, as not to admit of resonance on percussion. The same relative state of parts sometimes exists in pneumothorax arising from other causes, when there is a gradual accumulation of pleuritic effusion, and the comparatively heavy liquid excludes the light air from the cavity of the serous membrane. This condition is far from unfrequent in the last stages of pneumothorax connected with tubercular phthisis.

3. Indications afforded by Percussion in Diseases of the Heart and Pericardium.

In diseases of the heart.-The extent of dulness of the præcordial region varies considerably in different individuals, quite independently of disease. 
This difference arises, perhaps, in part from congenital formation, and in part from the effects of dress. The normal præcordial dulness cannot, therefore, be defined by any precise boundaries. It may be stated, as an approach to the average of its ordinary extent, that it occupies the entire inferior sternal region, and that portion of the left mammary region which is covered by the cartilages of the fifth, sixth, and seventh ribs, with the intervening intercostal spaces. If the præcordial dulness much exceed these limits, the excess may be usually correctly attributed to disease; though it by no means necessarily follows that that disease is connected with the heart, or with its investing membrane. It may arise from local pleurisy, or consolidation of the lung, from abscess of the anterior mediastinum or connected with disease of the ribs, from malignant disease or aneurism of the descending thoracic aorta. The præcordial dulness may, on the other hand, not extend to its ordinary boundaries without disease being present in any organ. It may be almost entirely cancelled by an overlying emphysematous lung, without any cause for the diminished dulness existing in the heart itself. Still further, though great enlargement of the heart may be present, it may happen that, excepting in the scrobiculus cordis, and lower part of the sternum, no dulness on percussion may be perceptible, in consequence of the emphysema- 
tous lung intervening between the hypertrophied or dilated organ and the parietes. The air in the emphysematous lung induces such an amount of resonance on percussion as to conceal the normal dulness of the part.

These circumstances being duly considered, and the exceptions borne in mind, it may be stated generally that all enlargements of the heart, and all effusions into the pericardium, are accompanied with increased dulness on percussion of the præcordial region. The cause of this must, from what has been previously stated, be sufficiently obvious; and need not be here explained. When, therefore; from any obstruction to the course of the blood existing in the aortic valves, or in the course of the thoracic aorta itself, or from any other cause, the left ventricle is greatly dilated and thickened, an increase in the extent of the præcordial dulness is observed, especially over the costal cartilages, and below the left nipple.

When obstruction exists in the mitral valve, when the auriculo-ventricular opening is contracted or admits of regurgitation, the left auricle is imperfectly emptied during the diastole, and is more than ordinarily distended during the systole, of the ventricle. The auricle at length becomes permanently dilated and thickened. This may be often ascertained by an extension of the præcordial dulness to the cartilages, and adjoining bone of the left 
fourth rib, the third intercostal space, and even the third rib.

When the lungs are extensively affected with emphysema; when, in consequence of disease in the mitral valve, the circulation of the blood through the lungs is obstructed; or when, from either congenital or acquired smallness of the calibre of, or from any other disease in, the pulmonary artery, there exists an impediment to the free transmission of the blood from the right ventricle, repletion and distention of that cavity, and of its adjoining auricle, necessarily ensue. The evidence of this exists in the extension of the ordinary præcordial dulness to the right side of the sternum, or the inner edge of right mammary region, and particularly to the scrobiculus cordis.

In Pericardial effusion.-From the difficulty, or rather the impossibility, of accurately defining the natural limits of the præcordial dulness in individual cases, the small increase of that dulness which may be presumed to be dependent on the plastic effusion of recent pericarditis is not discoverable by percussion. The presence even of fluid effusion cannot always be ascertained by percussion, unless it exist in considerable quantity.

When, however, the quantity of fluid is considerable, the dulness on percussion is perceptibly increased. If the serous membrane be free from adhesions, the increased dulness commences below, 
extends on either side, but particularly on the left side, of the lower portion of the sternum, and gradually passes upwards.

When the membrane is largely distended with fluid, the increased dulness is sometimes very remarkable and extensive. Its boundaries are enlarged in every direction, but especially transversely and inferiorly.

Whatever may be the shape of the healthy pericardium when artificially filled with fluid, it certainly does not assume the shape of a flask, as has been stated, when distended from disease. It much more nearly resembles a pig's bladder with its fundus downwards; and when less completely distended, a triangle with exceedingly obtuse and rounded angles. These facts may be in some slight degree traced by the accompanying dulness on percussion.

When partial adhesions exist in the membrane, and prevent its distention in certain directions, the fluid effused into the free portions of the serous sac causes the outline of the dulness to be irregular in form.

Hence it happens that pericardial effusions may exist in considerable amount without being distinguishable by any increased dulness; or, in other words, the dulness may so slightly extend beyond the limits naturally existing in some healthy individuals, as not to be certainly appreciated as the 
result of disease. In other cases, the dulness may be general and extensive, and in a third set of cases it may be limited and local, though at the same time definite and decided.

4. Indications afforded by Percussion in Diseases of the Anterior and Posterior Mediastinum:

When aneurism proceeds from that portion of the aorta which is just above the valves, the dulness to which it gives rise may be undistinguishable from that proceeding from disease of the heart itself. It may extend upon either side of the heart, though it is most commonly situated on the right side. Independently of the dulness arising from the tumour itself, which varies of course according to the size of the sac, the disease often displaces the heart so as considerably to modify the normal dulness of the præcordial region.

When aneurism exists in the ascending aorta, and is of large size, its presence may be generally recognised by an alteration of the resonance on percussion over the upper sternal, and the inner edge of the right subclavian region. Unless, however, the dimensions of the aneurism are considerable, no absolute dulness is perceptible, in consequence of the overlying resonant lung concealing that which the presence of the tumour would otherwise induce. 
The same may be said in reference to aneurism of the anterior innominata. But even when no dulness, strictly so called, can be discovered, there may be often distinguished a variation of the tone or quality of the sound when corresponding regions of the two sides are percussed, and accurately compared. This variation is not to be disregarded; in doubtful cases it may be of very great importance.

When aneurism exists in the arch of the aorta, it is more likely to be recognised by dulness on percussion than when the ascending portion of the vessel is the part affected. This arises from the lung towards the central line being thinner, and more easily displaced, or entirely pushed aside, than in the parts previously referred to.

Aneurism of the descending aorta, if of large size, may be attended with dulness on percussion on either side of the spine, though it more commonly extends to the left side. It may project directly forward, and, by pushing aside the lungs, be the cause of increased dulness in the præcordial region.

Abscesses of the anterior mediastinum are accompanied with dulness on percussion of the particular part which they affect; and enlargement of the bronchial glands is said to be recognisable by dulness of the interscapular regions. This latter fact I have not yet been able to verify, and I cannot but think that the enlargement must be very con- 
siderable to produce appreciable dulness on the interscapular region.

The diseases, then, in which Percussion may afford useful information, are Bronchitis, Pneumonia, Phthisis, Emphysema, and Malignant disease of the lung; Hydrothorax, Pleuritic effusion, Pneumothorax, and Malignant disease of the pleura; Enlargement or displacement of the heart; Pericardial effusion; Aneurism of the large vessels, and Abscesses or other diseases of the Mediastina.

Should the student have any difficulty in appreciating or eliciting the distinctions of sound and resistance afforded by percussion, as pointed out in the preceding pages, he may take a useful practical lesson upon his own cheek. Thus, let him place the middle finger of the left hand upon the right cheek while in contact with the teeth, and lap it with the fingers of the right hand; he will then find the sound quite dull, and the resistance firm; let him now distend the cheek slightly with air, and repeat the process, - the resonance will then be moderate, and the resistance slight; when it is about half distended, the resonance will be great, and the resistance slight; and when rendered quite tense, the resonance will be diminished, and, as it were veiled, and the resistance will be found to be increased, but resilient. 


\section{CHAPTER V.}

AUSCULTATION, OR EXAMINATION BY THE EAR.

GrEAT as have been the advantages which have accrued from the modes of physical investigation which have beèn already, and which are still to be, noticed, it is to Auscultation more especially, combined with the study of morbid anatomy, that is attributable the vast progress which has been made during the present century in discriminating diseases of the chest. It is to Auscultation that the profession is mainly indebted for that measure of light which has supervened upon comparative obscurity.

By Auscultation, as regards the pathology of the chest, is meant the practice of listening to the sounds arising from the exercise of the functions of respiration and circulation, and of comparing those emanating from diseased organs with those ascertained, by previous experience, to exist in the healthy condition. To this may be properly added the explanation of the difference of the sounds occurring in disease, by the physical condition of the organs produced thereby. 
Auscultation, like percussion, may be immediate or mediate. It is immediate, when the unguarded ear is placed upon the chest, either bare, or protected with only a slight covering, and when, therefore, the vibrations of the elastic parietes are communicated directly to the organ of hearing. It is mediate, when between the ear and parietes is interposed a body which conducts the vibrations of one to the other. The opinions originally entertained of the great advantages of the stethoscope are now acknowledged to have been erroneous. Some auscultators prefer mediate, others immediate, auscultation. Each mode possesses some advantages, and each is liable, in certain circumstances, to some objections.

\section{Immediate Auscultation.}

The ear, when this mode is employed, is applied to, and firmly pressed upon, the different regions of the chest in succession, either quite bare or only slightly covered, in such a way that the organ may, as much as possible, become one with, and therefore participate in the vibrations of, the solid parietes upon which it is placed. This is in many respects a desirable mode of auscultating. It requires, or appears to require, less abstraction; it is more easily acquired; it causes no pain and little inconvenience to the patient by the pressure employed, which, in some instances, almost necessarily arise from the 
use of the stethoscope; and it is, usually, at least as effective as mediate auscultation, where it is applicable and when large surfaces are implicated by disease. Not unfrequently, indeed, sounds which are indistinct and appear indefinite through the cylinder, become sufficiently obvious and determinate upon the application of the ear.

But immediate auscultation is open to several objections. The rustling of the clothes of the patient, in consequence of a larger surface being covered by the side of the head than by the end of the stethoscope, is more likely to interfere with the sound proceeding from the chest. The hair of the auscultator himself, by the motion of the patient's body, frequently gives rise to sounds nearly resembling those arising from disease. These may be incorrectly supposed to proceed from the chest, and may thus become a source of error.

It is sometimes important, as an aid to diagnosis, to be able to define the exact situation in which a particular sound is heard with the greatest intensity or distinctness; as in affections of the valves of the heart, the aorta, \&c. By the unguarded ear we cannot effect this so perfectly, we certainly do not possess the power of localizing particular sounds so accurately, as by the aid of the stethoscope.

By some persons it may be considered indelicate to apply the naked ear, not merely to the bare chest, but even to the unrobed, though slightly co- 
vered, parietes of female patients. In other persons, motives of a different nature will incline the auscultator himself to avoid contact, or a too near approach to the integuments of his patient. In the one case, modesty, or prudery, may forbid the employment of immediate auscultation; in the other, uncleanliness may render it undesirable. Independently, however, of the real or presumed indelicacy of applying the ear in the case of female patients, it is often quite impracticable to do so, with good effect, in persons with largely developed mammary glands. There are also, it must be recollected, some situations in which the ear cannot be fairly and efficiently employed; as over the larynx and trachea, in the space above the clavicle, in the upper part of the axilla, and, in very thin persons, immediately below the clavicle, and in the supra-spinous fossa of the scapula.

Immediate auscultation, however, notwithstanding what has been said, may be employed with the best success in many cases, may be usefully applied for the confirmation of opinions derived from other sources, or the verification of sounds doubtfully or indistinctly heard by means of the stethoscope.

\section{Cautions respecting immediate Auscultation.}

These have been, for the most part, already referred to in the preceding observations; but it may be desirable that they should be more distinctly and separately stated. 
First, then, it is necessary that, in the case of female patients, the appearance of the slightest approach to indelicacy should be avoided. If, therefore, for the purpose of confirming a doubtful opinion, or for any other reason, it be thought desirable to apply the ear itself to the anterior part of the chest, the skin should be covered with some soft yielding material, either appertaining to the patient's dress, or obtained from the attendants. A soft towel, an old dinner napkin, or even a silk handkerchief, may answer the purpose perfectly well; though cotton should generally be preferred to articles of linen, wool, or silk, as these not unfrequently give rise to some rustling or crackling sound, when tumbled or pressed, which may interfere, or be confounded, with those emanating from within the chest.

In this mode of auscultation, it is most assuredly the solid parts of the ear, and the bones of the head, which transmit the vibrations of the solid parietes of the chest to the auditory nerve. The protection referred to as a covering is, therefore, the more necessary; because, as has been before stated, the entire ear should be evenly and firmly pressed upon the parietes of the patient, so as to cause the two to become, as much as possible, one vibrating body. The napkin or towel, employed as a covering to the chest, should be smoothly spread, and tightly drawn, over the surface, and 
all other garments should be so far removed, as not to come in contact with the auscultator's ear or head; extraneous sounds will otherwise assuredly arise, which may not improbably mislead him.

The examiner should carefully put aside his hair from around his ear, and, as far as may be, from the side of the head, in contact with the chest; since if any portion intervene between the ear and the chest, or come in loose contact with the patient's dress, a crackling or rustling noise will be produced, which may be easily mistaken for sounds proceeding from within.

It can be hardly necessary to state, that in the case of uncleanly individuals, male or female, some protection should be used, lest the examiner should derive from his patient more than he would desire to retain.

From what has been already mentioned, it may be scarcely requisite to remind the auscultator, that when the chest of male patients is thickly clothed with hair, the hair must be removed before immediate auscultation can be satisfactorily performed. In females with large pendulous mammæ, the gland must be drawn aside, and the skin rendered as tight and firm as it may be, without serious inconvenience to the patient: her own hand, where it can be employed, or some article of her dress, is the best instrument for this purpose. 


\section{Mediate Auscultation.}

The principles of mediate auscultation appear not to be always understood, even by many who constantly employ it. The stethoscope, of whatever form, or however composed, is simply a conductor between the parietes of the chest and the ear. The vibrations of those parietes are caused, or are transmitted, by certain sonorous or resonating substances within the cavity. Whether these vibrations originate in air, or in blood,whether they are conveyed by solid or semi-solid bodies, by fluids or by gas, they all ultimately reach the solid walls of the chest. It is by the vibrations of those solid walls that they ultimately reach the surface; and by the vibrations of those solid walls that they are all mediately or immediately conducted to the ear. The stethoscope is merrely a conductor. It may, indeed, as in the flexible stethoscope, conduct by the air contained in the hollow of the tube alone; it may, as in the ordinary stethoscope supplied with a nipple-like appendage for the external meatus, conduct both by air within the bore of the cylinder, and by its solid walls,-or it may, as in the solid stethoscope, and often in the tubular stethoscope with a flat earpiece, conduct by the solid walls alone. It does not itself increase, it does not intensify the sound; and though by it, sometimes in consequence of pres- 
sure locally applied, or other causes, a sound may be assuredly heard more distinctly through it than by the naked ear, it is still a simple conductor between the parietes and the ear.

How, then, should this conductor be made? What form of stethoscope is the best for general use? To this it may be generally replied,-That with which each individual can hear best. In this respect, fancy and prejudice may effect something; habit much more. But to answer the question satisfactorily, it should be, in the first place, recollected that solids are better conductors of sounds than either fluids or gases; and secondly, that the vibrations of sound are considerably modified, and decreased in intensity, by transmission from one medium to another; that however originating, and by whatever elastic substance they may be transmitted, the waves constituting sound are greatly altered, and by partial reflection and interruption, are decreased in number and extent, upon their entrance into a medium of a different kind. The waves of sound, therefore, become less powerful by being transmitted from solids to gases, or to fluids, as well as from either these to solids. As, therefore, solids are in themselves better conductors than either fluids or gases; and as the solid parietes of the chest (confining attention to that cavity) are the medium, by the vibrations of which the sounds of the thoracic organs reach the surface; 
it appears sufficiently evident that, upon theoretical grounds at least, the solid stethoscope is the best that can be employed.

I believe that, generally speaking, what is rendered probable upon theoretical grounds will be verified by experience; and that in the vast majority of cases the solid stethoscope will be found the most useful instrument in practice. I call the ordinary wooden stethoscope solid, because, from long experience, and from numerous and diversified experiments, it appears to me quite evident that it is by the solid walls of the stethoscope that sound is in all cases principally, and in many cases entirely, conducted.

It is not necessary, nor do I think it at all incumbent upon me, to point out the advantages, or what I conceive to be the disadvantages, of any particular form of stethoscope ; but it may be desirable that I should mention that particular form which long practice, as well as theory, has induced me to regard as the best.

Though, then, it is desirable that each person should make use of that stethoscope which, by experience, he finds best suited to his ear, or with which, from habit, he can hear best; yet I may observe, that as from repeated trials I have long convinced myself that, in the wooden instrument, the sound is principally conveyed through the wood itself, that, in fact, the solid is the chief, and 
in a great majority of instances, the sole conductor, the more simply that wooden instrument is constructed, the better will be its conducting power. I do not intend hereby to deny that with some persons, particularly those who employ a nipple to the ear-piece of their stethoscope, or the earpiece of whose stethoscope is much hollowed so as to allow of the existence of a considerable volume of air between the cylinder and the ear, some vibrations of sound are conducted by the air in the bore of the cylinder, directly to the membrana tympani; but I believe that in all cases the greatest effect, by far, is communicated by the wood itself to the solid parts of the ear, and from them to the membrana tympani and the bones of the head; and $I$ am convinced that this is the sole mode by which many, and myself in particular, appreciate sounds in auscultation.

If the external meatus be closed with lint, cotton, wool, or chewed paper, the sounds are almost equally audible. If a perfectly solid cylinder be employed, or the bore of a hollow instrument be stuffed with any soft substance, the effect is scarcely perceptibly decreased. That it is at all decreased, in however trifling a degree, is dependent, I imagine, in the one instance, upon the mass of the solid being greater, and therefore requiring more force to produce an effect between its molecules, equivalent to that created in a mass of less extent; 
and in the other instance, upon the fact that the substance introduced into the hollow of the cylinder, or of the meatus; interferes, in some slight degree, by acting as a damper, with the vibrations of the solids themselves.

What, then, it may be asked, is the professed object, and in what consists the real utility, of the bore of the solid stethoscope? The professed object is, that the vibrations of sound may be transmitted through the air in the tube. Its real utility depends upon its making the instrument lighter, upon its probably facilitating the vibrations of the solid, and, in some cases, particularly in those instruments in which a nipple is appended to the ear-piece, or the ear-piece is much hollowed, upon its contributing to the effect of such vibrations by the addition of those which are communicated by the air occupying the hollow space.

The distinctions sometimes made between the sounds of the breathing, of the voice, and of the heart, may, I think, be practically disregarded in deciding upon the form of the stethoscope to be employed. Each and all of these sounds are communicated to the surface through the solid parietes, and by a solid they are all best conducted to the ear. At such conclusions we should arrive from purely theoretical considerations, and though, as regairds auscultation, experiment and theory do not always appear exactly to coincide; though in 
some rare instances, from our ignorance of all the circumstances of the case, it seems to be difficult to explain the phenomena upon common acoustic principles; yet in this case, as regards myself at least, practical experience exactly tallies with the theory.

The stethoscope, then, which appears to be most convenient and advantageous for general use, is made of one material, without joints or additions of any kind. The material (cedar) of which it is usually made, is probably as good as any other. It should be from four to six inches long, with a clean, polished, quarter-inch bore. The shape may be according to the student's fancy; but it is desirable, for the sake of lightness, to have it somewhat hollowed in the stem. The rim of the bellshaped or thoracic extremity should be broad, and well rounded; so that it may be firmly pressed upon the parietes without causing pain, or much inconvenience to the person examined. The earpiece should be broad, so as to embrace the entire ear, and nearly flat, or so little hollowed as not to admit the presence of air between the wood and the ear. The wood itself should be rather dense, and the instrument stout in every part, that it may not be chipped or broken by every accidental fall.

Such is the stethoscope which, after trial of several kinds, appears to me to be the best suited 


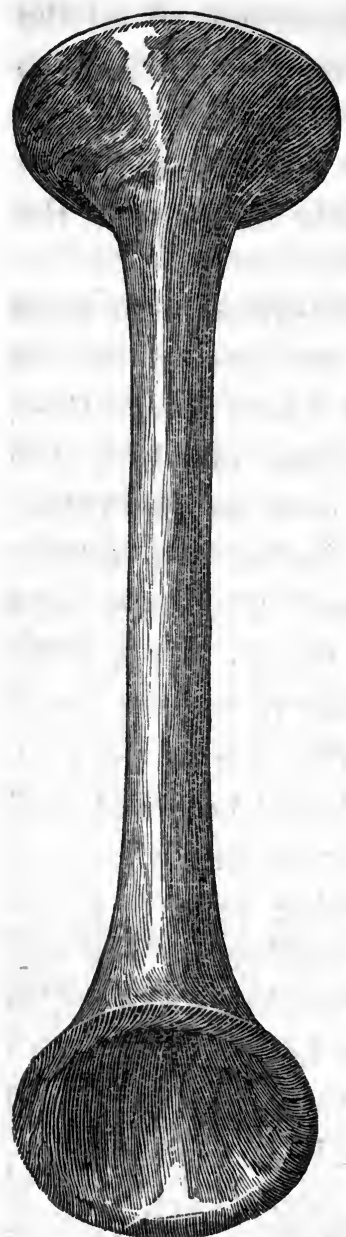

for practical purposes, and which I have for many years employed. A sketch of an exceedingly good instrument, formerly in my possession, which, although it may be fairly chargeable with being somewhat "out of drawing," gives, if viewed from below, a very tolerable notion of the form, may perhaps be here introduced with advantage, lest the directions above given should not be sufficiently explicit. It has been reduced one-third.

It may, however, be acknowledged that an auscultator can often hear better with a bad instrument, to which he has been long accustomed, than with a stethoscope of superior form and construction, with the use of which habit has not rendered him familiar. It may be indeed repeated, that the form of the instrument is of very trivial importance, and that the best stethoscope for any individual is clearly that with which he can hear best. 
There are, indeed, some cases in which the flexible stethoscope may be employed with advantage, even by those who are accustomed to use the wooden cylinder. But they are cases only in which it is inconvenient or impracticable to apply the solid tube; those, namely, in which it is important to examine the posterior parts of the chest, but in which it would be unsafe or imprudent to raise the patient, and he is himself unable to turn, or incapable of being turned, for the purpose. In such cases, the infundibular end of the instrument may be passed under the body, without seriously disturbing the invalid, and important information may be occasionally acquired thereby, more especially by those who are accustomed to the use of such an instrument.

\section{Mode of using the Stethoscope.}

The instrument, grasped in the centre by the two fingers and thumb of the right hand, as a pen, should be first flatly placed, and firmly pressed, upon the parietes of the chest. The cylinder must always be at right angles with the surface to which it is applied, and the head of the examiner must be always bent to the extremity of the instrument, and the instrument not made to incline to his head. Thus, if the patient be upon his back, and the front of the chest be the part under examination, the 
stethoscope must be perpendicular, or nearly so; if the space between the front and the sides of the chest be submitted to investigation, it must be placed obliquely; and if the side of the chest be the region explored, it must project horizontally therefrom, and the head of the examiner must be depressed or elevated accordingly. If this be not attended to,-if the entire rim of the stethoscope be not firmly pressed upon, and be in perfect contact with the parietes, little will be heard, because few vibrations will be communicated to the instrument. I dwell particularly upon this fact, because I have often observed inexperienced students apply the instrument imperfectly, and assert that they could not hear what was obvious to other observers. The ear should be evenly adjusted upon the broad, nearly flat end of the instrument, and the pressure of the head be so firm as to make the parietes of the chest, the conducting medium (the stethoscope,) and the ear, one connected vibrating body. This cannot be effected without considerable pressure, which, however, should not be so great as to cause pain to the patient. To prevent this, not unfrequent, result of the application of the stethoscope, the edge of the funnel-shaped extremity of the instrument should, as before stated, be broad, and smoothly rounded off. The contact of the fingers with the instrument is then to be removed, and observations made; first as regards 
the breathing, and then voice, and the cough, as will be further explained in a future page.

The undivided attention of the examiner must be directed to the sounds passing through the tube; his senses should be dead to all besides. He must hear nothing with the other ear, the abstraction must be perfect. Generally speaking, this capability of abstraction is to be attained only by habit; but if noises in the chamber, the ward or the adjoining room, or street, interfere with the examiner's power of discriminating the sounds proceeding from the chest, he may place a finger of the disengaged hand over the meatus of the unemployed ear, and thus exclude all other sounds. He may by the very act contribute to the abstracted attention, which, in many cases, is absolutely requisite to arrive at satisfactory conclusions. It has been said that the stethoscope should be grasped by the fingers of the right hand, when, of course, the right ear would be employed; but it will conduce greatly to the student's aptitude in exploration to be able to employ both hands and both ears with equal facility.

\section{Cautions respecting mediate Auscultation.}

It may be well to repeat, in the form of cautions, some particulars which have been already mentioned in the preceding directions. Let, then, the examiner be especially careful that the entire circle 
of the thoracic end of the stethoscope come in close contact with the parietes. If, from unequal pressure, improper position of the patient, or oblique direction of the instrument, the contact be not entire, the vibrations of the walls of the chest will be very imperfectly transmitted.

In some cases, as in persons naturally very thin, or greatly reduced by exhausting diseases, the depressions existing between the ribs in some regions of the chest, and those frequently the most necessary to be minutely examined, are so great as to render it impossible that the whole circle of the stethoscope can come in contact with the skin. The intercostal spaces are left untouched, while the skin over the ribs is submitted to severe pressure. Under such, and all similar circumstances, little, or nothing, can be heard through the stethoscope.

Two expedients may be then adopted: the examiner may either make a pad of soft linen, or lint, by which the depressions between the ribs may be filled up, and the skin over the ribs protected; or he may adopt the other, and usually the preferable expedient, of discarding the stethoscope altogether, and using the unprotected ear. The latter plan is the more desirable of the two, as the pad necessarily in some measure interferes with the facility with which the sounds are transmitted to the ear. The naked ear should therefore be in such cases adopted, excepting in those parts of the chest to which it cannot be fairly applied. 
The stethoscope must be carefully prevented coming in contact with the patient's garments, with the bed-clothes, with his extremities, or with any other part of his body than the chest itself. This caution is even more necessary in mediate than in immediate auscultation. The slightest friction of the garments, or of the hand, is so perfectly conducted by the instrument, as most materially to interfere with the distinctness of, if it does not overpower, the sounds proceeding from the chest.

In very stout persons the pressure of the instrument must be increased, so that the adipose tissue may be compressed, and, as far as possible, rendered identical, as regards conducting power, with the bony parietes which it covers, and the free vibrations of which it in some measure prevents. In persons whose integuments are oedematous, the pressure of the instrument must be equally great, or even greater, than in fat persons; so that the serum may be entirely squeezed out of the cells immediately below the stethoscope. Loose fat and diffused serum are bad conductors of sound, when compared with solids. Hence the importance of the caution just delivered.

In reference to œdema, however, another caution is required. This arises from the frequently observed fact, that the escape of serum from the cellular membrane by the pressure of the instrument itself, gives rise to a sound very similar to one 11* 
frequently proceeding from within the chest. The sound referred to is pleuritic rubbing, one form of which, possessing a jerking, or interrupted character, the noise produced by the escape of the serum from the cells under the pressure of the stethoscope, or, it may be, by the slipping of the instrument over the skin, in consequence of such escape, so exactly resembles, that if the patient continued to breathe during the whole of the examination, the two sounds would be distinguished with the greatest difficulty, if, indeed, they could be at all distinguished from each other. An obvious distinction of course exists in the fact, that the rubbing of the pleura is only present during respiration; while the odematous creak, so long as any serum remains to be squeezed out of the cells, persists though the breathing be temporarily suspended. The sound, however, so precisely represents certain forms of the rubbing existing in disease of the pleura, that I have, in the presence of pupils, occasionally illustrated the latter sound by pressing the stethoscope upon an oedematous thigh.

If, indeed, the oedema be soft, and the integuments flabby, the peculiar sound to which I have referred is not always produced by pressure, or it is of a much less sharp and decided character than when the swelling is firm and tense. On these two accounts, then,-first, because the infiltration of the integuments obstructs the sounds proceeding 
from the chest; and, secondly, because on pressure it gives rise to another sound, which may be easily confounded with one of intra-thoracic origin; the serum of oedema should be carefully pressed out of the cellular membrane before auscultation is attempted.

5. Combined use of mediate and immediate Auscultation.

From what has been already said, it will be evident that, on some occasions, and in some parts, the ear, and on others the stethoscope, may be most advantageously employed. As, therefore, it will conduce to the efficiency of his investigations, it is desirable that the student should be practically conversant with both modes of auscultating. Let him on no account neglect the use of the stethoscope, because he finds that, under ordinary circumstances, he can hear better with the unguarded ear; as in practice cases will constantly occur to him in which he will be compelled to employ the cylinder, or to leave certain parts of the chest unexamined.

The plan of proceeding which will perhaps be found on the whole most useful, is in each case to practise both mediate and immediate auscultation. Thus, on the anterior part of the chest, and particularly the acromial, the axillary, and the infraclavicular regions, the stethoscope may be used; while in the lateral regions, and all the posterior 
surface of the chest, excepting the space above the spine of the scapula, the ear will be found at least as effective, when unguarded, as when supplied with the solid conductor. If the patient be very fat, or the integuments be infiltrated with serum, mediate is preferable to immediate auscultation; as with the stethoscope more direct and local pressure can be made than with the ear, which, though not so far removed, in such cases comes not into such near acoustic relation with the solid parietes, as when the solid conductor intervenes between them.

In all examinations of the larynx and trachea, it is almost always necessary to employ the stethoscope. In all affections of the heart excepting when great tenderness exists upon pressure, the stethoscope is to be preferred to the ear; while in the auscultation supplementary to succussion, to be afterwards noticed, and in the superficial examinations made through stays and petticoats, the ear, if it be evenly placed, and the patient's body be firmly pressed against it by the hand placed upon the opposite side, will be ordinarily a more effective auscultatory instrument than the cylinder.

\section{Auscultation of the Organs of Respiration.}

It is absolutely necessary, as has been before stated, that the student be perfectly familiar with the sounds naturally existing in the chest, previously to his becoming acquainted with, or being 
capable of judging of, those which are abnormal; just as he must be conversant with the healthy appearance of organs before he can fairly expect to become a morbid anatomist. The normal and the abnormal sounds, or sounds of the respiration and the voice, therefore, will be the first noticed, and subsequently those which exist in disease.

\section{Of the Natural Respiration.}

To enable the examiner to judge correctly of the healthy or morbid condition of the respiration, ordinary inspiration is scarcely sufficient. It is desirable that the air should be more generally diffused through, and should more perfectly inflate, the pulmonary cells, than it does in common inspiration. In nice examinations, therefore, the patient should be desired to fill his chest by a succession of forced inspirations. The examiner will be thereby enabled to make his observations upon the character and duration of the expiration, as well as upon those of inspiration; the former of which, in ordinary breathing, is sometimes scarcely audible, and differs from the latter in some other important particulars, which will be afterwards referred to. To prevent confusion, it may be well to confine attention, in the first instance, to the inspiration. [Simple as the process may appear, it is by no means an easy matter to make some per- 
sons take a really deep inspiration, in such a way as to enable the auscultator to judge of the condition of the lung. Their awkwardness is sometimes so great as really to have the appearance of design. First, they raise their shoulders without inspiring at all; then they expire deeply; then they draw in the air through the compressed lips, or constricted fauces, in such a manner as to give rise to noises which overpower the sounds originating within the chest; afterwards they protrude the abdominal muscles, forcibly raise the eyelids, puff out the cheeks, and then perhaps go through the entire series of awkward efforts again. To tell them to take a long breath, to inspire deeply, to heave a sigh, to fill the chest, to draw in the air, to raise the ribs while they breathe, or even to show them how to do so, is of no avail. The more you talk, and the more they appear to try to effect the object you desire, the farther do they remove from that object; the more stupid in this respect do they seem to be. But they often involuntarily do exactly that which, after many and diversified attempts, they have been unable to effect. Thus, if told to cough, and continue coughing for a short time, they will, indeed they must, from the mere exhaustion caused by the repeated short expirations, which constitute that act, spontaneously take such a soft, quiet, yet deep inspiration, as all previous efforts had failed in causing them to accomplish.] 
If the ear be applied to the stethoscope, evenly placed upon the larynx or trachea, and gentle pressure be made, the sound caused by the ingress of air during the act of respiration will be observed to be hoarse and hollow, like that produced by blowing, while the lips considerably compressed, through a pipe of large calibre, as through the bore of the stethoscope; or that which is sometimes produced by a draught of air through the key-hole of a door. It is called tracheal respiration. When a similar sound is heard, as the result of disease, in other parts of the chest, the breathing is said to be tracheal, or, more commonly -for the distinction between the two is only one of degree-bronchial or tubular.

If, instead of being placed upon the neck, the stethoscope be now put upon the upper bone of the sternum, below the sterno-clavicular articulation, or between the scapulæ upon either side, and the individual be desired to breathe deeply; a sound very much like, but not exactly similar to, the tracheal respiration will be heard by the auscultator. The sound may be generally, but, it must be acknowledged, by no means universally, observed in the state of health in the situations indicated. It is sometimes absent in all these localities; but occasionally, when inaudible on the front of the chest, it may still be heard close to the spine, at a level with the centre of the scapulæ. It is not so 
loud, or so hollow, as the tracheal sound. It may be represented as its diminutive. It resembles the noise occasioned by blowing through a small reed, or a quill of moderate size. It arises from the friction caused by the rush of air through the larger bronchial tubes, and, wherever heard, is therefore called bronchial respiration, or tubular breathing. If discovered in other situations than the upper part of the sternum, the inner side of the infra-clavicular, or the inter-scapular regions, it may with tolerable certainty be regarded as morbid; though it may be occasionally heard less distinctly than in those regions in the axillary spaces; particularly that of the right side.

It may be here observed, that the natural breathing not only presents varieties, as to tone and distinctness, in different individuals; but also that similar regions, upon opposite sides of the same person, may slightly vary in these respects, as in some others, quite independently of disease.

When the stethoscope is placed upon most other parts of the chest than those previously specified, a sound is heard by the auscultator during inspiration very different from those which have been mentioned before. It is perhaps difficult to define its character correctly. It is soft, scarcely sonorous, equable, breezy, and, during inspiration, continuous. It may be pretty accurately copied by 
gently drawing the air through the partially compressed lips; it may be compared to the song of a gentle gale in the thick summer foliage; or to the whisper of the retiring wave upon a sandy coast. It is called the respiratory or vesicular murmur, or, by some authors, the "breath sound," on account of its being the characteristic of healthy respiration, and of its being clearly connected with the vesicular tissue of the lung. It probably depends upon the friction caused by the admission of the air into the irregular cavity, and its passage over the uneven surface of the pulmonary vesicles.

It varies considerably in intensity in different regions of the chest. It is most distinct in the acromial, the central and lower part of the superior sternal, the infra-clavicular, the axillary, and the subscapular regions. It is less distinct in the lateral, the right mammary, the scapular, and the interscapular regions; still less in the hypochondriac; and least of all in the inferior sternal and the inner part of the left mammary region.

Independently of the variation of the intensity of the sound in the different regions of the chest, whether the variation arise from the position of the organs, the amount of pulmonary tissue beneath the ear, or the facility or difficulty with which the inspired air reaches the pulmonary cells, the two sides of the chest frequently vary a little in respect 
to the loudness of the respiratory murmur. Thus it is, usually, rather louder in the acromial, scapular, and infra-clavicular regions of the right side, than in the corresponding regions of the left side; but in so slight a degree, as to be scarcely worthy of consideration, in a practical point of view.

The respiratory murmur may, both locally and generally, be more loud, or less distinct than natural, in persons who are quite free from any appreciable disease. It may also be harsh, or rough, scarcely audible, or altogether absent.

Thus, in childhood, and in youth, the respiratory murmur is more loud than in adult life, and especially than in old age. This probably arises from the denser nature of the tissues, and the greater functional activity of the lungs, in the young than in persons advanced in life. From this circumstance, it is called, whether existing in children, in youth, or, from whatever cause arising, in age, puerile or supplementary respiration. Puerile, because it is the normal state of the respiration in children; and supplementary, because when one lung, or part of a lung is disabled, it supplies, by the increased activity of the other lung, or of another part of the same lung, the defective action of the diseased organ or part.

Indeed, as it is frequently observed to exist in the healthy portion of a lung, or in an entire lung; when the remaining portion in the one case, or the 
opposite lung in the other, is greatly embarrassed, and prevented performing its accustomed function; and as this healthy portion, or this sound lung, seems under such circumstances to have its functional activity increased, and thus to supply the deficiency of the diseased parts, it is with great propriety called supplementary respiration. It is always heard when the healthy respiration is more than ordinarily active, as in persons "out of breath," as it is called, from strong exertion, as running, dancing, \&c., or after the respiration has been voluntarily suspended for a time, and the individual breathes quickly to restore the normal balance of the circulation through the pulmonary organs.

The respiratory murmur of some persons appears naturally to be harsh or rough, or to be so, at least, without any other evidence of pulmonary disease than is afforded by such harshness or roughness. The cause of this is not evident, though, from what is observed in disease, it seems not improbable that it is dependent upon some either congenital or acquired peculiarities in the smaller divisions of the bronchial tubes, rather than upon the vesicular tissue of the lung itself. It may be either local, or general. Its more cômmon seat (when local,) is the axillary space, in which it is far from unfrequent. If it be observed in other situations, and it be, at the same time, not heard over the 
whole of those regions in which the pure respiratory murmur is commonly heard most distinctly, it must be regarded at least with suspicion.

Harsh or rough breathing is, to the educated ear, sufficiently distinct from puerile respiration; though, to the inexperienced in auscultation, it may be difficult to convey a clear notion of what constitutes the distinction; and it may be still more difficult to make any but those possessed of a delicate sense of hearing appreciate the difference. It is, however, desirable at least, if not necessary, to make the attempt. In harsh respiration the character of the sound is changed or modified; in puerile respiration its power is simply increased. Though the former may exist in health, it is ordinarily indicative of disease, however slight: the latter is merely an evidence of increased activity in the function of respiration in the lungs, or in that portion of lung in which it is observed; whether that increase be dependent upon the tender age, upon the accidental rapidity of the circulation and respiration of the individual, or upon the defective action of some other portion of the pulmonary tissue, and consequent increased activity of the part itself. Puerile respiration is in fact a loud vesicular murmur, an exaggeration of the natural sound of respiration. It is as the "forte," to the "piano," of the same note on the same soft-toned instrument; while the harsh respiration, or rough 
breathing, is the "forte," of the same note, but upon a loose and jarring string; or, to carry on a former illustration, in the one case the same soft breeze passes through a greater number of trees, in the other the breeze is increased to a moderate gale through a plantation without leaves.

The strength of the respiratory murmur, instead of being increased, may be diminished; though no disease exist in the chest. This imperfection of the respiratory murmur is usually observed either in parts of the lungs which have been little used, as in the lower regions of the chest of females accustomed to tight lacing; or in persons suffering from deformity, whether congenital or acquired; or in the chests of persons considerably advanced in life. In each case it probably arises from defective respiratory function. In the two first mentioned cases the defect results from want of use, and consequent imperfect expansion, of the lung; in the last case it proceeds from atrophy, and consequent defective functional activity, of the pulmonary tissue. It may, indeed, be regarded as the natural character of the respiratory murmur in old people, and may therefore be called "senile," as that existing in childhood is termed "puerile," respiration. Occasionally the respiratory murmur is entirely absent from one, or a part of one lung, though no disease be present in the organ itself. This condition, however, probably never exists without either some 
mechanical obstruction to the ingress of air, either in the air tubes or upon the exterior of the organ. The more common causes of the absence of respiration in a portion of the lung, independently of disease in the pulmonary tissue itself, are the presence of foreign bodies in the bronchial tubes, ' or the compression of their calibre by aneurisms or other morbid growths. It may also arise from penetrating wounds of the chest, by which the lung itself remains uninjured, but in consequence of which it collapses from the admission of air into the pleura.

None of these conditions, however, nor any of those arising from malformation, or compression of the ribs by stays, can, properly speaking, be regarded as states of health, though in none is it necessary that the pulmonary tissue, or any of the respiratory organs, should be themselves actually diseased.

The preceding observations, as previously hinted, have especial reference to the murmur of expiration; as from this that of expiration differs in some important particulars. The difference consists in the decreased loudness and duration of the expiratory, as compared with the inspiratory sound. The tone and general character are the same in both cases-the one is merely louder and longer than the other; the difference is merely one of degree. 
An attempt has been made accurately to define this difference by figures. But as probably no two persons would exactly coincide, even in a given case, as to the purely relative proportions between the two, of which they possessed no means of determining accurately the admeasurement, the attempt was not likely to be successful, or generally applicable.

The difference has in the main, as I conceive, been much overstated. The proportion pronounced to exist in the healthy condition, by the proposer* of the plan for marking the difference by figures, was, in regard to intensity as well as to duration, as 10 to 2; that is to say, the sound of inspiration was considered to be naturally five times as long, and five times as loud, as that of expiration.

Now, though a marked difference does exist in the state of health,-and it is of great importance that this should be particularly noticed, in consequence of its bearing on disease, in which the proportions are changed, and sometimes even reversed, -yet it may be safely asserted that the disparity is not so great as that which has been stated by the proposer of the employment of figures for its admeasurement. Perhaps the proportion of 5 to 2 may be regarded as nearer the truth; though each individual examiner will probably form a

* The accomplished and really original Fournet. 
standard for himself on this, and on many other subjects which cannot be accurately measured, or strictly defined.

As to tone and softness, as has been before stated, the murmur of expiration naturally resembles that of inspiration. It is simply less loud, and less prolonged. If, therefore, its character be different; or if its intensity, and its duration, or either of them, be materially increased,-if, that is to say, they nearly approach, equal, or surpass those of inspiration,-the condition may be regarded as an abnormal one; and the cause may be almost universally attributed to structural alteration, and, if not occurring in a person in advanced life, to disease properly so called.

\section{Of the Voice.}

Some facts may, in disease, be occasionally elicited by auscultation of the Cough, in contradistinction to that of the Voice. But as the sound of each is connected with the same portion of the respiratory organ, as they are similarly conducted by the same parts, and as in health they produce very much the same effect upon the ear, when heard through the parietes of the chest, -as, moreover, cough can scarcely be properly regarded as existing in health, - the Voice and the Cough may, in the following observations, be spoken of together.

If the end of the stethoscope be placed upon the 
larynx of a healthy person, and, while one ear of the examiner is applied to the free end of the instrument, and the other ear is closed by a finger of the disengaged hand, the individual examined be directed to talk, his voice will pass into the stethoscope directly into the ear, and will appear as distinct as, and perhaps even more loud than, if his lips were close to the ear. This is perfect Pectoriloquy (chest talking,) and the best possible illustration of that important sign, as it exists in some forms of disease.

If now the end of the cylinder be removed from the larynx, and placed upon the lower part of the trachea, and the person be directed to talk as before, it will be noticed that, though the voice appear in some measure to permeate the tube, and though the individual still seems to speak into the ear, yet the voice is not so clear and distinct as in the former case. Such is imperfect Pectoriloquy.

Let the examiner now move the stethoscope to the upper bone of the sternum, to the cartilages of the second and third ribs, or to the interscapular regions, and again direct the person to talk. A fresh modification of the voice is now observed. It does not appear to permeate the instrument at all, and, though it may be loud, is comparatively distant and indistinct. The sound appears to be obstructed by the intervention of a bad conductor between 
the mouth of the speaker and the ear of the listener, or as if a veil was interposed. This is Bronchophony (bronchial sound.)

If, finally, the end of the stethoscope be placed upon other parts of the chest than those specified, the sound of the voice is still heard, and differences of intonation can be recognised, but under ordinary circumstances articulation can be scarcely said to exist. The distinctness, as well as the loudness of the sound, diminishes the farther the stethoscope, or the ear, (if that be now employed) is removed from the larynx, and from the larger bronchial tubes: the former is the source of the sound, and the latter are its best conductors. Thus it will be found that, at the most distant parts, as over the thin edge of the lungs, the voice, if heard at all, is reduced to an indistinct buzzing.

Such, then, are perfect pectoriloquy, imperfect pectoriloquy, bronchophony, and the distant voice in the natural condition; such are they also if well developed when they occur in disease. It must not, however, be supposed that there exists any defined line of demarkation between each, so that it can be said here ends pectoriloquy, and here begins bronchophony. They all gradually merge into each other; they are not distinct species, but merely varieties of sound. So that what one auscultator may call imperfect pectoriloquy, another may probably denominate bronchophony. But to 
deny the distinctions between well-marked pectoriloquy and bronchophony (I speak not of the indications afforded by them, but of the sounds themselves) appears to me tantamount, on the part of the recusant, to the acknowledgment that he has a bad ear, or is an indifferent auscultator.

Independently, however, of the differences of the resonance of the voice resulting from the distance of the source of sound, and from the intervention of a good or bad conductor, and irrespectively of the acknowledged fact, that perfect pectoriloquy gradually becomes imperfect, that this imperfect pectoriloquy gradually merges into bronchophony, and bronchophony into the ordinary state of the voice, as heard generally over the parietes of the chest, there are some varieties, in respect to the thoracic voice, which are worthy of attentive consideration. Thus it is very generally rather more distinct upon the right side, and particularly below the right clavicle and over the right scapula, than in similar situations upon the left side. This may, I believe, be regarded as the rule, though some exceptions occasionally occur. The voice is also much more resonant in some persons than in others. Thus a shrill or acute voice is generally more distinct and clear, though it be not more loud than one which is hoarse or grave. It is usually more audible in thin persons than in those whose integuments are loaded with fat, or 
infiltrated with serum, and, the power of the voice being equal, and both free from disease, it is more resonant in persons with contracted chests than in those in whom the thoracic cavity is largely developed. It is generally also more distinct, coeteris paribus, in females than in males; probably from the greater shrillness of the voice in the former than in the latter.

In some few persons indeed-most commonly females and youths-the voice is, in certain situations, as below the clavicles, or over the scapulæ, so clear, distinct, and loud, as to approach, if not to attain, the character not only of bronchophony but of pectoriloquy. Hence arises the necessity of the caution, in all examinations of the voice, always to compare the corresponding parts of opposite sides with each other. If after such comparison both sides be found equal; or nearly equal, in resonance, and if after minute examination no other physical signs of disease can be discovered, the unusual resonance may be ascribed with tolerable safety to natural peculiarity, and it may be assumed that the individual under examination has a natural bronchophony or pectoriloquy, as the case may be.

In consequence of some tones or syllables being better conducted than others (and it is remarkable that it is not always the same tones which in different persons possess this superiority,) it is desi- 
rable that the same words or syllables should be pronounced during the examination of every part and of each side. It is on this account that it is usual to desire the person under examination to repeat the numerals, one, two, three, four, \&c., as in them little or no variety is likely to occur.

\section{Auscultation of the Organs of Respiration in Disease.}

It will be found convenient, in examining the phenomena afforded by auscultation in disease, as in the healthy condition of the respiratory organs, first to confine attention to the abnormal sounds which accompany the breathing itself, and afterwards to consider the alterations which take place in the voice and cough.

\section{Of the Respiration in Disease.}

It cannot be too forcibly impressed upon the mind of the student, and it may therefore be here again brought to his recollection, that there are few, very few single signs, if indeed there be any, which are purely characteristic or pathognomonic of any particular disease. The more he observes for himself, and the more attentively he studies the products of disease, the more convinced will he become of this truth. Auscultation and its handmaid arts often enable us, with almost perfect certainty, to 
predict various morbid changes, but of the exact nature, or rather of the cause of those changes, they tell us little or nothing. Physical signs merely indicate certain physical conditions, which may be produced by two, or by a variety of causes. The physical condition of the organs may be very generally detected by auscultation, and the other different modes of exploration treated of in this work; but information in respect to the morbid process by which it has been produced must be sought for in other channels, and be determined by other means; as, by the general symptoms of disease, or by the history of the respective cases. Let the student ever recollect, and excuse me for repeating again and again the axiom which $I$ am so anxious to impress upon his mind, that physical signs are indicative only of certain physical conditions, and not of particular diseases.

The morbid sounds of the respiration may, to avoid confusion and perplexity, be conveniently first divided into dry and moist sounds. By dry sounds are intended those which arise from alteration of the calibre of the larynx, trachea, or bronchial tubes; from changes in the capacity, or from obstruction, of the pulmonary cells; or from roughening of the pleural surfaces. By moist sounds are meant those in which, either without, or in addition to, the alteration, obstruction, or roughening giving rise to the dry sounds, fluid secretion 
or effusion (whether of blood, or serum, or pus,) exists in the passages or cells, or in which air is mixed with fluid in the pleura.

2. Dry sounds in Diseases of the Air-passages and the Lungs.

(a.) Larynx and Trachea.-If, from inflammatory engorgement of the lining membrane, from solid effusion upon the internal surface, or from fluid effusion beneath it, or if, in consequence of preceding ulceration, any contraction exists in the rima glottidis, or in the course of the trachea, which gives rise to constriction of the tube, and thereby impedes the free ingress and egress of air, a wheezing, whistling, or crowing sound is produced by the act of respiration. It is loud or slight, according to the amount of the obstruction, and to the power of the patient, and is generally louder during the inspiration than during the expiration. Of this nature is the harsh or crowing respiration existing in croup, and the loud wheezing of oedema of the glottis. A foreign body fixed in the oesophagus, and pressing upon the trachea, or situated in the trunk of the air-passages itself, gives rise to similar sounds.

If a tumour,-as an abscess, an aneurism, or a morbid growth,- - exist in the vicinity, and exert such pressure upon the cartilaginous box or tube as to produce obstruction to the passage of air, the 
mechanical effects in either case are nearly the same; and the sounds which result from it, though they may vary a little in different cases, are also nearly the same. To the crowing convulsions or false croup of children, in which, though the cause is different, the mechanical effect, so far as regards the sound produced by respiration, is the same, and also to the laryngitis and the oedema glottidis of the adult, similar observations may be applied.

In each of these cases the noise is usually sufficiently obvious, and the evidence of obstruction is sufficiently clear, independently of auscultation. By the stethoscope we are enabled merely to say that obstruction exists, and to indicate its seat. But the nature of that obstruction it telleth not. We may, indeed, be able to state that, in the case of a foreign body, the cause of obstruction is fixed or moveable-that it is now in one part and now in another-but the feelings of the patient, if he be conscious, are usually amply sufficient to assure us of this.

Is, then, auscultation useless in diseases of, or in accidents occurring to, the larynx and trachea? It certainly is not. It is true that it merely indicates that obstruction exists, and does not acquaint us with the nature of that obstruction; that by it we are generally enabled to effect that which is often of the utmost importance-viz. to point out the exact seat of the obstruction. Without the stethoscope it may be doubtful whether the impedi- 
ment to the breathing is situated in the larynx or in the trachea, or whether it is high up or low down in this tube, as the sounds heard by the ear are very similar, if not identical in either case. But by gently placing the end of the stethoscope upon the larynx, and carefully tracing along the respiratory tube, and attentively listening to the sound of the breathing as we pass it onwards, we are usually enabled, pointing to a particular spot, to say with tolerable precision, "There is the seat of the obstruction."

This capability of localizing the source of obstruction is the only benefit afforded by auscultation in diseases of the larynx and trachea, properly so called, with which I am particularly acquainted.

But though single, it is of immense importance, and may be the means of restoring life when death is impending. It need scarcely be said, that when obvious tumours are pressing upon the trachea or larynx, we may be enabled by means of auscultation to state that such tumours are, or are not, aneurismal.

When, instead of being constricted, the narrow opening of the larynx is widened; if, instead of being thickened or contracted, the vocal cords be considerably ulcerated or destroyed, the natural reed-like sound which exists in the healthy respiration disappears, and a mere blowing noise supplies its place, similar to that produced by blowing 
through a reed of large calibre, without more compression of the lips than is necessary to support the tube, or through the hollow of the stethoscope, with the mouth open sufficiently to present no obstruction to the forcibly expired air.

(b.) Bronchial tubes.-It has been already stated that the sound of the air passing through the bronchial tubes in the natural condition is, in most parts of the chest, inaudible; and that, under ordinary circumstances, it can only be heard over and near to the upper part of the sternum, and especially at the root of the lung in the interscapular space, where the larger tubes are near to the surface, and little or no pulmonary tissue intervenes between them. Sometimes it is scarcely audible even there. This probably arises from the respiratory murmur contributing to obscure the bronchial sound, which is but feebly conducted by the soft spongy tissue of the lung itself. It will be seen as we proceed that the case is altogether altered when the vesicular murmur is absent, and when the lung, from consolidation, or other causes, becomes a good conductor of sound.

When the larger bronchial tubes are in any way contracted, whether by pressure upon their exterior, from deposit upon their interior, or from thickening of any of the structures of which they are composed, the transit of the air through them is of course impeded. The obstruction thus pro- 
duced gives rise to sounds which vary considerably according to the nature and extent of the obstructing cause, and the quality of the conducting medium existing between the tubes and the surface of the chest.

Thus, when the mucous membrane of the large tubes is merely thickened from increased vascular supply, and when they are free from morbid secretion, and are not dilated, as in the very early stage of bronchitis, or in the quiescent state of the membrane of those persons who habitually suffer, after slight exposure, from a chronic form of the malady; or when the walls of the tubes are themselves thickened from long-standing inflammatory change, or "hyper-nutrition," a loud, rough, deep, snoring noise will generally be heard upon the application of the ear to the chest, which clearly depends upon the vibration of the tubes resulting from their narrowing, and the consequent impediment they afford to the free passage of the air. This is the ordinary form of sonorous rattle or rhoncus ("râle sonore.")

When, together with this general constriction, there exists enlargement of the tubes in certain parts,-when, that is to say, partial dilatation is combined with general thickening and contraction, then, in .addition to the loud snoring noise, there is often a deeper sound, a sort of puffing or blowing, which sometimes approaches the character of the 
sound produced by blowing into a wide-mouthed bottle. If the dilatation be very great, so as to produce, as it sometimes does, a cavity of considerable size, the same sound may be heard even though no constriction exist at other parts. The physical conditions necessary for its production are the passage of air through a tube or through a moderately-sized opening into a free space much larger than the opening itself.

When, instead of the larger, the smaller tubes are the parts principally constricted, the sound is of a higher pitch; hissing or squeaking noises are then heard, which constitute the sibilating rattle or rhoncus ("râle sibilant") of authors.

The sonorous rattle then depends upon some obstruction, produced either by compression or thickening, absolute or comparative, of the larger bronchial tubes, while the sibilating or hissing rattle, or rhoncus, generally arises from a similar affection of the smaller tubes. It is to the sonorous rattle or rhoncus what the fife or the kit is to the flute or the violoncello. These rattles may exist either singly or together, and independently of the presence of fluid secretion in the bronchial tubes, and consequently without any moist rattles or rhonci, though they are most commonly conjoined with the latter sounds.

Together with this obstruction of the larger or smaller bronchial tubes, and very commonly as 
its direct mechanical effect, when it has been long continued or frequently repeated, there exists a dilated condition of the pulmonary vesicles, and consequent thinning, or it may be even rupture, of the delicate membrane which separates them from each other. This condition of the pulmonary tissue constitutes emphysema of the lungs. As these chronic affections of the tubes and of the aircells are commonly associated together, as cause and effect, it may be well to consider them in immediate succession.

When the air-cells are considerably dilated, and their partitions, or the tissue intervening between them, are consequently reduced in thickness, though the bulk of the lung, or of the part of the lung affected, is increased, the amount of pulmonary tissue in any given portion of the diseased organ is decreased. The natural elasticity of the tissue is diminished, and in extreme cases is destroyed. The area of the cells is not only absolutely greater than in health, but, comparatively with the calibre of the tubes, it is also enlarged. The lungs, when emphysema is general and extensive, are in a state of more than forced inspiration. They are too full of air, and incapable of expelling it. The necessity of fresh air begets a constant effort to draw still more into the chest by the extraordinary efforts, and forced contractions of the muscles of inspiration, while expiration is but feebly assisted by the mus- 
cles, which, under certain circumstances, contribute to that act. Expiration, in fact, unaided, or only slightly aided, by the elasticity of the pulmonary tissue, and assisted in only a trifling degree by the abdominal muscles, is principally, or almost solely, effected by the elasticity of the ribs. The necessary results, as regards auscultation, of this disease of the lung, will then be readily understood to be -1st. That the act of inspiration is shortened, in consequence of the quick and strong effort to effect it. $2 \mathrm{nd}$. That the natural inspiratory murmur is greatly decreased, both as regards intensity and duration, in consequence of diminished density of the vesicular tissue of the lung on the one hand, and of the small amount of air inhaled on the other. 3rd. That the duration of the expiration is greatly prolonged by reason of the deficiency or absence of elasticity in the pulmonary tissue; and 4 th, that a loud wheezing noise accompanies the expiration, vastly exaggerating the natural sound, the result of the obstruction which exists to the exit of the air through bronchial tubes comparatively or absolutely contracted.

The genuine auscultatory signs, then, of emphysema of the lungs, are a short inspiration with a feeble inspiratory murmur, and a loud, protracted, and wheezing expiration. (With crepitation, as the direct result of pure emphysema, (the râle crepitant sec à grosses bulles," of Laennec, and 
other auscultators who have followed him,) I have no practical acquaintance; and though I am not prepared, and am far from being disposed, to say, that it never does nor can exist, I believe I may truly state, that I never saw an unexceptionable case, in which it could be proved to have been present; and from multiplied observations I have no hesitation in expressing my assured conviction that the crepitant and other rattles, so constantly heard in emphysema, arise very generally, if not universally, from fluid secretions in the dilated cells, and smaller bronchial tubes.)

The loud and prolonged expiratory wheezing of emphysema is very remarkable, and may be satisfactorily explained as above. But not only is the expiratory wheezing extended vastly beyond the ordinary duration of the time employed in expelling the air from the chest, and greatly exceeds that occupied by inspiration; but in extreme cases it may sometimes be observed that this wheezing obviously continues after the act of inspiration has commenced. However apparently difficult may be the explanation, the fact is quite certain. It may be perhaps presumed to arise as follows:-

The great necessity and consequent effort of persons affected with extensive emphysema, is to gain a sufficient supply of fresh air. To accomplish this, they must exclude from the lungs as much as possible of the air which distends them. The act 
of expiration, as has been already explained, is chiefly effected by the elasticity of the ribs, which have already become considerably dilated, by the long-continued pressure exerted upon them by the enlarged lung from within. The elevation of the ribs upon inspiration, however small may be its extent, is quickly effected, and when their elasticity comes into play by the cessation of the inspiratory effort, their tendency to return to the situation from which they were raised is immediate. So far, then, as the ribs are concerned, the duration of the expiration in emphysema may be as short, or even shorter, than that of inspiration. Not so as regards the included air, which cannot escape from the multitudinous cells in which it is contained through its only outlets (the absolutely or comparatively contracted bronchial tubes) with a rapidity equal to that with which the ribs descend. The air, therefore, in the chest is temporarily compressed, and its tendency to escape does not cease when the ribs have ceased to fall. More than this, the elastic fluid within the chest does not regain its equilibrium, as regards density with the external air, until the elevation of the ribs actually occurs. This elevation does not, as in the state of health, take place immediately upon the contraction of the muscles of inspiration; nor, in consequence of the already largely dilated state of the chest, and the consequently unfavourable position 
in which those muscles are placed for efficient action, is it effected till the muscles have atready contracted to some extent.

Such appears to be the most probable explanation, as well of the sounds heard in emphysema, as of the curious fact to which reference has been made. The theory of the matter may be defective, or physiologically incorrect. The facts remain the same.

Supposing then that, together with general emphysema, there exists thickening, or obstruction of the larger bronchial tubes in one part, and dilatation in another, - contraction of the smaller canals here, and enlargement of them there,-the loud sonorous rattle arising from the contraction may be combined with the puffing or blowing of dilatation, the hissing of capillary narrowness, and the wheezing of emphysema. Under such circumstances, which are far from uncommon, the sounds, even independently of moist crepitating or mucous rattles, are of course exceedingly various. The note of a bass viol, the cooing of pigeons, the squeaking of young mice, the hissing of the escape of steam, snuffling and snoring, whistles and wheezes, puffing and piping, in various degrees and combinations, may be occasionally heard, and may be employed to characterize the strange and multifarious noises which are then audible.

(c.) Phthisis.-In the earlier stages of Phthisis, 14 
when but few tubercles are deposited, and no surrounding consolidation exists in the lung, a slight alteration of the respiration is often the only recognisable physical indication of the presence of the abnormal deposit. The tubercular bodies, whatever their origin, whether from inflammation, or from depraved nutrition, and whatever their exact seat, whether in the air-cells themselves, or in the vascular tissue which constitutes their boundaries, necessarily encroach upon the pulmonary vesicles. They consequently impede the free expansibility of the lung, and must, in some degree at least, detract from the elasticity of the organ. They must also exert some amount of pressure upon the capillary branches of the bronchial tubes. The consequence of this condition is, not merely that the soft breeze-like inspiratory murmur is perceptibly, however slightly, diminished, but that it is replaced by comparatively a harsh and hoarse sound. This latter sound, it is presumed, is dependent upon the pressure exerted upon the small and flexible bronchial tubes by the abnormal tubercular deposit, as the defective inspiratory murmur is upon the imperfect expansion of the pulmonary vesicles. From the same cause the intensity of the expiratory murmur is increased, and its duration prolonged. As was stated on a former occasion, whatever may be thought of the explanation here offered, or whether it be really correct 
or incorrect, it is certain that among the earliest physical indications of the presence of tubercles in the apex of the lung, are deficient inspiratory murmur, or harsh respiration, and more especially a prolongation of the expiration.

When, in a later state of phthisis, the portion of the lung affected becomes in some measure consolidated, partly from the increase of tubercles, but principally in consequence of surrounding pneumonia, or when pure pneumonia attacks a lung, and proceeds without ulceration to firm dry consolidation, or, as it is ordinarily called, tubercular infiltration, the air cells are entirely obstructed, and the vesicular murmur in the part is consequently absent. But in both these cases, in the tubercular combined with the pneumonic, and in the purely pneumonic consolidation, auscultation affords other signs than the mere absence of the respiratory murmur. Among the most important of these is bronchial respiration, or tubular breathing. The spongy tissue of the lung being converted into a firm solid mass, is changed from a very bad to a good conductor of sound, and the sound produced by the passage of air through the larger bronchial tubes is thus better conveyed to the surface of the chest; it also becomes more distinct from the absence of the natural sound of the respiration, which ordinarily interferes with, or conceals it. To state that bronchial respiration in such a case, or indeed 
in almost any case, arises from consonance, appears to me to be a gratuitous, and at the same time a very improbable assumption.

Does, then, tubular breathing necessarily indicate tubercular, or pneumonic consolidation? It will be seen in a future page that it certainly does not. It merely indicates this: that the bronchial tubes are either actually nearer the surface, or, acoustically speaking, are made to approach it by the intervention of an improved conductor; and, if the respiratory murmur be at the same time absent, that the cells of the lungs are either compressed or obstructed. To arrive at the cause of this physical condition, other means must be adopted than the mere auscultation of the breathing.

The character of tubular respiration varies according to the extent and situation of the part affected; that is to say, according to the size and amount of the tubes passing through, or near to the consolidated tissue. It is also, of course, modified in its purity and intensity, by the presence or absence of the respiratory murmur, and of morbid rattles, arising from mucus, blood, or other fluid in the tubes. Thus it may be of a shrill tone if the tubes are small; or it may be deep if they are large; but in either case it resembles the sound produced by blowing through a reed, a quill, or a straw, with little, or without any, compression of the lips, according to the perfection with which 
it exists. The more complete the consolidation, or the more pure the sound, the less obstruction does there appear to be to the transmission of air through the reed; the less compression of the lips does it require accurately to represent it; the more perfect, in fact, is the tubular character of the sound.

If, as it sometimes, though rarely, happens, a bronchial tube be dilated into a large cavity, and be in contact with, or very near to, the parietes; if, as it more frequently occurs, a moderately dilated tube be surrounded by a mass of consolidated lung, or be very close to the surface of the chest; or if, whether from tubercular degeneration or pneumonic softening, a large cavern be formed in the substance of the lung itself, and be not deeply seated, or have only diseased pulmonary tissue intervening between it and the parietes, then either a deeply-toned or blowing sound, or a sonorous and hollow kind of fizzing or hissing noise, takes the place of bronchial respiration. The former is named amphoric breathing, from its frequently exact similarity to the sound caused by blowing into a widemouthed bottle. The latter is termed fistulous breathing. It may be so denominated either from its resemblance to the sound caused by air permeating a reed which is split, or from its presenting to the ear of the examiner the impression of elastic fluid passing through a fistulous opening. Both 
of these sounds are also occasionally heard when a communication exists between a bronchial tube and the pleura.

Nearly allied to these sounds is the metallic respiration, or metallic resonance of the respiration. It sometimes accompanies one or other of them, and very generally exists in very nearly similar physical conditions of the lung, bronchial tubes, or pleura. It gives an impression to the ear of a large cavity, equally with the amphoric breathing; butit possesses a ringing or brazen twang, which may be perhaps fairly represented by blowing through a tube into a moderately-sized bell or glass vase, the sides of which vibrate slightly, or, as it is called, ring, from the blast; or, more correctly still, by holding a large wide-mouthed glass vase close to the ear, when, instead of the murmuring of the sea-shell, a metallic ringing will be heard.

It is often, but not necessarily, associated with another sound, (metallic tinkling,) and appears to depend upon the free vibrations of air against, and their reverberations from, the resonant parietes of a large cavity. The whole of these three sounds are generally, if, indeed, they be not uniformly, confined to the act of inspiration.

In what, then, consists the difference of the cause of these sounds? What are the physical conditions necessary for the production of each? They are similar, but not identical, in character. They give 
to the ear an impression of air passing into a cavity of considerable size; they likewise exist under circumstances nearly similar, and they sometimes replace each other. Though, therefore, the physical conditions necessary for the production of each must be very similar in all of them, it is clear they are not identical. In what, then, does the difference consist? To this I, at least, am not able to reply with confidence. I may, however, state that I believe the difference of the fistulous, and the amphoric respiration, to depend upon the size and form of the opening leading into the cavity of the lung or pleura; that the opening is small, or obstructed when the former, and comparatively large when the latter sound is heard; and therefore that amphoric respiration may become fistulous, by the tube, through which the air gains admission into the free space, becoming obstructed with secretion, or being diminished in its calibre from any other cause, and that fistulous may be converted into amphoric breathing by the removal of such obstruction, or by the tube from other causes becoming enlarged. I conceive also that the metallic ringing of the respiration is dependent rather upon the nature of the walls of the cavity itself, than upon the form or size of the opening leading to it, and that it is from the vibration of these walls being communicated to the air contained within them, that the peculiarity of the tone arises. Hence I believe it happens that metallic respiration is very rarely 
heard unless some portion at least of the walls of the cavity are formed by the solid parietes of the chest, or by a thin layer of firmly adherent pleura lining them. The determination of this question, however, is fortunately not one of importance in a practical point of view, whatever interest it may possess theoretically.

The auscultatory signs produced by Malignant disease of the lung are exceedingly variable, according to the situation, amount, and distribution of the morbid deposit. Sometimes malignant tubercles are irregularly scattered through the lung; but as they give rise to no general symptoms, the chest is frequently not particularly examined, or, if it be examined, it may, from the tubercles being surrounded by healthy crepitant lung, afford little or no indication of their presence. At other times, disease of much less extent may, from its position near to, its pressure upon, and its obstruction of, a considerable branch of a bronchial tube, produce a loud, hoarse, and harsh tubular breathing, or wheezing; and from its merely mechanical effect, may at the same time give rise to great general distress.

In other forms of the complaint, when the malignant deposit exists in a large isolated portion of the organ, and the bronchial tubes are not materially compressed or pushed aside, the physical signs, as might indeed be expected, are much the same as those arising from consolidation of the lung from 
other causes, and, as regards the breathing, are almost confined to the absence of the respiratory murmur and the presence of bronchial respiration. When, again, the malignant mass is so large, or is so situated, as to push aside the lung altogether, or to compress and actually to close the bronchial tubes, all sound of respiration, whether vesicular or tubular, ceases in the part affected; excepting so far as it is conducted by the consolidated mass from the lung of the opposite side of the chest, or a comparatively healthy portion of the lung on the same side. The sounds, under such circumstances, appear not to be close to the ear, but to come, as in truth they do come, from a distance.

Such, then, are the chief and the most important of the $d r y$ sounds connected with the respiration in disease. For the sake of distinctness, they have been mentioned separately. Separately they often exist; but the student must recollect that they are more frequently variously combined with each other, and with the different moist sounds or rattles, which will be spoken of hereafter, and which often detract from the clearness of their character, and consequently render them more difficult of discrimination than when they exist alone.

3. Dry sounds in diseases of the Pleura.

The smooth, moistened surfaces of the pleura, during the respiratory movements of health, soft- 
ly glide over each other without giving rise to any sound. They act like a beautiful piece of machinery in good trim and well oiled.-That they do so glide over each other in health, appears sufficiently evident from the effects of disease, when the machinery is out of order; the surfaces lose their polish, or, it may be, are not supplied with the amount of oil necessary to make them play easily upon each other.

The first physical effect of inflammation in a serous membrane is supposed to be to render it abnormally dry. It is presumed that this inflammatory dryness may itself, independently of any effusion, give rise to a morbid sound, just as the absence of oil on a piston, or in a joint, may be the cause of an unusual one in machinery. This is possible; but it may be questioned whether the mere dryness of a serous membrane coexisting with, and the pure result of, the first stage of inflammation, has been ever proved to have produced such an effect.

When, however, effusion has taken place, and the serous membranes are coated with an albuminous layer, however thin; when the smooth, polished surface of the pleura is replaced by a rough and plastic one, it is not merely probable, but in numerous cases it has been proved, that the surfaces of the membrane, in place of gliding smoothly and noiselessly over each other, rub roughly against 
each other on the way, and on that account by their motion produce a sound. Two rough surfaces, in fact, are now in contact, and the friction of one against the other, when they are moved, as in the act of respiration, like that of other rough surfaces, makes a noise, or in other words, produces a vibration of the substances of which they are composed. This sound is appropriately called pleuritic rubbing, friction sound of the pleura, or "frottement ascendant et descendant." This last term has been employed in consequence of the sound usually, but far from universally, accompanying both the elevation and the falling of the ribs.

The sound varies much in character. This variation probably depends as well upon the quality as the quantity of the solid matter intervening between the two surfaces of the pleura. When the effused lymph is recent and soft, it appears as if the opposed surfaces stuck together by a slightly adhesive material. The sound which results from the motion of the parts, under such circumstances, is interrupted and jerking. It closely resembles that which may be produced by pressing firmly together, and moving slowly over each other, the palms of the hands, moistened in the slightest degree possible with perspiration. It may be also likened to the stiff or crisp noise caused by squeezing a packet of arrow-root, or by treading upon snow which is wet or melting. 
When the plastic effusion, in consequence of the separation, or the absorption of some of its liquid constituents, has become less moist and adhesive; when a solid, dry, rough coating is left upon corresponding portions of the pulmonic and costal pleura, a simple rubbing noise is heard upon inspiration and expiration, which may assimilate to that arising from the attrition of two pieces of cloth. At other times the sound is very loud, harsh, and grating ("ractement,") and can often be heard and felt by the patient himself, as well as by the physician and the attendants, if the hand or ear be placed upon the chest. This harsh, rough, grating sound ordinarily, but not universally, coexists with the inspiration alone; it may be compared to that arising from the attrition of two pieces of rough wood. In some instances, on the contrary, the pleuritic rubbing is heard only at the end of the expiration.

There is yet another peculiarity in reference to the sound arising from morbid deposit in the pleura. It sometimes assumes a curiously creaking, or squeaking character, and really most exactly simulates the noise caused by bending a piece of stiff leather. ("craquement de cuir.") In this case it is ordinarily heard only towards the end of the inspiration; a circumstance that certainly renders probable the opinion, that it depends upon the stretching and tension of old adhesions. Sometimes, in- 
deed, the different varieties of pleuritic rubbing, excepting perhaps the first, are heard only at the end of a forced inspiration, when the lung is most distended with air, and when, if the pressure be not greater, the extent of contact between the two folds of the pleura is increased.

The first and second varieties of pleuritic rubbing above referred to, may certainly exist either previously to the effusion of fluid, or subsequently to its absorption; but it is at least probable, that the harsher kinds of sound are connected with chronic disease, and therefore ordinarily follow the absorption of fluid. Immediately, however, that fluid intervenes between the two surfaces in the case of recent disease, or so long as any remains behind in that of longer date; while, in fact, the presence of fluid prevents the contact, and consequently the friction of the two opposing surfaces of the pleura, no rubbing sound can be heard.

In the earlier stages of phthisis, the murmur of inspiration below the clavicles and in the acromial region often presents an irregularly interrupted, jerking, or catching sort of sound ("saccadé.") It rather accompanies, than supplies the place of, the respiratory murmur. Its origin is not certain, but it appears most probable that it is connected with a roughened state of the pleura, the result of effusion, and that it is, in fact, a modification of pleuritic rubbing. 
At other times, though much more rarely, there is heard during the inspiration, in the same situations, a soft rustling, or crumpling noise, approaching to that produced by squeezing together soft gauze paper-pulmonary rustling ("froissement pulmonaire.") It has been so named, on account of its supposed connexion with an alteration of the pulmonary tissue; but, like the former sound, it appears to myself to be more probably connected with some change in the serous membrane, which prevents the two layers gliding over each other with perfect smoothness, and without noise. Neither of these sounds are of much value in a diagnostic point of view, especially as their origin is uncertain. But as they indicate some change in that part of the lung, or of its covering, in which tubercles are, in a vast majority of instances, first deposited, they should not, in dounbtful cases, and in connexion with other physical signs, be altogether neglected. I believe that they result from changes in the pleura, and that these changes may depend upon the presence of tubercles, either in the lungs, or in the membrane itself. It is, however, right to say, that I have no solid grounds for this opinion, as I have never had an opportunity of investigating their origin at the dissecting table; nor, indeed is it likely that such should be the case, as they are, comparatively, so rare, and of so little importance (the second more especially,) 
and occur only in so early a period of phthisis, that the opportunity of investigating the physical condition upon which they depend must be exceedingly rare indeed.

Where either fluid or solid matter exists in the pleura, to an extent sufficient materially to compress the spongy tissue of the adjoining lung, the respiratory murmur, of course ceases in that part, in consequence of the air-cells beneath the effusion not being expanded during inspiration. The respiratory murmur being absent, and the spongy tissue being compressed, and, by its compression, being converted into a body of superior conducting power, the sound of the air passing through the bronchial tubes is conveyed to the surface. Thus tubular breathing, or bronchial respiration, together with the absence or the distance of the respiratory murmur, becomes a valuable sign of effusion into the pleura.

It is, however, a sign of only a limited amount of fluid, excepting under peculiar circumstances, and in particular parts. For, if the lung be not adherent to the parietes of the chest, and if it be otherwise in a healthy condition, as the fluid accumulates from below, it presses the lung more and more upwards. It is consequently farther and farther removed from the general surface of the chest; so that at length, when the fluid is in large quantity, it is compressed into a very small com- 
pass close to the spine. Under such circumstances it is clear that the bronchial tubes themselves; by which the sound is primarily and best conducted, must, together with the lung, be far removed from the greater portion of the parietes. The prime conductor of the sound, therefore, being far removed, though the fluid be a better conductor of sound than the natural spongy structure of the lung, not only is the respiratory murmur lost, but the bronchial respiration itself, excepting at the root of the lung between the shoulder-blades, and, it may be, below the clavicles, is greatly diminished, and in many cases becomes quite inaudible.

Some exceptions, as has been already hinted, exist to the uniform correctness of these general statements. The first of these depends upon the gravitation of the fluid. When the fluid is not in large quantity, is not confined by adhesions, and is not combined with a large proportion of plastic or albuminous matter, it may change its situation, according to the varying position of the patient's body. The part, therefore, in which no respiratory murmur is audible, and in which tubular breathing is distinct, while the patient is supine, may be destitute of tubular breathing, and afford a very tolerable respiratory murmur, when he turns upon his face. This fact is much more commonly observed in hydrothorax, than when the effusion has resulted from pleurisy, in which it is certainly not so common as it has been represented to be. 
The next exception arises from adhesions. Though the fluid may be in large quantity, and though the lung may, in the main, be greatly compressed, and, as regards its chief expanse, be far removed from the general parietes, it may be bound down at one or more parts to the costal, or phrenic pleura, by old adhesions, too firm and strong to yield under the pressure of the fluid. At this part, then, the lung is not removed from the surface, and though no trace of pure inspiratory murmur may be distinguishable, it is yet quite possible, and indeed it often occurs, that very well-marked bronchial respiration is heard under such circumstances in such parts, though they be far removed from the root of the lungs.

It may be here observed, though the observation can be scarcely regarded as an exception to the general statements previously made, that, in all these cases, bronchial respiration can be heard, even more distinctly than naturally, in the interscapular region of the affected side. It is more loud and pure upon the diseased than upon the healthy side, in consequence of the fluid pressing the large bronchial tubes against the solid parietes of the chest, and excluding the air from the surrounding and intervening lung.

The last exception that need be mentioned is the mostt curious one, and by far the most difficnlt of explanation. In some cases, which are not so 
rare as it has been by some authors supposed, it happens that though the pleura is actually full of fluid, and may be proved to be so by inspection after death, not only is tubular breathing general, but a shrill resonance of the voice is distinctly audible over the whole side. Though the lung be compressed into the smallest space, and the bronchial tubes be therefore far removed from the solid parietes, in all parts excepting in the neighbourhood of the spinal column, still their vibrations are communicated to those parietes, and from them to the ear. How does this happen? It is, indeed, difficult positively to assert how; and because difficult to explain, the fact itself may be questioned. The same thing, however, in regard to the voice, as well as to the tubular breathing, has occurred in persons from whose chests, immediately after the examination and observation of these signs, several pints of fluid have been drawn off by paracentesis. In reference to such cases, then, there can be no doubt.

How, then, are these circumstances to be explained? It is acknowledgedly one of those cases in which ordinary acoustic principles appear, (most probably from our not being aware of the whole or the exact circumstances of the case,) to be insufficient for the satisfactory explanation of what is occasionally heard in the practice of physical diagnosis. 
If a conjecture might be ventured upon the subject, it would be this:-So long as there exists any portion of the lung which is capable of farther compression; so long, therefore, as the parietes are not tense, and the fluid contained within them, though in large quantity, is moveable; so long is bronchial respiration inaudible over the general surface of the side. But when the compression is great enough to render the parietes and the fluid contained within them tense, but not so great as to flatten or absolutely to close the larger bronchial tubes, then the vibration of those tubes is communicated to the spine, or to the bony parietes, against which they rest, and thence, as in a well-braced drum, to the walls of the entire cavity. The fluid is so tightly pressed, that it moves as a solid, and conveys the vibrations of the sound as a solid to, and with, the solid walls. As to the breathing being sometimes not heard, at the same time that the voice is distinctly audible, and even shrill, this probably depends upon the greater or less extent of the vibrations from which the two proceed.

In the former edition of this work I spoke doubtfully and hesitatingly in reference to this curious subject, and as if it were of very rare occurrence, as it has indeed been represented by most authors; seeing that my attention had been directed to it in only a few instances. From many cases, however, which I have since examined pre- 
viously to the operation of paracentesis thoracis, and from many others, in which almost equal evidence existed of the presence and accumulation of fluid in the pleura, I am quite certain that it is not uncommon; I feel assured that it exists as the rule; and I very much question whether, if some accidental circumstance does not exist to prevent its development, it is not generally present when one side of the chest is fully distended with fluid.

When a communication exists between one or more bronchial tubes, and the cavity of the pleura; whether the communication be the result of a tubercular abscess bursting into the serous membrane; of a collection of purulent fluid in the pleura emptying itself through the lung: or of gangrene, which are the three most common causes of pneumothorax; a considerable quantity of air usually finds its way into, and continues within, the serous sac. Here then, if at any time, might be probably presumed to exist, the fistulous breathing, the amphoric respiration or bottle blowing, and the metallic resonance, which have been previously mentioned, while speaking of the diseases of the lung; and here it is that they do, in fact, exist most frequently and in their greatest perfection. - It is unnecessary to attempt to describe them again; they must be perfectly known and thoroughly appreciated. To hear them once will conduce more to their future recognition than whole pages of descriptions and 
similitudes. It cannot, either, be necessary again to explain their origin. It is sufficient to say, that they indicate with tolerable certainty a large cavity with resonating walls, and a free ingress of air. A caution is here requisite, to guard the student against a very important, and in some respects not a very unfrequent source of fallacy. The whole of the sounds last described, and particularly that mentioned in the next paragraph, may be distinctly heard in examination of the chest, and appear to be close under the ear; while they in reality exist in the abdomen, and arise from the motion of air and fluid in the distended stomach. The sounds may, in truth, be under the ear, in consequence of the largely distended stomach, by pushing up the diaphragm and displacing the lung, coming into near contact with the ribs, as high even as the fourth or fifth; or, they may be so in appearance only, in consequence of their being conveyed through the lung, increased in its conducting power by consolidation, or by the compression resulting from fluid effusion.

There is another sound connected with diseuse of the pleura, as well as of the lung, the tone of which is assuredly often dry, and which may possibly, and, as I believe, actually does, sometimes arise independently of the presence of fluid. But as "metallic tinliling," the sound to which I refer, is certainly most frequently connected with the vi- 
bration of fluid reverberating in air, and as it is not perhaps proved that it ever exists independently of the presence of fluid, it may be better here barely to refer to it, and reserve its fuller consideration for the next section.

4. Moist sounds in diseases of the Air-passages, and Lungs.

With the view of relieving the student of some portion of the perplexity, with which he may possibly have hitherto contemplated the names of the various rattles, or moist sounds which occur in disease, a few simple statements will be made in reference to the whole of them, as a class, previously to either mentioning them individually, or particularizing the complaints during the progress of which they arise.

All the moist sounds heard in diseases of the respiratory organs, it may be then first remarked, arise from the passage of air through fluid, or the admixture of air with fluid. They may occur during the act of inspiration, or of expiration; may be situated in the larger, or in the smaller bronchial tubes, which may contain mucus, blood, serum, or pus; they may originate in a portion of disorganized lung, or in a cavity, either pulmonary or pleural; they may be produced independently of any respiratory act by the simple motion of body; they may vary in tone and in character, in site and 
in pathological origin; but their cause, physically speaking, is the same in all. It is the mechanical admixture of fluid and air. Separately, either of these is incapable of giving rise to moist sounds. Air will certainly not do so, and fluid cannot without the presence of air. In a tube replete with air, no moist sound will be produced by agitation, neither will a cask quite full of fluid, however shaken, give rise to any rattling or squashing noise.

The moist sounds similar, and for the most part identical, in their mode of production, have been differently named, according to the size of the bubbles producing them, or, what in a great majority of cases amounts to the same thing, according to the calibre of the tube through which, or the sizes of the cavity into which, the air passes. Thus the bubbles of air arising from its admixture with fluid in the trachea are either actually of greater dimensions than in the bronchial tubes, and in the larger than in the smaller tubes; or the different extent of the space in which the bubbles are formed and burst, produces a corresponding difference in the resulting resonance, which causes them to appear to be so.

It would, I think, be desirable, as well as more correct, to confine the term rhoncus to the dry sounds, and the terms râle or rattle to the moist sounds. I have, however, in this as in some other instances, preferred to use the terms, as they gene- 
rally are used, indifferently, than to perplex the student by the introduction of fresh distinctions.

When the bubbles are very large, or their resonance is very full, as they occur in the trachea, or in a tubercular cavity, they are called tracheal rattles or gurgling. When they are somewhat smaller, as in the bronchial tubes of first or second size, they are called mucous or bronchial rattles. When they are still smaller, as in the finer divisions of the bronchial tubes, they are termed mucocrepitating or sub-crepitant rattles; and when in the air-cells themselves, or in the capillary branches in immediate connexion with them, they are named crepitating rattles.

Let the student imagine the bubbles, the bursting of which gives rise to tracheal rattles or gurgling, to be the size of a horse bean; those of the mucous rattle, the size of a pea, or a swan shot; those of the muco-crepitating rattle, the size of a tare, and of a millet-seed; and those of the crepitating rattle, the size of a poppy-seed; and he will form no very incorrect notion of the relation of these sounds to each other; and, bearing in mind the manner in which they are produced, he will, in most cases, not be very far wrong in his assumption as to their seat and their import.

The moist sounds vary as to their distinctness, tone, and quality, according to the facility or difficulty with which, and the medium by which, the vibrations producing them reach the surface of the 
chest; or, in other words, according to the goodness, or badness, or character of the conductor intervening between their source and the parietes. They are, for example's sake, more shrill and loud when the lung is consolidated, than when the pulmonary tissue is in a healthy condition. They also vary, in a slight degree, according to the nature of the fluid in which they originate. They are somewhat more shrill, or sharp, if produced by blood in the tubes, than by serum, and by stiff tenacious mucus, than by ordinary bronchial secretion.

The preceding statements are, it is hoped, sufficiently simple, and must, it is believed, be intelligible to all; yet it is both hoped and believed that, by bearing them constantly in mind, the student will be enabled, not merely easily to follow, and to understand the succeeding remarks, which can be regarded as simply an extension of them, and an application of them to particular cases; but that he will be himself competent to recognise, and in some degree to appreciate the value of many of the fluid sounds, which he hears in his examinations of pulmonary disease.

When, either in addition to, or independently of ulceration, constriction, or compression, fluid is present in the larynx or trachea, the passage of the air through the fluid, in the acts of inspiration and expiration, and the consequent formation and burst16 
ing of bubbles, produces a loud and loose bubbling, or gurgling noise. It may be, and generally is, audible without approximating the ear, or applying the cylinder to the throat; but it is most distinctly heard, and its situation is most accurately defined, by means of the stethoscope, gently and evenly placed upon the side of the neck. The sound is a little, but only a little, modified by the quality of the fluid in which it originates. It is rather sharper in tone when the fluid is thick and viscid, than when it is thin and bland. Thick purulent, or muco-purulent matter, and tough viscid mucus, give rise, therefore, to a sound rather more shrill than common bronchial mucus, watery phlegm, or blood.

The difference arising from the quality of the fluid may be fairly illustrated by blowing through a straw into simple water, and into a somewhat denser fluid, as soap and water, thin treacle, or syrup. The moist tracheal sound is the same as that which often, in the last few hours of life, results from the failure of the nervous energy, is familiarly called "the rattles," and by uninformed persons is very generally, but incorrectly, considered to be the certain precursor of death. By auscultators it is called the "tracheal rattle."

When the bronchial tubes of first or second-rate size contain fluid, whether that fluid be blood or mucus, the bubbles which arise from the transmis- 
sion of air through them, appear to be smaller than those existing in the trachea and larynx. The resulting sound is called mucous rattle ("râle muqueux.") It is present whenever fluid exists in the tubes, and the free passage of air admits of the production of bubbles. The sound is most commonly produced by the presence of bronchial mucus in the tubes. It is hence called mucous rattle. It may, however, arise from other fluids. Its designation is not changed on that account, but whether originating in mucus or pus, in blood or serum, it is still called mucous rattle, as the name is generic, and has reference rather to the character of the sound, and to the part, than to the nature of the fluid in which it originates.

It must not, however, be supposed that in the mucous rattle, the bubbles are, or appear to be, all exactly of the same, and that a medium, size; as independently of the difference of the calibre of the tubes themselves, it is clear that small bubbles may arise in a large tube, though large bubbles cannot exist in a small one. The name and the description are derived from the general and most prominent character of the sound, which gives an impression to the ear of the bursting of bubbles of a moderate size.

When general dilatation of the tubes is present, the bubbles appear to be larger than usual; especially when existing in those parts of the lung in which the tubes are naturally small. 
When, instead of general dilatation of the tubes to a moderate extent, there exists such an extension of the walls of one, or of several of them, as to form considerable, though it may be irregular, cavities, and when cavities are present in the lungs, whether those cavities result from tubercular disease, from gangrene of the lung, or from pneumonia, a large tracheal rattle, or more properly, gurgling ("gourgouillement") is heard in the part affected upon each act of inspiration. Are, then, it may be asked, tracheal rattles and gurgling precisely the same thing? Not exactly. They are very similar, but not identical. Independently of tracheal rattles being, strictly speaking, confined to the larynx and trachea, though both the tracheal and gurgling rattles appear to result from the bursting of large bubbles of air, and both, therefore, also appear to proceed from a space of considerable size, there is still a slight difference between them: Gurgling gives an impression to the ear of a thicker or more dense fluid than the ordinary tracheal rattle. The sound, too, generally corresponds with the actual condition of the fluid in which it originates, or of the medium by which it is conducted, and sometimes modified. For, as the rattles in the trachea usually proceed from common bronchial mucus, or from blood, gurgling is ordinarily produced by pus, thick muco-purulent or softened tubercular matter. Does true gurgling, then, neces- 
sarily indicate the presence of either a largely dilated tube, or a cavity in the lung? It certainly does not. It generally does so, but not necessarily. For if a bronchial tube of moderate size, or a tube dilated in only a slight degree, contain thick purulent mucus, and be surrounded by a dense mass of consolidated lung, the sounds which result from the passage of air through the thick fluid in the tube, and which, under ordinary circumstances, would give rise only to a mucous rattle, will be so increased in loudness and fulness, by the improved conducting power of the solid mass, as exactly to resemble the gurgling of a cavity. Let the student, therefore, be careful not to pronounce too confidently upon the existence of a cavity, from the presence of gurgling alone, or he will assuredly be liable to error, and will sometimes find himself mistaken. Gurgling, then, is a large and resonant mucous rattle not existing in the trachea, but nearly resembling the tracheal rattles. It commonly arises from a cavity in the lung, or from a largely dilated bronchial tube; but it may proceed from a tube of normal size, which is filled with thick fluid, and is also surrounded by a mass of consolidated lung.

When the smaller tubes are the parts solely or principally diseased, or rather when morbid secretion, or other fluid, is present in the minute branches of the bronchial tree, the moist sound, which is produced by the passage of air, appears to arise 
from the bursting of bubbles smaller than those constituting the mucous rattle, and larger than those which are next to be mentioned, and which give rise to crepitation. The sound is therefore appropriately called the muco-crepitating or sub-crepitant rattle. Excepting as regards the apparent size of the bubbles producing it, and the calibre of tubes in which it exists, it resembles the mucous rattle, to which it bears much the same relation as the sibilating does to the sonorous dry rattle or rhonchus. Like the mucous rattle, it is rendered more shrill and sharp by the presence of consolidation of the lung, and from causes similar to those which have been already mentioned, when speaking of the mucous rattle and gurgling.

When fluid is present in the air-cells of the lungs themselves, or in the capillary bronchial tubes, the sound which results from the admixture of air with the fluid appears to arise from the bursting of bubbles still smaller than those previously referred to; and if the fluid be strictly confined to the cells, the bubbles, like the cells and capillary tubes themselves, appear all to be nearly equal in size. It matters not what may be the nature of the fluid, - supposing it to be situated in, and confined to, the pulmonary vesicles, and capillary tubes,-whether it be blood, serum, or viscid mucus, the sound which is heard upon inspiration is like that produced by the bursting of bubbles of very small and of equal size. 
Whether, therefore, the cells are partially filled with serum, as in odema of the lung,-with blood, as in apoplexy of the lung,-or with thick viscid mucus, as in inflammation of the lung, a very fine sound, similar to that which is heard when salt is thrown upon the fire, and is called crepitation, arises from the intermingling of the inspired air with the fluid so situated. It is hence called the crepitating or crepitant rattle ("râle crepitant.") When existing in the greatest purity, it is most correctly represented by rubbing a lock of hair between-the finger and thumb close to the ear.

Is there, then, any difference between the crepitating rattle of odema, pulmonary apoplexy, and pneumonia? It would be, perhaps, incorrect, or at least questionable, to state that a difference does generally exist between them; but in practice it is found that a difference may in some cases, at any rate, be appreciated. The truth is, that the pure crepitating rattle is very rarely heard, either in pulmonary apoplexy or in oedema. It is generally combined with the muco-crepitating rattle of the smaller tubes, into which, as well as into the aircells and capillary tubes, the serum or blood is almost always likewise effused.

As the air intermingles with the fluid in the tubes upon its exit from, as well as upon its entrance to, the lungs, the mucous and muco-crepitating rattles are heard during the expiration as 
well as during the inspiration. The same occurs, and for the same reasons, when serum or blood is present in the smaller branches of the tubes. But, although it be quite true that capillary bronchitis very frequently exists in combination with pneumonia, and though, when such is the case, it may be difficult, or even impossible, to state with certainty, and from the sound alone, that the crepitating and muco-crepitating rattle may not arise from pulmonary odema or apoplexy; yet it is also true that pneumonia is often present without any affection, or without any physical indication of any affection, of the small tubes.

It is in such, and in such cases only, that the crepitating rattle, as the result of disease, is heard in its purity and perfection. It is then very small, very crisp, very sharp, very equal, and heard only at the end of the inspiration. The only sound which, to the best of my knowledge and belief, exactly resembles it is one which is sometimes heard for a short time, i.e. during a few inspirations, under circumstances which render it probable that no disease at all exists, either in the lung or the pleura. When, for example, a person who has been for some time lying on his back, and breathing quietly, is submitted to examination, and directed to take a deep inspiration, it not very unfrequently happens that at the very end of the inspiratory effort, there is heard, at the posterior 
part or base of the lung, a very pure and perfect specimen of crepitation or crepitating rattle. The examiner feels assured, if fever be present, that he has a case of latent pneumonia. If he has used his unguarded ear, to avoid fallacy he puts his hair aside; if the stethoscope have been employed, he may apply the naked ear to the chest, to make "surety doubly sure;" or he may direct a pupil's attention to the sound, as a good example of what he may have been long searching for. But the pupil does not hear it. The examiner himself tries again, but he can hear it no more. It is gone, and pure respiratory murmur has supplied its place. I have not unfrequently been thus deceived myself; and my pupils have also been disappointed of an expected novelty; the fact being, that the valued, but really rare sound, in this case prevails only during two or three, at the most half a dozen, inspirations.

What is the source of this sound? Present usually at the base of the lung, heard only at the end of a deep inspiration, and disappearing altogether after a few forced expansions of the chest and of the pulmonary cells, it appears at least probable that it may arise from the unfolding, or the separation, of the delicate walls of some pulmonary vesicles, which, from not being used in ordinary respiration, may have become somewhat dry, and may thence, like a dried bladder, give rise to the sound upon their insufflation. 
The crepitation of emphysema is larger and looser, and more unequal, than that of pneumonia; as before stated, it most probably arises from the presence of serum or of mucus in the dilated air-cells and the capillary tubes.

The attempt, however, to describe these minute differences of sound is almost useless. The sounds must be heard to be appreciated; no description, however exact, and however minute, can render them practically available to the uninitiated. Auscultation cannot be learned by book-except for the purpose of passing an examination. The student must go to the bed-side,-must go frequently, stay long, and work hard. Without this, he cannot become a practical auscultator-without this he cannot become a skilful practitioner-without this his opinion will be ever of little value in the diagnosis of diseases of the chest.

In pneumonia, then,-in oedema, in apoplexy, and in emphysema of the lung,-crepitating rattles may be heard. In all, the sound probably arises from causes nearly similar, viz., the effusion of fluid into the capillary tubes and the cells of the lung, and the intermingling of the inspired air with that fluid. But in all of them, excepting in pneumonia, the crepitating rattle is almost always combined with some amount of muco-crepitation, arising from the presence of fluid in the smaller branches of the bronchial tubes. In pneumonia, 
the sound may be likewise so combined, but it frequently occurs alone. 'It is the especial characteristic of inflammation of the pulmonary tissue; and if perfectly pure, equal, persistent, and existing only at the end of the inspiration, it may perhaps be still regarded as nearly pathognomonic of that complaint.

Crepitating rattle, then, in its purest form, is present in, and is especially characteristic of, the first stage of pneumonia, when the air-cells are still unclosed, but contain fluid secretion. When the cells are quite obstructed, either from the tumefaction of their vascular parietes, from the deposit of solid matter within or without them, or from both causes combined, all vesicular sound, all sound, that is to say, dependent upon the admission of air into the cells, necessarily ceases in the part affected; and tubular breathing, or bronchial respiration, supplies its place.

When the disease is on the wane, when the abnormal thickening is diminished, or when the deposit is removed, and the cells become again capable of admitting air, the physical condition of the part is very similar to that existing previously to consolidation; the physical signs are consequently similar. The crepitating rattle reappears, and, in the first instance, in a very pure form; but, as the complaint gradually declines, the rattle becomes more and more loose and large, and therefore more 
and more resembles the muco-crepitating rattles of bronchitis of the small tubes, which it also resembles in accompanying the act of expiration, as well as that of inspiration. This is the "râle crepitant redux" of the French auscultators.

When, instead of retrograding, the disease goes slowly on to induration and contraction of the pulmonary tissue, tubular breathing will probably become permanently established in the part; and if, in the progress of time, the bronchial tubes entering it become dilated, amphoric breathing, or, when fluid is present in the tubes, gurgling, will be constantly heard therein.

When, in place of the disease terminating in resolution, and when the lung, in consequence of the unhealthy, unorganizable nature of the deposit, instead of becoming indurated and contracted, begins to break up from the softening of the inflammatory product, air is again admitted to the anterior of the lung, and, by mixing with softened and semi-fluid matter, gives rise to a sound which is very peculiar, and which, as far as my experience extends, exists only in that condition of the lung, and in a similar condition occurring in the progress of phthisis. This sound is neither crepitating nor muco-crepitating rattle, nor gurgling, but it is a combination of the two latter sounds; or rather, it is the crepitating rattle altered in character by the thick viscid quality of the fluid into which the air 
passes, and by the increased conducting power of the surrounding consolidated mass. To the ear, indeed, it appears to be connected with precisely those physical conditions, and to arise from exactly that state of the respiratory organs, which actually exist; viz., the passage of air into a soft mass, such as the softened rotten lung, reduced nearly to the consistence of hasty pudding or thick treacle.

It is in fact a sharp, shrill, and loud muco-crepitating rattle, and may be not inaptly represented by the sound produced by passing the saliva to and fro through the teeth with the mouth closed. It is, indeed, in other words, a diminutive gurgling, or a large, moist, resonant crackling.

As the lung continues to break down, the bubbles become larger and larger, and the rattle, though still unusually shrill, assumes more and more of a mucous character; till at length, in consequence of the removal, by expectoration, of the semi-fluid mass constituting the disorganized lung, a cavity is formed, and amphoric breathing, and gurgling, supply the place of other sounds. It must, however, be recollected, that though a cavity be formed, and the physical signs of such cavity be sufficiently. evident, it may be still surrounded by pulmonary tissue in various stages of degeneration; and that this disorganized tissue may give rise to many of the fluid sounds, previously mentioned, which may 
more or less interfere with the distinctness of the gurgling and amphoric respiration.

In the early stages of Phthisis, the first moist sound that is heard is most commonly a fine and delicate muco-crepitating or subcrepitant rattle, audible only at the end of the inspiration, and at the commencement of the expiration; it appears to be situated in the smallest branches of the bronchial tubes, and is most probably dependent upon slight bronchitis, or increased bronchial secretion, the result of the irritation produced by the presence of tubercles.

This is commonly, as I have said, the earliest moist sound which accompanies the deposition of tubercles. But it sometimes happens, that even previously to the appearance of this sound, there is heard in those parts of the lung in which the tubercles are well known to be almost always first deposited in the ordinary form of phthisis, a sort of click, which, from the nature of the sound, may be presumed to arise from the obstruction afforded to the passage of the air by a portion of adhesive mucus, or other sticky substance, in a large tube. Even when this sound is present, which is not very frequently, it is only heard occasionally; that is, it is not heard at each inspiration, and perhaps not oftener than once in four or six inspirations. Its origin is doubtful, and, as it is somewhat unusual, and therefore of comparatively little diagnostic im- 
portance, it is unnecessary farther to dilate upon it, excepting for the purpose of stating that it may be represented by the sticky noise produced by the removal of the tongue from the roof of the mouth.

As in the progress of phthisis, the number of tubercles increases, or, more particularly, as they become surrounded by pneumonia, the bronchitis and its accompanying physical signs increase also. The muco-crepitating rattle becomes more general; that is, it occupies a greater portion of the inspiration, and it also becomes more shrill, as it is now better conducted by the greater or less consolidation of the surrounding tissues. It is also sometimes mixed with the genuine crepitation of pneumonia, and with the mucous rattles of bronchitis of the larger tubes.

From this stage of the disease, the moist sounds of phthisis so generally resemble those which occur in the unhealthy form of pneumonia, accompanied with scrofulous or unorganizable deposit, which sounds have been previously described, that it is scarcely necessary that they should be again mentioned. It may be, however, stated, that the shrill muco-crepitation, the loud mucous rattle, and the gurgling, succeed each other with varying celerity in different cases, and are variously combined accordingly as smaller communications with the bronchial tubes, or larger cavities, are produced in the destructive progress of the disease. 
It may also be not irrelevant again to remind the student, that when little, or scarcely any, indication of disease can be discovered below the clavicle of either side, a cavity of moderate size may be present in the very apex of one or both lungs, the physical evidences of which can be detected only by applying the stethoscope at the side of the neck above the clavicles, or more especially at the highest part of the acromial region posteriorly.

5. Moist Sounds in Diseases of the Pleura.

It has been already stated, that when fluid exists in the pleura, whether the quantity be large or small, unless air be likewise present, no moist sounds are produced, either by the act of inspiration, or by the motions of the body. It may, however, perhaps, be questioned whether the sticky noise which results from the friction of recently effused plastic matter, or the somewhat similar sound which arises after the fluid portion of a pleuritic effusion has been in a great measure absorbed, may not be fairly considered to constitute an exception to this general statement; as the impression given to the ear is certainly not that of the friction of perfectly dry substances. It appears at least doubtful if any thing more nearly approaching to a moist sound than this ever arises from simple pleurisy, and it is exceedingly questionable 
if sounds simulating the mucous, and even the crepitating rattles, have been ever demonstratively shown to originate from fluid, or from any other effusion into the pleura, without the presence of air.

When, however, a communication exists between one or more bronchial tubes and a defined cavity in the pleura, and when this cavity is of moderate size and is occupied partly by purulent or seropurulent fluid, and partly by air, the physical conditions are very nearly similar to those in which a cavern, resulting from phthisis or pneumonia, is present in the substance of the lung, and is situated close to the surface. The physical signs are therefore similar also. In each case, fistulous, or amphoric respiration, may be observed; in each case metallic ringing or silvery resonance of breathing may exist; and in each case the examiner may be able to detect, either separately, or in combination with the other sounds, the large, thick, sonorous mucous rattle, called gurgling.

One other fluid sound remains to be considered. It is most commonly present in conjunction with disease of the pleura; but it is also, though rather rarely, found in connexion with cavities in the lungs, without any especial affection of the serous membrane.

When, in consequence of a communication with a bronchial tube, or a portion of the lung, the pleu17 * 
ral sac contains a considerable portion of air, and also a small quantity of fluid; or when a phthisical cavity of very large size is similarly circumstanced, there is every now and then heard a very peculiar sort of tinkling noise upon examining the chest. It more nearly resembles the sound caused by shaking a pin in a decanter, than any other with which I am acquainted. This is metallic tinkling ("tintement metallique.") It is, most commonly, heard only at intervals; that is, it may occur once in three, four, or forty inspirations. It rarely, if ever, attends the expiration. It may cease altogether, and reappear after a considerable time. In this respect it seems not unfrequently to be influenced by the position of the patient's body.

It is most probably produced by the continued and rapid reverberation of a delicate sound against the firm and vibrating walls of a large cavity. It is in fact an echo in a small space. The original sound from which the echo proceeds appears most commonly to arise from the bursting of a bubble of air, or from a drop of liquid falling upon the surface of fluid in the bottom of the cavity. But it sometimes seems to be likewise produced by the passage of air over a loose portion of membrane, or thick secretion, situated in a tube at or near the entrance of the cavity. The physical conditions necessary for its production appear to be a large cavity with resonating walls, and containing a large 
portion of air, with a small quantity of fluid. When, as occurs in many, perhaps most, protracted cases of pneumothorax, the cavity in the pleura previously to death becomes in a great measure filled with fluid effusion, and the air is consequently displaced or absorbed, the metallic tinkling disappears, and, unless the fluid so accumulated be evacuated, permanently ceases.

Let the student, however, beware that he is not in this respect, as well as in those before mentioned, deceived by the stomach, which, containing a mixture of fluid and gas, and passing high up beyond its ordinary boundaries, sometimes so far encroaches upon the chest as to give rise to physical signs, many of which exactly resemble those of pneumothorax, and among which a sound very nearly approaching to, if not precisely identical with, metallic tinkling, is occasionally heard.

\section{Auscultation of the Voice and Cough.}

1. In diseases of Air-passages and the Lungs.

The changes produced by disease in the auscultation of the voice in many respects resemble those which have been noticed in reference to the breathing. Thus, it may be generally stated, that whatever increases the power of conducting sound in the thoracic organs, which are connected directly or indirectly with the larynx, also increases the 
resonance of the voice, heard through the parietes, just as it increases the loudness and shrillness of the morbid sounds which accompany the respiration. But as the voice in disease, as well as in health, is formed by the passage of air from the lungs; or by the expiration; and as many, if not the majority, of the morbid sounds which accompany the breathing are produced by the passage of air to the lungs, or by the inspiration, many and important alterations affect the one, which are in no respect applicable to the other. Thus, mucous rattles may be heard throughout the whole chest, and the voice may remain perfectly natural; and the voice may be quite inaudible, and yet the respiratory murmur may continue pure in every part. Sounds also which accompany the respiration may and often do, originate in the pulmonary tissue itself, and exist independently of any sound arising in, or conveyed by, the bronchial tubes; but these tubes, as regards the voice and the cough, are, both in health and in disease, the principal and primary channels by which the sound is conveyed to the parietes.

In the diseases of the Larynx the alterations of the voice which exist in ordinary conversation are also observable upon the application of the ear to the chest, or of the stethoscope to the side of the neck. The only assistance afforded by auscultation, in disease of the larynx, with which I am practically conversant, is, that it enables us to as- 
certain the exact seat of the disease, or of the obstruction which gives rise to the alteration, or to the loss, of the voice. Of the nature of that disease, or of the character of that obstruction, it telleth not. The same acknowledgment was made while treating of the respiration.

As in ordinary Bronchitis the texture and consistence of the lung are unchanged, the voice, for the most part, remains unaltered. But when, in consequence of long-continued bronchial obstruction, the lung becomes considerably congested with blood, or infiltrated with serum, the organ is rendered comparatively solid.-Commensurately with its degree of solidity it is also rendered a better conductor of sound. The voice, therefore, and the cough, are, in the parts so affected, louder than in other parts of the chest. Those parts are ordinarily at the base of the lung posteriorly, as they are generally the most dependent parts, and the fluids, consequently, have a tendency to accumulate therein from gravitation.

When the bronchial tubes are generally, but at the same time slightly and simply dilated, as a result of old bronchial obstructions, the resonance of the voice is often only very slightly, or not perceptibly, increased; as not only are the parietes of the tubes themselves less thick and dense than in the normal condition, but the surrounding pulmonary tissue is often more or less affected with emphy- 
sema; the necessary consequence of which is, that the voice is less perfectly conducted to the parietes than in the natural state of the organ. But when dilatation is partial, and at the same time extensive; when, that is to say, the dilatation affects one, or only a few tubes, in a defined portion of its or of their course; when, in fact, one or more circumscribed cavities are formed by the dilated tubes; the resonance of the voice may be considerably increased over the part in which such dilatation exists; and if it be near to the surface of the lungs, and particularly if it be surrounded by consolidated tissue, the voice may be not merely increased in resonance, but the increase may amount to bronchophony, or even to perfect pectoriloquy. These terms have already been explained and illustrated, while speaking of the natural voice (see p. 141.) It is therefore not necessary here to repeat the account of them. But if the student have any doubt of his capability of discriminating between them, let him turn to that page, and repeat the experiments there recommended to his notice.

In simple emphysema of the lung, the density of the organ is diminished, and its conducting power consequently decreased; the resonance of the voice, therefore, as heard through the parietes of the chest, is proportionally lessened.

When the lung is consolidated from Pneumonia, its conducting power is increased; and if the tubes 
passing through, or in the vicinity of, the consolidated mass, be of tolerable magnitude, as in the upper part, or central portions of the lung, the voice is more easily transmitted, and bronchophony is consequently heard. But if the consolidation exist only at the edges of the lung, removed a considerable distance from any tube of large calibre; or if, though in the neighbourhood of large tubes, those tubes are closed by compression from without, or by secretion from within, the voice is not only not increased in resonance, but it may be less loud than natural; as the tubes, which are the principal conductors of the voice from the larynx to the parietes, do not, under such circumstances, in consequence of the absence of air, vibrate with sufficient power to affect the solid walls of the chest. Thus at the angle between the diaphragm and the ribs, perfect consolidation of the lung may exist without any increase of the natural resonance of the voice. Thus, also, when, as sometimes, though rarely, happens, the tubes are plugged up with plastic secretion, while the lung itself is converted into an airless mass, the voice may be scarcely audible through the parietes.

In persons who, being either constitutionally feeble, or having been debilitated by disease, have been confined for a long time to one position in bed, as in the case of fracture of the old, and in typhoid fevers of all ages, blood accumulates in the 
more dependent parts of the lung, the vessels of which become dilated, and the tissue of which is rendered less elastic, and, at the same time, soft and friable, from the increase of fluid and the comparative deficiency of air. From long continuance of this state of passive congestion, it is not improbable that structural change may sometimes occur, and that a low form of pneumonia may be induced, similar to that, which occurring in the last few days or hours of life, is called by the French pathologists "pneumonie des agonisans," or the pneumonia of the dying. But whether the changes induced in the lung amount in any, or in most, cases to pneumonia, it is certain that the density of the lung is increased, that a certain degree of consolidation is produced, that its power of conducting sound is improved, and that, as a direct consequence the resonance of the voice over the part becomes so much greater than natural as not unfrequently to amount to perfect bronchophony.

The preceding observations are, in the majority of instances, applicable to that condition of the lung which ordinarily exists in pulmonary apoplexy. They are, indeed, perfectly relevant, as regards the state of the lung when blood has not escaped into the pulmonary tissue. But while the passive congestion, arising from debility, always exists in the most dependent parts of the lung, that 
resulting from obstruction, as from diseased heart, or other similar causes, may exist in any part; though it is most frequently found in those portions of the lung which are the farthest removed from the central organ of the circulation.

When the tissue of the lung is broken down, or its cells are filled with effused blood, when the bronchial tubes are not compressed or plugged up, and when the part of the lung affected is favourably situated for the resonance and conduction of the voice, the bronchophony, which is present in consolidation from other causes, will also accompany that resulting from pulmonary apoplexy. But as, in both these respects, the lung is often unfavourably situated; as the part affected is frequently the most distant from the larynx, as well as from the heart; as the tubes are often compressed or obstructed by the effused blood; and as, moreover, pulmonary apoplexy often exists in small detached masses, distributed here and there throughout the lungs; increased resonance of the voice is not so common in this affection as in the consolidation of the lung from other causes.

When, in consequence of the destructive progress of unorganizable pneumonia, a cavity is formed in the lung, the state of the voice varies according to the seat of the cavity, and the condition of the surrounding textures. If it be at the thin edge of the lung, if the tubes leading to it be small, and if 
the pulmonary tissue, intervening between it and some one or more large tubes, be not consolidated, the voice may be scarcely increased in resonance. If, on the contrary, it be in the upper part of the lung, or near large tubes; or if it be close to the surface of the chest, or surrounded by consolidated pulmonary tissue, then either bronchophony, or imperfect or even perfect pectoriloquy, will be present, according as the situation and the condition of the diseased parts are more or less favourable to the transmission of the voice. Pectoriloquy by no means necessarily exists under such circumstances. There may be a cavity in the lung without pectoriloquy; as pectoriloquy may be present without a cavity.

When, instead of being softened and broken down, and by expectoration reduced to a cavity, a portion of lung affected with pneumonia becomes dense, firm, and contracted, and by adhesions is rendered almost one with the parietes; and when into this portion of the lung there enter large bronchial tubes, whether the large size of the tube be natural or the result of disease, the voice is often rendered exceedingly loud indeed. When, in addition to this state of parts, the disease is situated near to the larynx, the whole of the circumstances contribute to increase the vocal vibration. The resonance not merely equals that existing when the patient speaks into the tube, or when the tube is 
placed upon the larynx; it is not simply pectoriloquy; but the voice becomes so shrill and jarring as to be actually painful to the examiner.

This may be one of the comparatively few cases in which, as it appears to myself, consonance, properly so called, may be presumed to exist; in which the voice, originating in the larynx, is not merely conducted by a large tube into a cavity, and from it to the parietes; but from a similarity in the celerity and extent of their vibrations-in other words, from the consonance of the solid walls of the cavity - with those of the larynx, those vibrations are increased in power, and consequently impinge upon the ear with even greater force than those proceeding directly either from the larynx, or from the mouth itself.

In Malignant disease of the lung, the abnormal deposit is sometimes, though comparatively rarely, diffused through a portion of the pulmonary tissue, without materially compressing the bronchial tubes. Under such circumstances (of which I have only seen a few examples,) the voice is rendered more loud than natural, commensurately with the amount of consolidation of the part, its permeation by large bronchial tubes, and its vicinity to the larynx. At other times the malignant mass encroaches upon the lung, and instead of invading its texture, pushes it aside, and with it the bronchial tubes by which it is traversed. Under such circumstances there 
exists a solid mass, containing no conveying pipe from the larynx, or, in other words, no prime conductor; the resonance of the voice, therefore, is not only not increased, but that naturally existing in the part affected is diminished, and sometimes even lost. According to its position and arrangement, and to its disposition in the massive or tubercular or infiltrated form, malignant disease of the lung then may be accompanied with increased or with decreased resonance of the voice, or the voice may be entirely absent in the part affected.

In the earlier stages of Phthisis the degree of resonance of the voice depends upon the amount of tubercular deposit, the consequent density of the lung, and its resulting conducting power. Even in very early stages, when few tubercles exist, or perhaps, to speak more correctly, when the tubercles are accompanied with little or no surrounding pneumonic consolidation, and when little or no pleuritic deposition is present, there may be sometimes heard a slight increase of vocal resonance in the acromial or infra-clavicular regions of one side, over that of the corresponding regions of the other side. The student, however, must be here especially vigilant against error. He must recollect that in many, and especially in thin persons, the voice is naturally very resonant below the clavicles and above the spine of the scapulæ, and that it is sometimes slightly more resonant upon the right 
than upon the left side. He should, therefore, never form an opinion in consequence of the resonance of the voice in the superior parts of the chest on one side, without comparing it with that of the other. He should also never forget that greater resonance may exist upon one side than upon the other, without disease; and that this increase, when it is independent of disease, usually exists upon the right side. Unless, therefore, the increased resonance of the voice be confined to one side of the chest; unless it be combined with other physical signs, or with obvious constitutional symptoms; or unless it be of so decisive a character as not to be satisfactorily explained by natural conformation, it should not be regarded as an evidence of disease. (Though pectoriloquy in its perfect form be not very common in even advanced phthisis, and though scarcely any physical sign be less determinate in its presumed indications; yet no sign is supposed to be discovered by the inexperienced student more frequently than pectoriloquy, which is at least as often mistaken as it is misinterpreted.)

As the consolidation of the lung increases, whether by the greater number of tubercles, or by attendant pneumonia, the voice becomes more and more resonant, and bronchophony, or imperfect pectoriloquy, may be observed in the part affected. The farther that part is from the larynx, the less likely is the bronchophony to be the result of na$18^{*}$ 
tural conformation; the more distant, therefore, it is from the larynx and the larger divisions of the trachea, the more probably does it result from disease; and therefore the more important does it become as an indication of disease, when discovered above the scapula, than on its inner edge, or when near to the acromial process of that bone than when close to the sternum.

When, in the progress of the complaint, cavities are formed in the lung; as those cavities are usually - near to the apex of the lungs, and therefore not far removed from the larynx; as they are almost universally surrounded by consolidated pulmonary tissue, which is adherent to the costal pleura, and which therefore becomes one firm mass connected with the parietes; and as, moreover, these cavities are generally near to the surface of the lung, the whole arrangement of the parts is most favourably circumstanced for the conduction of the voice. Pectoriloquy, perfect or imperfect, is accordingly a very frequent sign of a tubercular cavity.

But though a frequent, is pectoriloquy a certain sign of a cavity? As it has been already stated, it is not. But in a tubercular subject, one evidently suffering from advanced phthisis, may it not be so regarded? No! Well, but if tubular breathing, and contraction, or flattening of the part, exist at the same time, is not pectoriloquy a proof of a cavity? No! not even then!! All this, and much 
more, may exist, and yet the presence of a cavity in the lung cannot be with certainty predicted, even though the part affected be the apex of the organ. Even then it is merely a question of probability, as all these signs may arise from a consolidated and contracted lung, combined with, or even without, dilated tubes. Such a condition of the organ, as has been already stated in reference to pneumonia, may not merely give rise to perfect pectoriloquy, but may render the voice more loud, shrill, and ringing, than when the patient speaks into the trumpet-shaped end of the cylinder. The truth is, that pectoriloquy, that sign which was formerly regarded, and is still esteemed by some, who are not practically conversant with auscultation and with morbid anatomy, as the especial and sure sign of a cavity, is now well known, by those who are familiarly acquainted with the revelations of the inspection-room, to be, as has been before stated, among the most uncertain of the physical signs afforded by auscultation.

When a cavity in the lung is of very large size, the voice, like the breathing, sometimes possesses a peculiar ringing or metallic resonance, in consequence of the reverberation of the sound against its solid walls.

The observations made in reference to the voice are also almost literally applicable to cough. The only remark, indeed, that it appears necessary to 
make, in reference to the resonance of the cough, in contradistinction to that of the voice, is, that the former can sometimes be heard when the latter is inaudible, and that the act of coughing sometimes causes the voice to be heard in a part in which it had been previously inaudible. Thus, in the signs afforded by the breathing, as well as by the voice, a tube may be obstructed by thick mucus, or other secretion, which, by impeding the passage of air into the diseased part, may prevent those signs appearing which depend upon the respiration, and may at least greatly modify those which are connected with the voice. Under such. circumstances, if the patient be desired to cough frequently and forcibly, the obstruction is often removed, and the signs are developed, if they had not been previously observed, or reappear if they had temporarily ceased. It occasionally happens, also, that the cough is resonant when the voice remains unaffected, or is affected only in a slight degree. Upon the cause of this peculiarity it is not always easy to decide; but as it is not removed by the repeated effort of coughing, it appears not to be dependent upon the cause previously referred to:

8. Auscultation of the Voice in Disease of the Pleura.

When a small amount of effusion is present in the pleura, whether that effusion be solid or fluid, 
or whether it be the result of inflammation, or of obstruction, and when that effusion is thinly spread over the surface of the lung, the spongy tissue is compressed commensurately with the amount of that effusion. The compressed lung, and the effusion, being singly, as well as in combination, better conductors of sound than the pulmonary tissue in its normal condition, the sound of the voice is then conveyed more perfectly and distinctly than in the natural condition to the parietes of the chest.

But it, moreover, frequently happens, under such circumstances, that the voice is not only rendered increasingly loud, but that it is curiously altered in tone and quality. It sometimes becomes remarkably shrill, trembling, or bleating. The tone, when in its perfect form, is supposed to resemble that of the bleating of a goat; it is therefore called aegophony, and the voice is said to be agophonic. At other times the voice so conducted has a peculiar nasal twang, very similar to that of the fantoccini. It is hence appropriately termed Punch's voice ("voix de polichinelle.") On other occasions the voice resembles that of a person speaking through a trumpet.

The causes of these peculiarities of the voice are not very evident. They are, however, supposed to be produced by the trembling vibrations of partially compressed tubes, modified by their pas- 
sage through a thin layer of fluid. Upon what the differences of the rgophonic sound depend, seems at present to be quite inexplicable; nor, unless the actual condition of the parts at the time could be clearly ascertained, does it appear probable that they will be ever clearly and demonstratively explained. The whole of these sounds are by some called agophony, or considered as modifications of agophony. If the whole be considered as coming under that designation, ægophony is of very frequent occurrence; but pure ægophony, the true goat-bleating noise, is exceedingly-rare. It may be perhaps regarded as among the most uncommon of auscultatory signs. In more than twenty-five years of hospital practice it has very rarely occurred under my observation.

Sometimes the voice possesses a tone and quality not precisely similar to, or rather not exactly amounting to, either of the characters which have been just mentioned. It is increased in power; it is louder than natural; but it is not simply bronchophonic; it has a different tone from the natural voice; it possesses greater shrillness, but the shrillness does not possess exactly the qualities of either of the varieties of ægophony. It partakes of the character of both, but does not actually resemble either of the two. The tone of the voice under such circumstances has been not inappropriately termed aggophonic bronchophony. The condition of 
the organs of respiration, with which this character of the voice is associated, is uncertain. It is not improbable that it may be connected with slight consolidation, together with effusion.

Is, then, agophony a certain sign of effusion? Far from it. So far, indeed, is this from being true, that as ægophony of the pure bleating kind is among the rarest, so ægophony of every sort or kind is certainly among the most uncertain of the physical signs, considered as a diagnostic of effusion. Agophony, ordinarily so called, may, it is certain, exist without effusion into the pleura, and effusion into the pleura it is equally certain may be present without ægophony. On no occasion, therefore, should ægophony be regarded as positively indicative of effusion.

The situations in which it is most commonly heard, are the infra-scapular, and the inter-scapular regions. But in these regions, ægophony is in some persons said to exist independently of disease. Hence arises the very important practical rule, that ægophony should never be regarded as of any importance, and particularly of any value as diagnostic of effusion upon one side of the chest, unless it have been previously ascertained whether or no it is also present upon the other side. It is, indeed; true that fluid may be present, and give rise to ægophony upon both sides. This in active disease is not very frequent. But when it occurs, its pre- 
sence must be determined or confirmed by the employment of other means, than by the auscultation of the voice.

As the quantity of fluid in the pleura increases, the ægophonic character of the voice gradually disappears. Bronchophony may for a time supply its place; but as the fluid accumulates, the lung, and with it the bronchial tubes, are removed farther and farther from the parietes; so that the resonance of the voice gradually decreases till it becomes almost perfectly inaudible.

An exception of course exists, in this respect, in reference to the inter-scapular region, in which are situated the large bronchial tubes at the root of the lung; as the greater the amount of fluid, the more closely are they commonly pressed against the parietes. In this region, therefore, the voice is ordinarily even more distinct and loud than in the natural condition, at the time that it is scarcely audible in other parts of the same side of the chest. The exceptions, which were referred to while speaking of bronchial respiration as the result of effusion, and of its disappearance under ordinary circumstances, when the fluid accumulated beyond a certain extent, are likewise applicable to the voice.

The curious exception to the general rule of tubular breathing and vocal resonance disappearing, except in the inter-scapular region, when 
fluid exists in large quantity in the pleura; the fact, I mean, of both these sounds being sometimes heard all over the side of the chest, when a large amount of fluid has been proved to be present in the pleura, is sometimes observable in reference to the voice, and not to the breathing. In a case, for example, in which seventy-two ounces of fluid were drawn off by paracentesis, the voice was shrill and resonant all over the side of the chest, though the tubular breathing was only heard in the interscapular region.

Another curious fact in reference to effusion may be again here referred to. It sometimes happens, as has been before stated, that the voice appears more shrill to the ear than in the natural condition, while the vibration of the parietes, as appreciated by the hand, is less than natural, or even entirely lost. Tactile vibration, in other words, is absent, though the vocal resonance, as appreciated by the ear, is, or at least appears to be, increased; and yet both are communicated by the vibration of the same parietes. The cause of this very remarkable fact, which is now acknowledged by all practical auscultators, and which $I$ have often pointed out to pupils and others, is obscure, as I have already mentioned while treating of Palpation, to which the student is referred for what appears to be a possible explanation.

When, in consequence of a communication ex19 
isting between the pleura and a bronchial tube, a tubercular cavity, or a portion of gangrenous lung, air is present in the serous membrane in considerable quantity, the voice and the cough possess the same ringing or metallic resonance which has been already noticed while speaking of the breathing in pneumothorax. It usually accompanies both the voice and the cough; but it is sometimes observed to attend the one, and not the other. Under such circumstances, it is generally, I believe, the cough which possesses, and the voice which is destitute of, the metallic character. This probably arises from the greater muscular effort exerted, and the consequently greater vibration excited in the air contained in the cavity by the act of coughing, than by that of speaking. The character of the sound itself it is unnecessary to attempt to explain, or to illustrate farther than it has been already done. It should be heard to be properly appreciated. When it has been once heard, it is scarcely possible that it can ever after be mistaken.

\section{Auscultation of the Heart.}

While speaking of the auscultation of the respiratory organs, it was stated that it was necessary for the student to be acquainted with the signs existing in the healthy state, previously to commencing the investigation of the physical effects of disease. This previous knowledge is more espe- 
cially necessary in reference to the heart; as without clear and definite notions of action, and familiarity with the sounds of the organ in health, it will prove utterly futile for him to attempt fairly to appreciate the physical signs of its diseases. A brief, and, as far as practicable, a simple account, will therefore first be given of the auscultatory signs afforded by the heart in the healthy condition, and afterwards will be rnade a short exposition of those which occur when the organ is diseased.

\section{Auscultation of the Heart in Health.}

The physical circumstances which are to be observed in reference to the auscultation of the heart are included under its Impulse-its Rhythm-and its Sounds.

(a.) The Impulse of the Heart.-When the expanded hand is placed upon the left side of the chest below the nipple, or more particularly when the points of the fingers are placed upon the space between the fifth and sixth ribs, a little to the inner side of a line running vertically over the nipple, a gentle and regular pulsation is felt, and when the ear or the stethoscope is applied to the same part, a slight shock is perceived by the examiner. This is the Impulse of the heart, dependent upon the impinging of its apex against the parietes. It is nearly or quite synchronous with the pulse at the 
wrist, and with the systole or contraction of the ventricles. In the healthy adult it occurs from sixty-eight to eighty times in the minute. It has been variously explained at different times, and diverse and even opposite theories have been promulgated for its elucidation. Thus the lengthening of the aorta, and the straightening of the curved arch of the aorta; the recoil of the apex from the compressed blood, from its being situated opposite to the open mouth of the aorta, analogous to the recoil produced by the discharge of a gun; the rounding of the body of the ventricle, and the lengthening of the same, have been at various times, and by different authors, advocated as the cause of the impulse. Some of these alleged. causes may tend to produce the general effect, but it is clear that all of them cannot be correct, or even contribute to the general result. I think that, from examination of the action of the heart in living animals, it is now generally believed, at least in this country, to arise mainly from the apex of the heart striking against the parietes. A tilting forwards is supposed to be produced by the contraction of spiral bundles of fibres, of which the ventricle is principally composed-the fixed point or point d'appui of which is at the base of the heart. This contraction causes the apex to move in the section of a circle, of which the base is the axis or centre, and to thereby strike against the ribs. Hence is supposed chiefly to arise the Impulse. 
The part in which the impulse is felt, in the healthy condition of the organ, varies considerably, according to the position of the body.' While it is erect the impulse is felt about an inch to the inner side of a line running vertically over the nipple. When the individual examined lies upon his back it is decreased in force, and, at the same time, felt about half an inch nearer to the median line of the body. When the trunk is turned directly upon the left side, the impulse is perceived either immediately below, or sometimes considerably to the outer side of, the nipple; while, when the trunk is turned directly upon the right side, it is either not perceptible at all, or only just perceptible between the cartilages of the ribs close to the sternum.

The impulse of the heart, like the pulse at the wrist, is naturally much more frequent in some individuals than in others. It also, quite irrespectively of disease, varies very considerably in character. It is full and powerful in the strong and robust; while, in the weak and delicate, it sometimes amounts to scarcely more than a gentle rising or undulation, the precise situation of which it is occasionally difficult to determine. In the vigorous but calm individual, it is strong, moderately heaving, and diffused; in the weak, nervous; and excitable, it is smart, smacking, and defined, or, as it were, concentrated. When, indeed, the organ itself, and the arteries leading from it, are 19* 
healthy, the impulse of the heart resembles in character and in power, as well as in frequency, the pulse at the wrist.

The precise situation of the impulse may be best defined by the fingers, or by manual examination; though by those who have already become accustomed to the use of the stethoscope, its character and power may generally be best appreciated by: the sensation communicated thereby to the side of the head. In one case, for example, as in a feeble or fatty heart, it may shake the head scarcely at all; in others, as in the irritable heart, it may effect a sharp knocking stroke; while in a third class of cases it may, as in the strong or hypertrophied heart, without knocking, forcibly lift up the head, or communicate to it a heaving sensation.

Here it may be desirable to caution the student against confounding together a sharp or smacking impulse, and a forcible or heaving one. This is particularly necessary; as the former, by those who are little conversant with the matter in hand, is not unfrequently, or is, perhaps, generally, denominated a strong impulse, and is most incorrectly supposed to indicate a thick, powerful heart; whereas it almost always characterizes the very opposite condition of the organ. Let the student ever recollect, that a sharp, smart, smacking impulse, merely indicates an irritable heart, and usually a weak one-like a pulse of the same character; 
whereas it is a deep, heavy, heaving impulse, like a large, full, and incompressible pulse, which accompanies a powerful or hypertrophied heart.

(b.) The Rhythm of the Heart.-If the ear, or the stethoscope, be placed upon the præcordial region, when the heart is acting naturally, a regular succession of sounds, and cessations of the sounds or of intervals, will be noticed by the most casual observer. In the first place will be noticed a sound comparatively long, then a sound comparatively short, and then an interval without any sound; after which, again, will occur the long, then the short sound, and then the interval. The long and short sound, together with the interval, make up the time occupied by one complete circuit of the heart's function; and as these sounds and this interval have been observed constantly to bear a definite relation to each other, the period occupied by them, or the complete circuit of the heart's action, has been divided into corresponding portions of time.

Supposing, then, that the whole period of this time be divided into fifths, - the first sound has been calculated to occupy two-fifths, the second, sound to occupy one fifth, and the interval between the sounds to occupy the remaining two-fifths. The period occupied by the whole corresponds to the time between one stroke of the pulse and 
another. So that if a person's pulse beat eighty times in a minute, there would be eighty long sounds, eighty short sounds, and eighty intervals. Similar relations would exist, supposing that they could be correctly estimated, and that the pulsations were at the same time regular, if there were one hundred, or a hundred and sixty strokes of the pulse in a minute. This regular division of the time occupied by one complete circuit of the heart's function constitutes the Rhythm of the organ.

This may be perhaps termed the external, or obvious rhythm; as there exists, also, an internal rhythm, upon which the external one depends, and of which, therefore, it is the indication.

It has been observed, while watching the uncovered heart of the lower animals, that after the interval, or repose of the heart, the contraction of the appendix of the auricles first occurs, and is immediately followed by the systole of the two ventricles, which contract simultaneously. At the same time occur the first sound, the distention of the aorta and of the pulmonary artery with blood, comparative fulness of the auricles, and the tilting forward of the apex of the heart. Immediately after the systole or contraction of the ventricles, follows the sudden diastole or dilatation of the ventricles. At the same time occur the reaction of the distended aorta and pulmonary artery upon their contained blood, the second sound, and the 
comparative flaccidity of the auricles. Then occurs the pause or interval between the sounds. The ventricles are meanwhile becoming full, but not distended with blood; the appendices of the auricles again contract; a small additional quantity of blood is sent into the ventricles, which are supposed to become thereby distended, and by such distention to be excited again to contract, when the series of actions takes place as before.

Such are, very briefly, the actions, as related by experimentalists, upon which the Rhythm of the heart depends, and such, therefore, is what may be denominated its internal Rhythm.

When the sounds of the heart, and the interval between them, bear the relation to each other which has been mentioned above, the rhythm is said to be natural: when they do not bear that relation to each other which has been mentioned above the rhythm is said to be unnatural, and the action of the heart is said to be irregular. Irregularity of action is not, however, always the same thing as an unnatural rhythm, as, in the case of a slow pulse, the heart may be quite regular, although the state of repose, or the interval between the sounds, may be vastly increased.

When the pause, or interval between the sounds, is longer after some contractions than after others; and when this increased duration of the pause or interval occurs at regular periods, whether it be 
every third, sixth, or sixteenth beat of the heart, the action of the organ is said to be intermittent. But when, though the sounds and intervals bear the same relative proportion to each other, as in the natural condition, one stroke of the heart is observed to be more powerful than the stroke, or than the four, six, or any other number of strokes, which precede and succeed it, the action is said to be unequal. Irregularity of the action of the heart, then, depends upon an alteration in the natural succession of sounds and intervals, and is connected with the Rhythm. Intermittence is the regular recurrence at stated intervals of that irregularity, and is also connected with the Rhythm. Inequality exists when one stroke of the heart is stronger than another, or than several others, and is connected with the Impulse. It is, in fact, an irregularity of the impulse.

Is, then, a natural rhythm indicative of a sound heart, and an unnatural rhythm necessarily characteristic of an unsound one? Both these questions must be answered in the negative.

A heart greatly diseased, so long as the circulation is undisturbed, and the patient remains quiet, mentally as well as bodily, may pulsate with perfect regularity. The rhythm of a merely irritable heart, as that of an hysterical girl, a dyspeptic, a valetudinarian, a hypochondriac, or of a person greatly alarmed or excited, may exhibit all 
sorts of irregularities. The Rhythm, therefore, considered alone, is of little diagnostic importance, as the mere fact of making an examination for the purpose of ascertaining its character, may, in a nervous subject, alter it altogether.

(c.) The sounds of the heart.-The natural sounds of the heart, the regular recurrence of which has been already referred to as constituting the Rhythm, may be fairly represented by the following syllables, too-to-too-to. The origin of these sounds has been the source of much discussion, and the object of a great variety of experiments. The student may see an account of them in most systematic treatises on diseases of the heart.

It does not consist with the design of this work to enter into a critical examination of this or of similar questions. It may be simply observed, that the opinions hereafter expressed, are those which appear, and ever have appeared, to the author to be the most probable. It is entirely opposed to the object I have in hand to engage in any arguments in support of those opinions, or conjecturally to answer any objections which may be possibly raised against them.

I proceed, then, at once to inquire what is the efficient cause of the two sounds of the heart? In answering this question it will be convenient first to consider the origin of the second sound, which 
is the more simple, admits of less dispute, and is now generally believed to be dependent upon an obvious and acknowledged cause; and subsequently to consider the origin of the first sound, concerning which there still exists much diversity of opinion.

The origin of the second sound.-If the cock of a leaden pipe, which is not firmly fixed to its solid support, be turned, and a full stream of water be allowed to pass through the aperture which that cock controls, little agitation of the pipe is induced by the mere passage of the fluid. If, however, while the water is passing with rapidity and force, the cock be suddenly turned, and the passage of the fluid through the aperture be in consequence suddenly arrested, a very considerable agitation of the pipe will be immediately perceived, and a corresponding noise will be heard. This agitation, and this noise, are clearly the consequence of the vibrations excited in the fluid by the sudden arrest of its onward progress; which vibrations are communicated to the solid walls of the pipe, and thus become obvious to the eye, as well as to the ear. Another illustration may be taken from what is seen to occur at the closure of a lock on a river. So long as the water continues to flow through the constantly narrowing opening between the two arms of the lock, there is no agitation in the back water. But immediately the two arms meet, a vio- 
lent shaking is observed in the wood of the lock itself, and in the water which is behind it. This is evidently produced by the sudden stoppage of the onward progress of the stream.

Now the circumstances attending the second sound of the heart are precisely similar to those which are observed in the pipe and in the lock; excepting that the former are connected with living and elastic tissues, and the latter occur in connexion with comparatively inelastic materials and with dead matter. The blood is, by the contraction of the ventricles, propelled into the aorta and pulmonary artery, and these vessels become thereby distended. When the pressure from behind is removed by the sudden diastole of the ventricles, the large vessels react (whether by actual contraction, or by mere elasticity, does not affect the present question,) powerfully react, upon the fluid which distends them. The blood is forced back towards the ventricles. Some of the fluid, it is almost certain, must re-enter the cavities. But its retrograde motion is suddenly arrested by the flapping back of the sigmoid valves. These flood-gates suddenly stop the backward progress of the blood; and this sudden stoppage of the forcible current, as in the case of the pipe and cock, or in that of the lock, produces a violent agitation in the particles of the blood, which is communicated to, and induces powerful vibrations of, the coats of the vessel, and con- 
sequently of the parietes of the chest. Hence arises the second sound of the heart.

Origin of the first sound.-The first sound of the heart appears mainly to arise from causes which are, mutatis mutandis, almost precisely similar to those which produce the second sound. If the ventricles be supposed to represent the aorta and pulmonary artery, the auriculo-ventricular to replace the sigmoid valves, and positive be put for probable contraction, or elasticity, then one description might be almost literally applied to both. When the contraction of the ventricles commences, the passage to the auricle, it must be recollected, is at least partially open. There is a space open for the blood to pass into the auricles as well as into the large arteries. Some blood, it may be pretty confidently asserted, at first does actually pass back into the auricles; though the question, whether a small quantity does or does not pass, does not affect the theory of the sound. The blood, strongly compressed by the forcible contraction of the ventricles, has at least a tendency to pass into the auricles as well as into the aorta and the pulmonary artery; but the flood-gates at the entrance shut the opening; its backward progress is suddenly stopped by the auriculo-ventricular valves. This sudden stoppage of the blood, as in the case of the lock upon a stream, produces a violent agitation in the fluid, which is communicated 
to the body of the ventricles, and thence to the parietes of the chest. Hence mainly arises the first sound of the heart.

The difference in the tone, and in the duration of the two sounds, appears to be sufficiently explained by the different constitutions of the parts engaged in producing and conducting them. The size of the auriculo-ventricular valves, and the thickness of the ventricles, compared with that of the valves, and of the coats of the vessels; and the irregularity and great inequality of the surface, the musculi pectinati, the carneæ columnæ, and tendinous cords of the ventricles, compared with the smooth lining of the two great arteries, appear amply sufficient to account for the difference.

I have said, upon this cause mainly depends the first sound of the heart; and I feel quite positive that in a practical point this should be esteemed the really efficient cause. At the same time I do not presume to deny that the stroke of the ventricle against the parietes of the chest may produce some sound; or that there is such a thing as a muscular sound of the heart (bruit musculaire, which may possibly contribute to the general effect. But I feel assured that these two, and all other presumed causes of the first sound of the heart, except that mentioned above, may, in a pathological point of view, be practically disregarded.

Natural range of the Heart's sounds.-It is ex- 
ceedingly difficult indeed to define the natural limits of the sounds of the heart; as in scarcely any two individuals are they precisely similar. There are so many circumstances entirely extraneous to the heart itself, which tend to increase or diminish the area over which they are heard, that scarcely any defined line can be strictly said to include it. The sounds, for example, are heard more extensively in a thin than in a fat person; in an individual of diminutive size, than in one of large bulk; and in a person of a naturally irritable disposition, than in one of calm temperament. In some excitable females, indeed, and in males who approach them in character and configuration, the sounds of the heart may be heard over almost the whole thorax; while in others of large frame, with a deep chest, and much adipose tissue below the integuments, of a quiet and unexcitable disposition, they are sometimes scarcely audible in the præcordial region itself. From the mere extent, then, over which the sounds can be heard, considered per se, no conclusion can be properly derived, as to the heart being healthy or diseased.

Irrespectively also of any disease in the organ itself, there are many complaints which cause the sounds of the heart to be heard in some directions more distinctly than in others. Thus, for example, a consolidated lung may cause them to be heard in certain situations, in which they would not be 
otherwise audible. Hence it happens, that the fact of the sounds of the heart being heard more distinctly than usual in one or both infra-clavicular regions, while they are not more loud than natural in other parts of the chest, becomes one of the physical signs of even early phthisis.

An infusion of fluid or of gas into the pleura, a morbid growth, and even a distended stomach, may so displace the heart as to prevent its sounds being heard in the normal situation, while they become audible in situations which are abnormal, as on the right of the sternum, \&c. When the lungs are extensively affected with emphysema, the heart may be so far separated from the parietes, by those bad conductors of sound, emphysematous lungs, as to be almost or entirely inaudible in every part, excepting at the scrobiculus cordis.

Perhaps it would be on the whole most correct, as it would certainly be most intelligible, to say that the heart, when free from disease, in the perfectly natural condition of the organ, and of the surrounding parts, is heard only over the procordial region. By the præcordial region is here, and throughout this work, intended, not a part included in any imaginary or arbitrary line, but the space on the anterior surface of the chest which covers the heart, and the origin of the great vessels. It may, in addition, be stated that while the first sound is heard most distinctly over the junc- 
tion of the fifth left rib with its cartilage, the second sound is most clearly audible on the sternum, near the third intercostal space of the left side.

But these sounds, it has been already mentioned, very often indeed extend beyond these limits, without any appreciable disease in the heart, or in other thoracic organs. What, then, is the usual order of such extension? What are the regions in which the sounds are, under ordinary circumstances, successively audible? To answer these questions with exactitude, the sounds should first be considered separately, and afterwards in connection with each other. The first sound, then, will generally be found, in the first place, to be audible over the left mammary, then over the inferior sternal region, to the scrobiculus cordis, and then over the superior sternal region; while the second sound, if separately regarded, may generally first be heard over the superior sternal, then in the right, and afterwards in the left infra-clavicular region. After extension to the regions just named, the two sounds will commonly be heard with varying distinctness in the other regions, nearly in the following order: the right mammary, the left lateral, and left axillary, -the interscapular, the left, and right scapular, the right lateral, and right axillary,- - the left and right acromial, the left subscapular, and hypochondriac, and the right subscapular, and hypochondriac regions. 
The sounds of the heart are somewhat modified, though considerably less than the impulse, by the position of the body. Thus they are heard more distinctly in the erect position, when the organ more nearly approaches the surface, than in the recumbent posture, in which the heart is farther removed from the surface. They are also heard less distinctly than usual upon the left side of the chest, when the body is turned to the right side, and vice versâ.

\section{Auscultation of the Heart in disease.}

\section{The Impulse in disease.}

When the pulse at the wrist is materially affected by disease of whatever kind, a corresponding alteration will be observed in the Impulse of the heart, unless some mechanical impediment exist to the course of the blood. If, in fevers and the phlegmasiæ, the pulse be strong and vigorous, the Impulse is so likewise; if the pulse be feeble and depressed, so is the Impulse; when the pulse is contracted and vibrating, as after hemorrhage, or in anæmia from other causes, as chlorosis, abstinence, \&c., the Impulse of the heart partakes of the same character.

When hypertrophy exists - when, in other words, the parietes of the ventricles, or of the auricles, but of the left ventricle more especially, are thickened-whether that hypertrophy arises from ob- 
struction existing in the valves, or in the aorta, or from other causes,-the Impulse is powerful and heaving. When, together with thickening of the parietes, there also exists dilatation of the cavities, the Impulse is more extensively diffused, as well as more powerful and heaving, than natural, and may often be felt over nearly the whole of the præcordial region.

When the parietes of the ventricle, instead of being thick and powerful, are thin and weak; or when the muscular substance is pale, flabby, or loaded with fat, the Impulse is feeble, but is sometimes also smart, or, as it were, concentrated. When, together with this weakness, thinness, flabbiness, or accumulation of fat, the cavities of the ventricles are also dilated, the Impulse, though sometimes so feeble as to amount to scarcely more than a gentle tremor, is observed over an extent of surface larger than that in which it is ordinarily felt.

When the individual examined is nervous and excitable, is labouring under chlorosis, or is anæmic from other causes, the impulse is often exceedingly sharp and smart. This kind of beat is constantly mistaken, by the uninitiated, for a powerful impulse. The patient is said to have a strong impulse, and is supposed on that account to labour under hypertrophy of the heart. This is far from being true. It is often, perhaps, indeed, generally, 
the very reverse of the truth. The impulse of hypertrophy is full, strong, heaving, and sometimes diffused; the impulse of chlorosis, anæmia, and irritability, is sharp, smart, local, and, as it were, concentrated. The latter appears to proceed from a quick stroke of the apex of the heart against the parietes, like a smart smack of the points of the fingers; while the former seems to be a forcible elevation of the parietes of the chest, by the body of the ventricle, as if the hand itself were applied within, and pushed them forwards.

When obstruction exists in the pulmonary artery, in the mitral valve, or, indeed, in any portion of the heart, or of the large vessels, which is anterior, in the course of the circulation, to the tricuspid valve, the impulse is generally more or less perceptibly increased in the scrobiculus cordis. This arises from the continued distension of the right ventricle, which results from such obstruction, inducing increased energy of contraction, and thus leading to hypertrophy of the parietes, and to dilatation of the cavity of the ventricle.

When, in consequence of great and long-continued obstruction of the mitral valve, or of such widening of the left auriculo-ventricular opening as renders the valve inefficient, or incapable of preventing regurgitation, the blood returns in part through the opening into the auricle, the whole heart, with the exception of the left ventricle it- 
self, becomes dilated and enlarged. Under these circumstances, in addition to the impulse felt at the scrobiculus cordis, dependent upon enlargement of the right ventricle, there is frequently a perceptible impulse between the cartilages of the third and fourth, or even of the second and third ribs of the left side. This superior impulse is not always exactly synchronous with the impulse below the nipple, and at the scrobiculus cordis. When it is synchronous with that impulse, it probably arises from simple regurgitation through the mitral valve. The blood is then propelled back again into the auricle, and by such propulsion may produce the unusual impulse observed in the situation specified. When it is not exactly synchronous, but appears immediately to precede the infra-mammillary impulse, it seems most probably to proceed from the muscular action of the auricle itself, hypertrophied by its constantly increased energetic action.

When, from whatever cause arising, obstruction exists in the aortic valves, or in the ascending or arch of the aorta, increased effort is required to propel the blood to the distant parts of the body. If the person be healthy and strong, increased nutrition results from this increased effort. The consequence of this increased nutrition is thickening of the walls of the left ventricle, or hypertrophy; and the ordinary attendant of hypertrophy is a 
powerful impulse of the heart. Thus an increased impulse very generally accompanies obstructive disease of the aortic valves or of the aorta, and becomes the indication of hypertrophy, which, however, it must be recollected, is not itself the disease, but simply the effect of the efforts of the system to overcome the consequences of the disease.

Should; however, the subject of aortic obstruction be feeble in power, and the system be unable to supply the increased nutrition which is necessary to overcome that obstruction, the walls of the left ventricle, instead of being thickened, become thin, and the cavity of the ventricle dilated. Under such circumstances, the impulse, instead of being strong and heaving, becomes feeble and diffused, and sometimes a mere flutter. In some instances, again, the especial pathological causes of which, independently of obstruction, are not always clear, the ventricle becomes both thickened and dilated, and then the impulse is powerful and heaving, as well as widely diffused.

When the aortic valves are defective as well as thickened, or, in other words, when regurgitative as well as obstructive disease exists, this last mentioned condition very generally, but not universally, exists also. It is not, however, confined to such cases; but, in truth, is not unfrequently found after death to accompany comparatively slight obstructive disease of the aortic valves. 
When fluid is effused into the pericardium, as a result either of inflammation, or of obstruction to the current of the circulation through the heart, the impulse varies according to the qnantity of the fluid effused. If the fluid be small or moderate in quantity, the impulse below the nipple is only slightly decreased in power, while a gentle rising is sometimes felt between the cartilages of the second and the fourth ribs. If the fluid be in large quantity, the regularity of the impulse is lost, and its position is often altogether changed. A confused sort of tumbling motion only, at once irregular and unequal is felt here and there over the præcordial region, instead of the natural impulse.

But it may be inquired-does a powerful impulse necessarily indicate hypertrophy? No! Is, then, hypertrophy always accompanied with a strong impulse? Assuredly it is not!

In explanation of the negative answer to the former question, it may be stated that an aneurism of the descending, or even of the left side of the ascending aorta, may push the heart aside, and, by dilating, and producing partial absorption of, the parietes, may communicate a powerful heaving impulse to the part in which the natural impulse is felt.

In explanation of the negative, answer to the second question, it may be observed that, though the heart be powerful, and the parietes of the ven- 
tricles very thick and strong indeed, the action of the heart may be so hampered by the consequences of obstruction in the valves, and its contractions may be so impeded, and so overpowered by fluid. accumulated either within or without its cavities, as to be almost entirely mastered, and to be only just enabled to flutter a little, and thus to relieve itself of a small portion of its load. Such, indeed, is very commonly the condition of the organ, in long-standing disease of the valves, and particularly in that of the mitral valve, when the nervous energy of the individual is considerably reduced; even though the muscular power of the heart, abstractly considered, be much greater than in health. Such is commonly the condition of the impulse in the last few days of the life of persons affected with disease of the valves, accompanied with hypertrophy. If they do not die suddenly, as persons so affected frequently do, it may indeed be considered to be the natural termination of such cases.

A very large and powerful heart, therefore, may be accompanied by a very feeble, irregular, and fluttering impulse, and a very small and feeble heart may be, and usually is, accompanied with a very "smart" and "smacking" impulse.

It has been stated, (but whether it has been proved by inspection after death, or is only surmised, does not appear,) that when adhesion of the 
pericardium exists, without any disease of the heart itself, the situation of the impulse is not changed, as it is in the healthy condition of the organ itself and of its investing membrane, according to the varying position of the body; and that this immobility of the impulse arises from the heart being by such adhesion tied down to the diaphragm. Now, when the pericardium is adherent, there generally, but by no means universally, also exists disease within the heart itself, the consequence of which is the enlargement of the organ. Under such circumstances it is sufficiently obvious that the situation of the impulse is not so likely, as in the state of health, to be altered by any change in the position of the body (by reason of the increased bulk of the heart;) but whether, in simple adhesion of the pericardium, this presumed fixity of the organ really and necessarily occurs, my own experience does not enable me to decide.

In cases of advanced phthisis of the left side, the situation of the impulse is constantly observed, and seen as well as felt, to be as much as an inch, or even an inch and a half higher than natural. This arises, probably, from two causes : first, partly from the stroke of the heart being communicated to the parietes through the consolidated lung; but, secondly and mainly, from the organ being actually drawn upwards by the partial collapse of a cavity 
or cavities, and by the contraction of surrounding pleuritic deposit.

\section{The Rhythm in disease.}

Any moral impression, or any physical impediment, which unnaturally excites the action of the heart, may at the same time disturb its rhythm. Any suddenly supervening emotion of the mind, therefore, whether of joy, fear, or anger, may, in susceptible individuals, almost instantaneously alter. its character. Persons whose temperament is naturally excitable, or those in whom it has become so from disease or anxiety, dyspeptics and valetudinarians, are especially liable to, what is called, nervous palpitation, which is merely an excited action of the heart, accompanied with an unnatural rhythm, and an unequal impulse. The remote and exciting causes, therefore, of an unnatural rhythm are very numerous. Its proximate cause is an interruption of the regular successions of contractions and dilatations of the auricles and ventricles. One cavity contracts before its time, or another is dilated too quickly; the natural series of actions is interrupted; the regular succession is broken.

Such is an unnatural rhythm. What, then, are the organic changes affecting the heart; what are the physical conditions of the organ which principally tend to induce it? In answer to this question, 
it may be replied, that the physical condition which interferes most with the equable flow of blood through the chambers of the heart; that the organic changes which in the greatest degree contribute to the interruption of the circulation through the organ, are most likely to produce an unnatural rhythm.

Among the most frequent causes, therefore, of an unnatural rhythm, are diseases of the valves; in consequence of which, the cavities behind the seat of obstruction become so distended with blood, and the ventricles thereby so hampered and fettered in their action, that they make various irregular efforts and ineffectual struggles to get rid of the load which oppresses them. This irregularity of the action, or unnatural condition of the rhythm of the heart, occurs much more commonly in disease of the mitral valve, than of the other valves. This fact is most probably explained by disease of the mitral valve being more frequently accompanied with regurgitation than that of the other valves.

Yet, even when the aortic valves admit of regurgitation, an unnatural rhythm does not so frequently accompany the complaint, in proportion to their number, as it does the imperfections of the mitral orifice. Upon what this difference depends it is not easy to determine.

Another very frequent cause of an unnatural rhythm is thinness, great dilatation, or weakness 
of the parietes of the ventricles; from which arises a similar incapacity of emptying their cavities as exists in diseases of the valves. From this cause also probably arises the irregular and defective action, with unnatural rhythm, which often coexists with ossification of the coronary arteries; in consequence of which disease, the muscular structure of the heart is insufficiently supplied with blood, and imperfectly nourished.

An unnatural rhythm often arises also from large effusions into the pericardium, which probably induce the irregular action of the heart by preventing the cavities being equably and sufficiently filled with blood.

In all these cases the unnatural rhythm may vary from a slight occasional irregularity to a perfect jumble of sounds, among which one can be scarcely, if at all, distinguished from another.

It is unnecessary, as it would be futile, to attempt to describe the different alterations of the rhythm which are occasionally observed. Their individual peculiarities are for the most part inexplicable; and a detailed description of their varieties would be attended with no advantage to the student.

One, however, is so peculiar as to require notice. This is the treble beat. The heart, on some rare occasions, is found to give rise to three sounds instead of two, as in the healthy condition. It is, indeed, said that the sounds are sometimes quad- 
ruple, but this fact I have myself never had an opportunity of witnessing. The treble beat, or sound, I have heard on many occasions. It has been composed of two short and one long sound, and may be represented thus - to-to-too, to-to-too, to-to-too. The origin of this peculiarity is uncertain; but it appears most probably to be dependent upon a want of synchronism in the contraction of the ventricles, producing two short first sounds instead of one long one; or to be connected with the pericardium, a slight and short rubbing noise arising from which either overpowers a portion of the natural first sound, and causes it to appear double, or occupies a portion of the interval, or period of repose.

\section{The Sounds in disease.}

The sounds of the heart may be unnaturally increased, unnaturally decreased, or altogether abnormal in character; in other words, the natural sounds may be louder or less distinct than in health, or they may be overpowered and concealed, or supplanted by morbid sounds, called murmurs.

(a.) Increase of the Sounds. - It has been already mentioned that the natural sounds of the heart may appear to be increased in resonance from the surrounding lung being consolidated, and becoming thereby a better conductor of the sounds. With such causes of the increased resonance of these 
sounds we have here nothing to do. Our present observations apply to the increase of the sounds dependent solely upon the heart itself.

This condition is chiefly observed when, the valves being healthy, the chambers of the heart are dilated, or the parietes of the ventricles are thinner than natural. The ordinary result of this change appears to be, that, while the flapping action of the valves is free, the contraction and dilatation of the ventricles is more rapid and smart, and that the agitation produced in the fluid which they contain is greater than in the natural condition of the organ. The vibration, therefore, communicated to the surrounding solids, is increased, and, from the thinness of the intervening barrier, is more readily communicated to the surface of the chest than in the state of perfect health.

It is in such cases that what has been called the "back-stroke" is more particularly observed. This is, in truth, nothing more than a loud, clear, and flapping second sound dependent upon the rapid dilatation of a large ventricle, and accompanied with the sensation of a body retiring from the ear.

When the sounds are loud, as well as clear, enlargement of the cavities, as well as thinness of their walls, will generally be found to exist; when the sounds are clear or shrill, without being particularly loud, the parietes are commonly thin, but the cavities are not dilated. 
The particular regions, to which the sounds of the heart are most commonly successively extended, have been previously mentioned, and need not, therefore, be again referred to in this place.

(b.) Decrease of the Sounds.-A diminution of the natural sounds of the heart may arise from a great variety of causes. Anything which interferes with the free action of the heart itself, or impedes the free motion of its valves; anything which interrupts the full and free contraction of the organ, and consequently decreases the easy and rapid backward flapping of the membranous curtains, tends, in an equivalent degree, to diminish the heart's natural sounds. Over-distention of the organ, therefore, or effusion into the pericardium, from whatever cause arising, may produce this effect: stiffness or want of pliancy in the valves themselves may, without giving rise to a murmur, be attended with a similar result. Morbid sounds, or murmurs, arising from one valve, may obscure the natural sounds arising from the others; and pericardial murmurs, and bronchial rattles, may drown them altogether.

It is also observed that an unnatural thickness of the walls, or simple hypertrophy, of the heart, causes the sounds to be less clear and loud than when the organ is perfectly healthy. The sounds, under such circumstances, may be more deep and full, but they are not so sharp and loud. The cause 
of this appears to be two-fold. In the first place, when the organ is thickened, the contraction of the ventricle, though more strong, is less quick and sharp; it produces, therefore, a less rapid, though it may be a more tight, closure of the valves, and consequently excites less agitation in the particles of contained fluid, and less, vibration in the surrounding solids. In the second place, the thicker the parietes of the heart, the greater mass of solid matter is there to be moved by the vibrations, from which the sounds originate; the less perfectly, therefore, are they communicated to the surface of the chest, from which they are actually more distant, in proportion to the thickness of fleshy substance through which they have to pass. Supposing, then, that they were, as originally produced, of equal intensity or resonance to those existing in health, they might still reach the exterior of the chest, by which they are communicated to the ear, with diminished power, in consequence of the greater thickness of muscle through which they have to be conveyed; for nearly the same reason that they are not so distinct and loud in fat, as in thin, persons.

It may be stated to be consistent with general experience, that a thick heart is accompanied with a powerful, heaving impulse, and with feeble or dull sounds; that a thin heart is accompanied with a smart, and smacking, but not strong, impulse, 
and with loud, or sharp sounds; that a dilated heart is accompanied with a weak but diffused impulse, and with loud and extensively audible sounds; and that a heart which is both dilated and hypertrophied is accompanied with a powerful and widely diffused impulse, and also with sounds which are loud and heard over a large space. These observations have reference, of course, to the several specified conditions of the heart, independently of valvular obstruction, or inordinate distention of the cavities.

(c.) Abnormal Sounds or Murmurs.-The gentle current of a quiet stream, with a level bottom and even banks, passes on with but little ripple, and without noise. If, however, a large stone, or any other resisting body, be thrown into the stream, and, by projecting upwards from the bottom, cause considerable obstruction to the free passage of the water, various little eddies and undulations are observed upon the surface; and if the fall be sufficiently great, or if, in other words, the current be sufficiently strong and rapid, a noise is produced by the agitation of the water thence arising: the quiet "meandering stream" becomes "a babbling brook." If, again, instead of a large stone being thrown into the centre of the stream, a portion of its bank fall in, and be sufficiently resistant to constitute a decided obstruction to the free passage of the water, a similar ruffling and eddying of the surface will 
ensue, and a similar noise will, under similar circumstances, arise from the agitation of the water. But if the mass be so large, and the obstruction so great, as to dam up the water towards its source, and thus to impede the force, and diminish the rapidity of the current, little or no noise will arise; or it will not arise until the back water has become gradually accommodated to the impediment to its downward progress, and the current has resumed its former strength and celerity.

These observations in reference to a stream of water may not be, in every respect, strictly speaking, illustrative of obstructions to the flow of blood through the heart and great vessels; but they are, I believe, in the main applicable to them, and they will, I trust, contribute to render the succeeding remarks both intelligible and easily available at the bed-side.

When the healthy blood passes through the healthy heart in a quiet stream, no noise results from its direct transmission. The valves of the large arteries are appressed to the sides of the vessels, and those between the auricles and ventricles leave a space sufficient for the free passage of the blood. No obstruction exists; no inordinate agitation or eddying of the fluid is produced; and no noise results from the transit.

When, however, the valves become thickened by inflammation, as in endocarditis, they lose their 
pliancy and flexibility; they do not set close to the walls of the vessels, and do not leave a free passage for the blood: when their surfaces are covered, partially or entirely, with plastic lymph, or their free edges are studded with minute granules or vegetations; when they are puckered, wrinkled, stiffened, and corrugated, from the remains of old disease imperfectly repaired; or when they are rendered unyielding and uneven by the deposition of atheromatous or ossific matter below the lining membrane; when, indeed, any mechanical obstruction exists to the free passage of the blood through the apertures which are guarded by the valves, an unusual agitation is excited among the particles of the blood, which, if the rapidity of the current be sufficiently great, gives rise to a morbid sound, or "murmur." Of this character, and of similar origin, is the vast majority, at least, of morbid sounds or murmurs arising within the heart and large vessels. Let it, then, be recollected, that the natural sounds of the heart are produced by the flapping back of the valves, preventing the retrograde movement or regurgitation of the blood, and that the morbid sounds, or "murmurs," of the heart are produced, at least generally, by obstruction to the passage of the blood through the valves.

The morbid character of the sounds may amount merely to an unusual harshness, and increased du- 
ration of the natural sounds, as sometimes occurs in the very early stages of rheumatic endocarditis; when, simple as it may appear, this harshness becomes a most important indication of future mischief, and of more obvious signs of the malady too soon to make their appearance.

It may be only a slight puff or whizzing noise, very accurately resembling that produced by the use of a pair of bellows-hence called "bellows murmur," ("bruit de soufflet;") or it may be like that caused by rasping or filing wood-"rasping murmur," ("bruit de râpe;") or like that arising from sawing wood-"sawing murmur," ("bruit de scie;") or, finally, it may approach to the tone of certain musical instruments, as of a bass viol, a bassoon, or oboe-_" musical sound or bruit.":;

Are, then, these different sounds, it may be asked, characteristic of different states of disease, or are they all merely indicative of obstruction to the progress of the blood? Is the harshness of one sound

* Of the direct cause of the musical tone in certain diseases of the heart my own experience does not enable me to speak with certainty; as in the four or five cases of the kind which I have had an opportunity of examining after death, it has rather curiously happened that two conditions have existed, either of which might by possibility have given rise to it, or both of which might have contributed or have been accessory to its production. These two conditions have been disease of the mitral valve, and especially of the tendinous cords, and cellular adhesion of the pericardium to the pleura costalis. I believe, however, that the sound really arises from the increased vibration of the tendinous cords, or other parts of some of the valves, rendered tense by disease. 
dependent upon a rigid obstructing medium, and the softness of another sound dependent upon a pliable obstructing medium? Does the nature of the deposit materially affect the character of the murmur? Is it harsh and saw-like, with a bony deposit, and soft and bellows-like, with an albuminous deposit? These questions have been answered (it is presumed conjecturally) in the affirmative. My own individual experience necessitates a direct negative. It may, indeed, be pretty confidently asserted that the truth is the direct reverse, and that no such necessary connexion, as à priori might be deemed at least probable, is found in nature; but that, on the contrary, soft murmurs coexist with hard deposits, and that harsh murmurs arise from soft deposits; that nothing can be determined as to the quality of the obstructing cause, from the character of the sound; as weekly experience demonstrates that a very soft murmur may depend upon a rough bony.valve, and that a harsh grating murmur may arise from soft vegetations, or have its origin in even a simple ulcerated opening through a valve.

But is a murmur indicative of valvular disease capable of being referred to any particular valve, or does it merely imply obstruction somewhere? Are there any signs by which the disease of one valve may be distinguished from the disease of another valve? If such signs really exist, what are 
they? In reply to the first question, it may be stated that certain peculiarities do, for the most part, accompany the disease of particular valves. A brief sketch of the anatomical relations of the parts concerned will be requisite, previous to giving a satisfactory answer to the second and third questions.

The valves of the pulmonary artery are situated almost exactly opposite the junction of the cartilage of the third rib with the left of the sternum. The vessel passes forwards and to the left, but is soon directed backwards to be divided into its right and left branches.

The aorta is at its origin partly covered by the pulmonary artery. One perpendicular section would divide part of the valves of both. But the aortic valves, taken as a whole, are about half an inch to the right of, and about the same distance lower down than, those of the pulmonary artery. The aorta, emerging from behind, passes in a different direction from the pulmonary artery. It is directed upwards, forwards, and to the right, till it arrives near to the junction of the cartilage of the second rib with the right side of the sternum; when it crosses behind that bone to form the arch, and then dips downwards and backwards, and becomes the descending aorta.

The mitral valve is situated nearly an inch below those of the aorta, and extends considerably more 
to the left side. It is situated about opposite the space between the cartilages of the fourth and fifth ribs of the left side. The body of the left ventricle, to which it is attached, rests posteriorly upon the spine, the contents of the posterior mediastinum alone intervening between them.

The tricuspid valve is considerably to the right of, and anterior to, the mitral, and it is for the most part covered by the sternum.

Now when these anatomical relations of the different valves are remembered, and when it is also recollected that the morbid sounds or murmurs originate in the circulating blood, and that the vibrations therein excited are conveyed along its current, and are communicated to the solids by which it is surrounded, it will be easily understood, that the murmurs resulting from disease of the different valves, usually occur, or are most distinctly heard, in different parts of the chest.

It may be then stated, as consistent with theory, as well as with experience, that the murmurs resulting from disease of, or from obstruction in, the pulmonary valves, are heard most distinctly opposite to the cartilage of the third rib, or to the interspace between the second and third ribs. It will be also understood why they appear to be very superficial, to pass a short distance to the left, and then abruptly to terminate, or to diminish very considerably in their intensity. 
It may here be observed that murmurs arising from disease of the pulmonary valves are comparatively very rare; and that with the exception of the still doubtful and problematical site of anæmic murmurs, to be afterwards noticed, the pulmonary valves may, in chronic affections, be ordinarily excluded from consideration in arriving at the diagnosis of the origin of morbid sounds.

Diseases of the aortic valves are most commonly accompanied with a sound, which appears to be less superficial than that arising from the pulmonary valves. It is most distinctly heard over the sternum, near to its junction with the third rib of the left side, or the space between the third and fourth ribs. It is heard, though with gradually - decreasing intensity, in the course of the aorta, upwards, and to the right; it often extends as high, or nearly as high, as the clavicle, and sometimes along the top of the sternum.

The murmurs dependent upon disease of the mitral valve are ordinarily most distinctly heard opposite the junction of the cartilages with the fourth and fifth ribs, or immediately below the nipple. They are not so plainly conveyed in the course of the aorta as those of the aortic valves; they are commonly audible in the axilla, and are very frequently heard on the left of the spine opposite to the sixth, seventh, and eighth dorsal vertebræ, or thereabouts; for it is scarcely possible, 
excepting in very thin persons, exactly to mark the particular vertebræ in the dorsal region.

Murmurs, resulting from disease or imperfection of the tricuspid valve, are usually loudest over the central and lower part of the sternum. They are frequently audible at the scrobiculus cordis, but are not conducted in the course of the aorta, nor are they, I believe, heard in the back. But disease of the tricuspid valve, independent of more extensive mischief in the mitral or the aortic valves, is so rare, that it cannot perhaps be positively stated what are the especial physical signs of that particular affection.

Such are the situations in which, and the circumstances under which, the "Murmurs" arising from disease of the different valves are generally heard most distinctly. Such may be said to be the ordinary rule. But, whether the fact arise from an alteration in the relative situation of the different parts of the heart, produced by enlargement and distention, or from other causes, it is assuredly a rule liable to occasional exceptions, and to exceptions moreover which are not always easily to be explained by examination of the body after death. There are, however, many circumstances in connexion with other physical signs, and with the general symptoms, which tend very materially to facilitate the diagnosis of the diseases of the different valves; but upon these it is not now my province to dilate. 
Hitherto those murmurs which arise from the direct or forward progress of the blood have been alone referred to. But it often happens that the valves are so stiffened and crumpled, so contracted and shortened, or that the openings; which it is their peculiar province to guard, are so enlarged, that the valves are incompetent to close them; or that, as the result either of a rent or of ulceration, an aperture exists in one or other of the valves, which admits of the backward passage of the blood. Under any of these circumstances a portion of the blood, upon the occurrence of the diastole, returns into the cavity from which it had been propelled by the systole. If, then, the imperfection exists in the tricuspid or mitral valve, part of the blood returns into the auricles on the contraction of the ventricles; if the imperfection exists in the sigmoid valves, part of the blood returns into the ventricles on the contraction of the large vessels. In its return, the blood passes over a rough surface, or through a contracted space. The space may be absolutely contracted, or it may be contracted only comparatively with the size of the cavity to which it is the means of access. The backward passage of the blood, through this space, may, and frequently does, give rise to such agitation among the particles of the fluid, as to produce sounds similar to those which have been referred to as resulting from the direct passage of 
the blood through an obstructed valvular opening. There are, therefore, "Murmurs of regurgitation," as well as "Murmurs of obstruction."

Murmurs, then, may arise from the direct passage of the blood through a contracted valve; they may arise from the retrograde motion of the blood through an imperfect valve; or they may arise from both causes combined. When a murmur arises from the direct passage of the blood through one set of valves, and from the retrograde motion of the blood through another set of valves, though the cause be double, the murmur appears almost universally to be single, because they occur at the same time: that is to say, supposing a murmur to accompany the first sound of the heart, the systole of the ventricle, and to be synchronous with the pulse, it might be dependent either upon obstruction to the passage of blood through either the aortic or the pulmonary valves, or upon regurgitation of the blood through the mitral or tricuspid valves, or, indeed, upon both causes combined, as they all occur at, or nearly at the same time. When, on the contrary, a murmur arises from the direct passage, and also from the retrograde motion of the blood through the same valve, or set of valves, or the corresponding pair of valves, the murmur is double because it occurs at different times. Thus, supposing such disease to exist in the aortic valves as at once to cause obstruction to the onward 
progress, and to admit of regurgitation of the blood, and supposing each to produce a murmur;-as the one would accompany the first sound of the heart and the systole of the ventricle, and synchronize with the pulse, and the other would accompany the second sound of the heart and the diastole of the ventricle, and therefore be not synchronous with the pulse, the time of the two would necessarily vary, and a double murmur would be the consequence. The same observations are applicable to the pulmonary, and also to the mitral and the tricuspid valves; excepting that in the case of the last two valves the synchronism is just the reverse of that occurring in the aortic and the pulmonary valves: that is, the murmur arising from obstructive disease of the mitral and tricuspid valves would occur during the diastole of the ventricle, accompany the second sound of the heart, and would not synchronize with the pulse; while, on the contrary, the murmur arising from regurgitation through these valves would accompany the first sound, the systole of the ventricle, and consequently would be synchronous with the pulse at the wrist, and, as a matter of course, also with the impulse of the heart against the parietes.

If the student bear these simple observations in mind, and reflect upon the circumstances upon which they are founded; if he recollect the origin 
of most if not all murmurs, obstructive and regurgitative; and, at the same time, remember the action of the heart, and of its valves, he will easily understand the following illustrations, and will, I believe, be capable of unravelling most valvular murmurs. Exceptions of course will occur to him, as exceptions occur to the most experienced; but to the one as to the other they will be only the exceptions to a very general rule.

When regurgitation takes place through the aortic valves, the disease giving rise to which does not in any material degree produce obstruction to the direct current of the blood, a murmur is heard most distinctly over the sternum, opposite to the third rib; it occupies the time or place of, and often entirely conceals, the natural second sound of the heart; though the second sound may be sometimes indistinctly heard, together with it, between the cartilages of the second and third rib, upon the left side, resulting from the free and perfect action of the pulmonary valves.

When both obstruction exists in, and regurgitation takes place through, the aortic valves, a double murmur, or "see-saw" sound, is heard over the sternum, opposite the third rib, and passes upwards in the course of the aorta, but gradually decreases in power as the stethoscope is farther and farther removed from the situation of the valves. Should the same double murmur, or "see-saw" sound, 
occur opposite the second intercostal space,- - should the murmur be pretty much confined to that situation, and not follow in any degree the course of the aorta,-it may be presumed, rare as, comparatively speaking, is the affection, that obstruction exists in, and regurgitation takes place through, the pulmonary valves.

When a murmur which accompanies, or occupies the time and place of the first sound of the heart, is most distinctly heard below the nipple, is audible in the axilla, and in the left interscapular region close to the spine, or over the bony column itself; when it gradually diminishes in power as the stethoscope is removed from these localities; and when it is not at all, or only very imperfectly, conducted along the course of the aorta, it may be presumed that regurgitation takes place through the mitral valve. This regurgitation, however, it must ever be borne in mind, may occur through the mitral valve, and through other valves, in consequence either of the valves themselves being diseased and contracted, and thereby unfitted to perform their natural function of closing their respective apertures, or of the same apertures being dilated, and the valves, though healthy, being consequently incapable of doing so.

Supposing, then, that a loud murmur occupies the time of the first natural sound, is equally loud behind the sternum, opposite the third rib, and 
below the nipple; that it follows the course of blood in the aorta, and is also distinctly heard near the spine, opposite to, or rather below the angle of the scapula-what, according to the preceding statements, may be presumed to be the probable nature of the affection? If the student reflect, he will reply, "Probably direct obstruction of the aortic valves, and regurgitation through the mitral valve."

Supposing, again, that a murmur was heard occupying the time of the first sound of the heart, or the systole of the ventricle, situated over the aortic valves, and passing up, with the current of the blood, in the course of the aorta, and that a murmur was also heard in the same situation to accompany the diastole of the heart, and occupying the period of its second sound; according to the preceding statements it might be fairly, and in truth it might be correctly, assumed that there existed both obstruction and regurgitative disease of the aortic valves. The diagnosis would be of course confirmed by the "thrilling," "splashing," "hæmorrhagic," or "water hammer" pulse, so especially characteristic of an unfilled artery.

Once more: supposing a murmur to be heard over or about the second intercostal space, accompanying the systole of the ventricle, and that this murmur did not pass up in the course of the aorta, appeared to be very superficial or close to the ear, 
passed up a little to the left, and then abruptly terminated, it might be not unfairly assumed that, rare as it is, and in the absence of the evidence of anæmia, obstructive disease existed in the valves of the pulmonary artery.

It must surely be unnecessary to illustrate this subject farther. Let the student reflect upon these examples-let him, as it were, follow them homelet him inquire into, see, and fully appreciate, "the why and the wherefore" of each case, and I believe he will be capable of making out most of the instances of murmurs, or morbid valvular sounds, which occur under his notice.

He will be greatly facilitated in doing this by the recollection that valvular disease on the right side of the heart is exceedingly rare, comparatively with that of the left side; that regurgitative disease of the aortic valves is of frequent occurrence; and that a direct or obstructive murmur of the mitral valve is, to say the least, by no means common; and therefore that murmurs accompanying the diastole of the ventricles may be ordinarily attributed, with considerable assurance, to regurgitation through the aortic valves, and that those connected with the mitral valve may be deemed to arise, in the great majority of cases, from regurgitation.

These statements may be perhaps considered of sufficient importance, in reference to diagnosis, to 
be repeated in a somewhat different form. Valvular disease exists much more frequently upon the left than upon the right side of the heart; and, even when present upon the right side, almost always exists to a greater extent upon the left than upon the right side. Murmurs connected with the mitral valve arise much more frequently from regurgitation, than from obstruction to the direct passage of the blood. As a rule, therefore, though, like most other rules, it is liable to many exceptions, it may be generally considered that murmurs arising from valvular disease proceed from the left side of the heart; and that, if synchronous with the diastole of the ventricle, they arise from regurgitation of the blood through the aortic valves.

But do murmurs necessarily indicate either obstruction from valvular disease, or regurgitation from valvular imperfection? Is valvular disease always accompanied by murmurs? Both these questions must be answered in the negative.

Morbid sounds, resembling murmurs, may in the first place arise from diseases of the pericardium, which will be separately considered hereafter.

But as, in the case of the stream previously referred to for the purpose of illustrating morbid sounds, any solid substance of sufficient density, which encroaches upon the channel by projecting 
from its banks, may, like a solid body placed in the centre of the current, produce a strong ripple or eddy, and give rise to a liquid rustling sound; so any substance projecting inwards from the parietes of the heart, or from the coats of the large vessels, or so compressing them as materially to encroach upon the channel through which the blood passes, may give rise to an agitation of the fluid similar to that resulting from obstruction in the valves, and may, therefore, like it, give rise to a variety of murmurs.

Aneurisms, independently of the local pressure which they as tumours exert upon the heart, and other diseases of the aorta, may also give rise to murmurs.

When the stethoscope is pretty firmly pressed upon a large artery, as the abdominal aorta, the axillary, the femoral, or the carotid, so as partially and locally to obstruct, but not to stop, the current of the blood, a whizzing noise or bellows murmur, if the current be of sufficient momentum, is constantly heard, which is synchronous with the systole of the ventricle. This evidently arises from the increased agitation caused by the obstruction among the particles of the fluid, being communicated to the walls of the vessel, and thence through the stethoscope to the ear. Such a sound, created by the examiner himself, has often given rise, and may, with the incautious, still give rise, to the in- 
correct supposition of the presence of aneurism. It may, indeed, prove a source of fallacy to the most careful and circumspect. Nor can it be regarded as surprising that such should be the case, when it is recollected that the sound of an aneurism is often very similar, if not absolutely identical, and that it arises from a precisely similar cause, viz., obstruction to the current of the blood.

Like the pressure of the stethoscope, the pressure of a tumour, as of an abscess, or of malignant growth, upon the course of a large vessel, may produce a murmur, and may thus very exactly simulate an aneurism.

The mere dilatation of a vessel does not, I believe, give rise to a murmur; nor does the entrance of blood into an aneurismal pouch necessarily produce a whizzing noise. But if the dilated vessel have its lining membrane roughened; if it be studded with atheromatous or ossific matter; and if this roughening, or this deposit, exist to such an extent as materially to interfere with the free passage of the blood, then a turmoil, agitation, or eddying, is excited in the current, like that of a rapid stream with a rough bottom; and such vibrations of the coats of the vessel are produced, as constitute a bellows murmur, or sometimes a harsher sound.

If an aneurism press not upon the calibre of the vessel from which it arises, or upon that of any 
other vessel of large size; if the opening, by which the blood enters the pouch, be as large, and as direct, as that by which it passes along the parent trunk; if, in fact, there be no obstruction, direct or indirect, to the passage of the blood, no morbid sound will be produced. But if the converse of this exist; if, that is to say, the aneurism do press upon the vessel from which it arises, or upon some other vessel; if the opening by which it communicates with the artery be not large, or be oblique: so that obstruction does exist either to the direct passage of the blood through the parent trunk, or to its admission into the aneurismal sac arising from it, then will be produced the indications of such obstructions; a whizzing noise will then arise, or some other morbid sound will be excited.

Aneurism, or other disease of either the ascending aorta, or of the arch of the aorta, may, therefore, give rise to sounds very similar to, if not identical with, those arising from obstruction in the valves of the vessel. They may, however, be very generally distinguished by their position, by their superficial character, and by the general symptoms or local signs which result from pressure.

Aneurism of the descending, and even of the ascending aorta, may give rise to sounds below the nipple, or in the back, or in both situations, so as very exactly to resemble the morbid sounds arising 
from regurgitation through the mitral valve. The diagnosis in such cases is often a matter of extreme difficulty. It can, indeed, be rarely determined with positive certainty, unless the aneurism project externally, or other local signs exist which are incompatible with simple regurgitative disease of the mitral valve.

Ancemic Murmurs.-In certain states of the system, or, it may be, with certain conditions of the circulating fluid, as in chlorosis, or in anæmia from hæmorrhage or from other causes, murmurs frequently arise from the passage of the blood, independently of absolute disease of the heart or great vessels. These are termed ancemic murmurs, or, as they frequently coexist with chlorosis, chlorotic murmurs.

They are ordinarily of the softer kind, and resemble the blowing of a pair of bellows ("bruit de soufflet;") but they are sometimes harsh, and resemble the rougher morbid sounds, as that of filing or sawing ("bruit de râpe and bruit de scie.")

They are very generally supposed to be confined to the aortic openings. But, from their local character, $i$. $e$. from their not passing up with the current of the blood in the course of the aorta, from their abrupt termination on the left of the sternum, it appears probable that, oftentimes at least, they may be connected with the pulmonary artery, in 
which murmurs, quite independent of any disease of the vessel or of its valves, are far from uncommon.

Murmurs may, for example, arise from some body pressing upon this vessel; as a solid mass, the result of pleurisy, of pneumonia, or of phthisis, enlarged bronchial glands, abscesses of the anterior mediastinum, \&c. \&c. The murmurs of the pulmonary artery also, as I believe, independently of such pressure, or of disease of the vessel itself, frequently coexist with chlorosis, or with other forms of anæmia.

Are these latter murmurs, then, whether in the pulmonary artery, or in any other part of the circulating system, to be distinguished with tolerable certainty from morbid sounds, the result of organic obstruction within or without the heart or large vessels?

Generally speaking, they may, I believe, be distinguished from each other; but they assuredly cannot always be so; and never with absolute certainty from the character of the murmur alone. There are, I feel assured, some examples of these anæmic murmurs, which can be proved to be simply functional, and not to arise from organic disease of the heart or its vessels, or from pressure upon them, only by the results of treatment.

Let, then, the student be careful not to assert too confidently that a patient, on the one hand, has 
organic disease of the heart, or great vessels, merely because he or she has a murmur, and it may be a harsh murmur, over the aorta, an occasionally irregular rhythm, and a vibrating pulse, which usually coexist with an anæmic condition of the body; or he may cause unnecessary alarm and anxiety: nor let him, upon the other hand, too hastily determine, that because a murmur is soft, and his patient is an hysterical girl, with a pale face, and is subject to leucorrhœea, and to amenorrhœea, she has no organic disease; or some day, to his great surprise, grief, and mortification, and possibly also to his disgrace, he may find she has died suddenly with diseased heart.

Anæmic murmurs, however, it may be stated, are very local, and are generally pretty much confined to the situation of the sigmoid valves, either aortic or pulmonary, or both : they do not follow the course of the large vessels so fully, or so frequently, as do the murmurs arising from disease of the valves, or of the arteries; they occur only during the systole of the ventricles; and, as they cannot arise from regurgitation through the mitral valve, they are generally not heard below the left nipple; they are almost always accompanied with a smart smacking impulse: they generally disappear for a time while the individual is quiet, mentally as well as bodily, if by that quiet the heart assume a natural impulse; and they are always diminished, and ge- 
nerally disappear entirely, under suitable treatment.

The origin of these anæmic murmurs has latterly been very generally attributed to a watery condition, or a diminution of ordinary viscidity, of the blood; in consequence of which it is believed that the particles of the fluid move more easily over each other, are therefore more freely agitated, and thus give rise to the vibrations which produce the murmur. This may have some, and perhaps an important, influence in producing them.

But there are other circumstances which also appear to play an important part in their causation. The principal of these is the remarkably quick and sudden contraction of the ventricles; in consequence of which the fluid contents of the cavities are propelled through the comparatively small area of the mouths of the large arteries in a shorter time than during the leisurely contractions of health, or the frequent, but not sudden, contractions existing in some other forms of disease. Though, therefore, no actual contraction exists, an obstruction is practically produced by the increased velocity with which the blood is propelled through the aortic and pulmonary openings. The increased agitation in the fluid thence arising, it is at least probable, has a principal part in the production of àæmic murmurs.

If the heart beat quietly, and the impulse be 
natural, however decided the pallor of the face, and whatever the watery condition of the blood, no murmur, I believe, exists when no mechanical obstruction is present.

It is also possible that the quantity of the circulating fluid is decreased in such cases, in addition to its quality being altered, and that while, by the elasticity of their coats, the arteries are capable of accommodating themselves to the diminished quantity of the fluid, the cavities of the ventricles retain their normal capacity, and that on this account an absolute, as well as a comparative obstruction, may exist to the transit of the blood.

Venous Murmurs.-Concurrently with these anæmic murmurs at the origin of the large arteries, there is often heard, upon the light application of the stethoscope to the side of the neck, a curious sort of humming noise, which ceases both when the pressure of the stethoscope is either increased or greatly diminished, and also when firm pressure is exerted upon the jugular vein at a point above that on which the end of the stethoscope is placed. It is continuous, not intermittent like the arterial murmur, and is therefore sometimes called the "continuous humming," as well as the "venous murmur," and in consequence of the sound resembling that of a French toy- "bruit de diable," \&c.

It most probably depends upon partial obstruc- 
tion to the quickened flow of blood through the jugular veins. Strong pressure causes it to cease; but without pressure, directly or indirectly applied, it is, I believe, never heard. Like the anæmic murmur of the arteries, it is generally believed to be associated with a watery condition of the blood, and it is certainly a frequent, if not a constant, attendant upon that state of the system with which such a watery condition of the blood is a concomitant.

The influence of pressure in the production of, and the constant coexistence of, a watery or otherwise disordered state of the blood, with the venous murmur, has been recently questioned, if not denied, by more than one writer upon diseases of the chest, in consequence of its asserted presence, not unfrequently, behind the left sterno-clavicular articulation; in which case it is, as I think incorrectly, assumed that pressure can have no influence; and in consequence of its having been discovered in a very considerable proportion of healthy, or at any rate rosy, children submitted to examination. Of the venous murmur behind the left sternoclavicular articulation I have no experience; but multiplied observations convince me that in the neck its occurrence is intimately connected with pressure either directly or indirectly applied, and that, without pressure, directly or indirectly applied, it cannot be proved to exist at all. Multi- 
plied observations also induce me to believe that it is connected with a watery, or otherwise defective condition of the circulating fluid; as upon very many occasions I have observed it to diminish, and ultimately to cease altogether, upon the improved tone of the system, and the consequently assumed improvement in the quality of the blood. Notwithstanding, therefore, the above-mentioned opinions, I still fully believe that the existence of a well-marked venous murmur does indicate the presence of an impoverished condition of the blood, and that it does also indicate, according to the statement of a highly esteemed English physician of large experience, the admissibility, if not the desirableness, of the administration of chalybeate medicines.

For a more detailed account of my own opinions upon "Anæmic Murmurs and their diagnosis," I may refer the student to my paper upon this subject in the Guy's Hospital Reports for the year 1850.

But great obstruction to the blood may, as has been previously hinted, exist; extensive disease may be present in the valves of the heart, or in the large arteries, and yet no murmur may be heard. This arises from circumstances which may be farther, as they have already been partially, illustrated by the stream, in which a certain rapidity of the current is necessary to produce such 
an undulation or agitation of the water as will give rise to sound. Though the bottom of a rivulet be very uneven, and its banks exceedingly irregular; yet; if the current be not tolerably strong, little or no ripple will be produced, and no sound will be generated. It is just so with the blood; rapidity of the current of the blood, as well as obstruction thereto, is necessary to produce such an agitation among the particles of the fluid as will give rise to sound.

Hence it often happens that a heart with extensive disease of the valves may be without murmur while the patient is quiet, and the circulation is slow; though immediately the circulation is accelerated, either by physical exertion or by mental emotion, a murmur becomes distinct. Hence, also, it happens that when the cavities of the heart become greatly distended, in consequence either of the magnitude of the obstruction, of imperfect nutrition, or of defective nervous power, the ventricles are frequently incapable of acting upon and propelling their contents with sufficient force to produce a murmur. The channel may be irregular enough, but the rapidity of the current, and of the resulting vibrations, may not be equal to the generation of sound. Hence, likewise, it arises, that when fluid is present to a large amount in the pericardium, the heart may be so oppressed with the accumulation upon its exterior, that, though 
great obstruction exists within, no murmur is produced. Thus, what to the uninitiated may appear a curious anomaly, it may be often observed that when the obstruction is greatest, the murmur, if even it be heard at all, is very feeble; and that when the obstruction is small, the murmur is very loud: thus, also, it happens that in persons who, for weeks and months, and even years, have presented notably morbid cardiac sounds, these sounds, if the individuals are not carried off suddenly, very frequently, or perhaps even generally, cease altogether some days before death.

The causes of this, as before stated, are either that the heart does not contract with sufficient power, or, though it act forcibly, that it cannot act upon and propel through the contracted orifices the large quantity of blood, which distends its cavities, with a rapidity sufficient to give rise to such an amount of agitation in the fluid as to produce a murmur.

Let, then, the student ever bear in mind the important truth, that mere obstruction is not in itself sufficient, but that a certain force or rapidity of the circulation must be necessarily combined with that obstruction, to give rise to morbid endocardial sounds. Murmurs may exist without any obstruction of an organic kind; but without a certain degree of force or celerity in the circulating current, they cannot exist at all. 
Pericardial Murmurs.-The movements of the heart in the pericardium, when it is healthy, are not accompanied with any sound. The folds of the glistening and slippery membrane, like those of the pleura, glide over each other without noise. But when the surface of the membrane is rendered rough by inflammation-when solid or semi-solid plastic lymph is effused upon one or other of its folds-the smooth gliding motion ceases, attrition occurs, and a superficial rubbing noise is heard over the pericardial region upon each motion of the heart. This is "pericardial rubbing," "exocardial murmur," or "frottement."

It may exist in, and be confined to, a small space; or, though commencing locally, it may gradually extend over the entire surface; or it may simultaneously appear over the whole of the membrane at once.

As the fluid of pleurisy intervening between the two surfaces of the pleura removes pleuritic rubbing, so the intervention of fluid between the roughened surfaces of the pericardium prevents their attrition, and most commonly removes the rubbing sound.

Under such circumstances the sound may gradually disappear from below upwards, like that of pleurisy, as the fluid accumulates; and, after its entire cessation, it may gradually reappear from above downwards, as the fluid becomes absorbed, 
and may become again audible over the entire surface occupied by the pericardium.

When only a thin layer of fluid exists in the serous sac, the rubbing noise may, after its temporary cessation, be sometimes made to reappear in certain situations by altering the position of the body, and thus causing the effused fluid to gravitate to the lowest part of the bag, and to leave the uppermost surfaces comparatively dry. It may also, under similar circumstances, be occasionally reproduced by strong local pressure of the ear, but especially of the stethoscope; by which the two rough surfaces of the membrane are brought into contact by the interposing fluid being squeezed away from the part to which the pressure is applied. This fact, which has been recently brought forward as a novel discovery, has been for many years known and taught by myself and others.

After entire absorption of the fluid, the two layers of the membrane commonly adhere; if the adhesion be complete, the rubbing noise of course ceases. But sometimes the absorption is incomplete, or other causes prevent the adherence of the two layers of the membrane, the surfaces of which continue permanently roughened. The pericardial rubbing consequently remains behind, and, unless a fresh attack of inflammation occur, by means of which adhesion is produced, or a fresh effusion of fluid takes place, which separates one layer from the other, it becomes permanent. 
The character of the deposit, in the process of time, becomes altered. It may consist of nonplastic lymph; it may be converted into a hard, tough substance, approaching the consistence of fibro-cartilage, like the lining of a calf's mouth; or it may consist partially of fluid, and partially of cellular membrane. The sound which results from the attrition of the two surfaces varies accordingly. It may be a simple rubbing, "frottement;" it may resemble a harsh grating or scraping noise, "raclement;" it may simulate precisely the creaking of new leather, "cracquement de cuir ;" or, finally, its character may very nearly approach the bellows murmur of the valves, "bruit de soufflet."

Is, then, a pericardial sound always to be distinguished from an endocardial or valvular murmur? Can it always be positively stated-this is a pericardial rubbing, and that is an endocardial murmur? This is inside the heart, and that is outside the heart? By experienced auscultators it may indeed be generally so stated; but I believe not always. For myself, at least, I must confess that I am sometimes in doubt, and, moreover, sometimes wrong, in my conjectures upon this question. The pericardial rubbing occasionally so closely approximates, in character and situation, the valvular murmur; it appears, indeed, as regards the sound, so perfectly identical with it that I hesitate before I give an opinion, or I feel com- 
pelled to acknowledge my inability to form one. These are, however, exceptive cases, and they constitute very rare exceptions to a very general rule.

What, then, it may be inquired, are the especial characteristics of pericardial sounds? They are, or commonly appear to be, more superficial than even pulmonary or aortic murmurs; they approximate more to a rubbing or grating noise than the endocardial murmurs, which have more of a blowing or whiffing character; they ordinarily occupy the period, or a part of the period, of both the diastole and the systole of the ventricles, and very commonly also a portion of the interval or pause; they are, in fact, less decidedly intermittent than the valvular murmurs; they pass over the period of the natural sound, and leave an additional prolongation to be explained; and often afford the impression of a circular rubbing motion, like that of a mullar used in grinding paint, rather than of the puff or whizz of a valve; they are more localized-i. e., they extend beyond the surface, by the attrition of which they are produced, less than, or, in other words, not being produced by the agitation of the blood, do not follow the ceurse of the blood so obviously as do the valvular sounds.

Though, then, it may be acknowledged, that it is not possible to state with absolute certainty, in all cases of old standing disease, "This is a pericardial rubbing," and "That is an endocardial mur- 
mur;" yet the cases in which this difficulty occurs are on the whole rare; the sounds are ordinarily sufficiently distinct to a person with a moderately good ear. So distinct, indeed, are they on some occasions, that in cases of acute endocarditis accompanied with pericarditis, the experienced auscultator can often in the first place detect the endocardial murmur, and then the gradual supervention of pericardial rubbing, when the two sounds may be heard at the same time:-he may be able then to observe, that, as the pericardial effusion increases, the rubbing becomes so loud as to drown the feebler sound of the valves, - that it then gradually decreases as the plastic matter becomes partially absorbed, and that the two sounds are again heard in combination; till at length adhesion of the pericardium takes place, when the endocardial murmur becomes again, as it was in the first instance, pure and unalloyed.

I may yet repeat, though I believe they are comparatively few, that instances do occur in which it is quite impossible to discriminate, or with certainty to decide, between a pericardial rub, and an endocardial murmur.

Mixed Murmurs.-Independently of the combination of sounds, which has been just referred to, there are some other irregular sounds of the heart, which require a brief notice, and which have been 
deferred to this place, in consequence of their being dependent upon affections of other organs conjointly with the heart.

It occasionally happens, that, though the pericardium is adherent, or quite free from disease, the pleura which is in contact with its exterior becomes inflamed and roughened. Under such circumstances, though the motions of the heart, in its proper serous membrane, give rise to no noise, there does arise a noise from the attrition of the two surfaces of the roughened pleura; that is to say, of the fold of pleura covering the pericardium and of that covering the lung.

This sound cannot be always with certainty distinguished from the proper rubbing of pericarditis. It may, however, be sometimes distinguished by the following circumstances. When the lungs are inflated, they press with more force against the heart bag than when they contain a less amount of air. A fresh surface also of the pulmonary pleura is probably brought into contact with that covering the pericardium during inspiration. The consequence of this is, that the rubbing noise dependent upon the action of the heart against the roughened pleura, is sometimes only heard, and is generally most distinctly heard, during the inspiration. It possesses, therefore, a double synchronism. It keeps time with the respiration, and with the heart also; it disappears or diminishes during the expiration, 
and reappears or increases with each act of inspiration; yet, when occurring, it coincides with each pulsation of the heart. As to time, therefore, it synchronizes with the pulsation of the heart; while as to intensity it often also synchronizes with the respiration.

Similar to the preceding, in many respects, is another sound. This, however, differs in being internal or endocardial, while that, though not pericardial, is strictly speaking exocardial. It is a valvular sound, a pure bellow's murmur, "bruit de soufflet," which, though audible during the inspiration, and during the inspiration only, is perfectly synchronous with each systole of the ventricle. It is, as far as I have observed, almost always audible over the valves of the pulmonary artery. It may be supposed to arise from the small additional pressure, caused by the inflation of the lungs, being sufficient under peculiar circumstances to create such an obstruction to the progress and consequent agitation of the blood, as to give rise to a sound. But how, as it exists in some few cases, and in very few cases only, the simple inflation of the lung is capable of producing this effect, it appears difficult to understand.

It occasionally happens, also, when a gangrenous, a phthisical, or a pneumothoracic cavity is situated in the immediate vicinity of the heart, that by each 
impulse of the organ an agitation is produced in the gaseous and fluid contents of that cavity.

A few such cases only have occurred in my experience. In each, the sound was very peculiar, and in each the precise physical conditions, which after death were found to have given rise to the sound, were predicted during life. In each the noise was very similar. It occurred during the dilatation as well as during the contraction of the ventricles, and evidently arose from the impression made by the stroke of the heart upon the sides of the cavity, and the agitation produced by that impression in its mixed fluid and gaseous contents. The noise may be perhaps best compared to that produced by dashing a spoon into a basin of porridge, or of hasty pudding, and may be pretty accurately represented by the syllables "blob blobblob blob." Excepting under the circumstances above mentioned $I$ have never heard a similar sound produced by the heart. When once heard, I imagine it could not be afterwards mistaken; nor indeed do I believe that it could-be well mistaken, even though it had never been heard before. 


\section{CHAPTER VI.}

MENSURATION, OR EXAMINATION BY ADMEASUREMENT.

AltнобGH, when other means of physical diagnosis are employed,-when Inspection and Palpation in conjunction with Auscultation and Percussion, are made available for the purposes of investigation,-and when the examiner is himself capable of judging of the indications thereby afforded, Mensuration is of comparatively little importance as a mode of physical examination, and certainly the least useful to the bedside physician; there are, assuredly, some cases of disease in which it may be practised with advantage.

Of the mode of examining the comparative depth of the two sides of the upper part of the chest, in suspected, or in the early stage of, phthisis; by means of instruments, I must acknowledge that I have no experience. I have never employed them, because I have never felt the need of them; indeed, I cannot but believe that, with a quick and correct eye, and a sensitive touch, they are unnecessary. From my own experience of the older modes of mensuration, indeed, I am quite satisfied 
that a slight flattening or sinking of one or other of the infra-clavicular regions may be sufficiently obvious to the eye, and that the imperfect elevation of one side may be sufficiently appreciable by both the eye and hand, though neither may be indicated by the employment of those instruments.

But since the appearance of the former edition of this work several instruments have been introduced, which by their respective inventors are considered to be important aids in the diagnosis of the chest; and I think it but justice to those gentlemen, as well as to the student, that some notice of these instruments, however brief, should be here taken. At the same time it is necessary to state that I have no practical experience of their applicability. to cases of disease, and that I may, therefore, be not unfairly considered to be incapable of judging of their utility. But important as I can conceive they may have been, and may still become, as means of investigation in vital statistics and in physiological experiments, I cannot help expressing my belief that, to the practical physician at the bed-side, they are but imperfect substitutes for experience in the investigation of the nature of existing disease; that, even to those familiar with their use, they are as crutches to the feeble, which should be discarded as he gains strength; and that, to the vigorous and active, they are but as clogs and impediments to his free action and progress. 
I say this with all respect to the excellent, laborious, and accomplished physicians who claim to be their respective inventors; and I beg to repeat that I say it also without any individual experience of their use, and solely in reference to ordinary bed-side practice in the investigation of disease.

The Spirometer.-The first in order of time, and by far the most important of these instruments, is the Spirometer of Dr. Hutchinson, of which, and of his laborious investigations connected with which, a detailed account will be found in the "MedicoChirurgical Transactions" for the year 1846. The Spirometer is a complicated instrument; but it is essentially composed of a tube through which to expire, a delicately poised receiver which is elevated by each increment of expired air, and a graduated scale attached thereto, by which the amount of expired air is correctly measured. The individual to be examined is directed to "loose his vest, stand perfectly erect, with the head well thrown back, slowly and effectually to fill his chest with air, or to inspire as deeply as possible; then to put the ivory mouth-piece between his lips (standing in the same erect position,) and holding it there sufficiently tightly, as not to allow any breath to escape. He is then slowly to make the deepest expiration." By these means, certain allowances being made for temperature, and the "residual air" (determined by the results of numerous experiments previously 
undertaken) being added to the amount marked by the index, the actual capacity of the chest is supposed, and in the main, I believe, correctly supposed, to be ascertained.

But this is not all. Dr. Hutchinson, by a vast series of experiments upon persons of different heights, selected as healthy individuals, ascertained that, as a rule, the actual capacity of the chest was proportioned to the height of each healthy person examined. He hence by a laborious process arrived at the determination of what ought to be the actual capacity of the chest of each well-made individual commensurately with his height; and thereby in proportion to the amount of air capable of being expelled by a forcible expiration, he arrived at what he denominates the vital capacity of the chest, and accordingly decided upon the presence or absence of disease in the chest of each individual submitted to examination.

Such, as I believe, with some minor exceptions as to age and weight, is a brief and imperfect account of the Spirometer, and its applicability to the investigation of disease.

It will be at once seen, even supposing it to be correctly assumed that the instrument is capable of determining that no disease now exists, and that it may be therefore important as a negative evidence, that of the extent, and of the nature of existing disease, it affords little or no indication at 
all; and that it is only applicable to persons who are well-shaped and free from any deformity, congenital or acquired. With the highest admiration of the inventor for his laborious assiduity and artistic invention, I am compelled to admit that, suitable and important as I believe his instrument may in many instances become, and of much service, as it may be, to the examiners of Insurance Offices, and of aspirants to occupation in the public services, to membership in Clubs, \&c., I do also believe that it is not at all applicable to bed-side practice, or indeed to the ordinary class of cases occurring in chamber practice; and that, although it may sometimes be the means of prognosticating future ailments, it is utterly inefficient in enabling the physician to decide upon the extent, and much more upon the nature of existing disease.

The chest measurer.-This is an instrument invented by Dr. Sibson, and in his hands it has, as we are assured, been employed with very striking results.

So far as I am able to understand it from his plate and description in the volume of the "Medico-Chirurgical Transactions" for the year 1848, it consists of two horizontal bars and one perpendicular graduated bar. Upon the lower bar, covered with silk, the patient lies; the graduated perpendicular bar, indicating the depth of the chest, supports the superior bar, which is capable of being 
extended, deflected, and elevated to any required extent. To this superior horizontal bar or rod is appended a dial and rack. "The rack, when raised by the moving walls of the chest, moves by means of a pinion the index on the dial. One revolution of the index indicates an inch of motion in the chest; each division indicates the 100th of an inch." Such, so far as I am able to gather from his own description, is a brief account of the "Chest Measurer." Of the applicability of the instrument to indicate the motions of individual parts of the chest, the inventor speaks highly. In physiological experiments I can suppose it may be useful; but as an instrument of the diagnosis of disease I know nothing, and I have not been so fortunate as to meet with any, except the inventor, who does.

The Stethometer.-The least, as well as the last, but in its practical application at the bed-side perhaps the most important of these instruments, is the Stethometer of Dr. Quain. In a paper, with a copy of which he has obligingly favoured me, he describes it as follows. "The little instrument consists of a flat case, not unlike a watch-case: on its upper surface is a graduated scale and an index. A silk cord, which may be of sufficient length to surround one half, or the whole, circumference of the chest, passes through an aperture in one side of the case. This cord acts on the index. When 
the cord has been drawn out, or extended, for the space of one quarter of an inch, the point of the index will once traverse the circumference of the graduated dial. In other words, when the index has gone once round, it shows that the cord has been extended one quarter of an inch; and this is the measure of the part to which the instrument is applied. The dial is graduated or divided into fifty equal parts. Each of these parts, therefore, is equal to to the fiftieth part of a quarter of an inch; that is, to the 1-200th part of an inch. The index is farther capable of going round a second time, on an additional quarter inch of cord being drawn out. Hence, two revolutions of the index are equal to half an inch of movement.

"Mode of application.-It is evident, that if the instrument be so placed that extension be made upon the cord, the amount of extension will be shown by the movement of the index on the dial. For example, if the instrument is laid flat on the spine, and held in its place by the first and second finger of the left hand, whilst the cord is carried around the chest, and pressed on one of the ribs, or the sternum, by the fingers of the right hand, then, when the individual under examination expands the chest during inspiration, the amount of expansion will be communicated to the chord, and thus indicated on the dial. The chord may then be directed around the opposite side of the chest, 25 * 
and thus will be at once seen any difference which exists in the relative mobility of the two at the point of examination. It will, of course, be absolutely necessary in every examination, such as this, that corresponding parts of the chest be compared."

Such is the account given by Dr. Quain of his instrument, and its application; and thus far I agree with him in believing that it may be a useful instrument in the investigation of some cases of disease. For a further report of the instrument, and its supposed applicability to the diagnosis of disease, or rather to the movements of the chest, I must refer the student to the "London Journal of Medicine" of October 1, 1850.

Mensuration, however, according to my own individual experience, when Palpation and Inspection are applicable, and fairly applied by competent investigators, is principally available, as a means of diagnosis of diseases of the chest, in the case of inflammatory, or of other effusions into the pleura. It has been already stated more than once, that the first mechanical result of fluid effused into the pleura is to compress the spongy tissue of the lung; and that, supposing the lung to be free from adhesions to the costal pleura or the diaphragm, and to be otherwise healthy, as the quantity of fluid increases, a corresponding portion of air is usually squeezed out of the organ; till it is at length re- 
duced to a small dense mass closely pressed against the spine. The mediastinum is at the same time pushed over to the opposite side of the chest. It is then, but ordinarily not till then, that the less yielding parietes of the chest begin to be enlarged -it is then, therefore, but commonly not till then, that Mensuration becomes effective as a means of diagnosis of effusion into the pleura. One side of the chest, therefore, may be actually quite full of fluid, without any appreciable enlargement. It is not until it becomes what may be regarded as more than full, that the ribs begin to be expanded, or the intercostal spaces begin to bulge.

But it must be ever borne in mind, that one side of the chest may be enlarged without any fluid existing in the pleura,-as from aneurism, from malignant disease of the lung, enlargement of the liver, or of spleen, and from other morbid growths, as well as from local emphysema the product of local obstruction. It must also be remembered, that the pleura may, on the other hand, be quite full of fluid, and may yet be, not only not larger, but even smaller than the opposite or healthy side. After one or more attacks of pleurisy, a portion of the fluid may have been absorbed or evacuated; and, from the lung being firmly bound down by adhesion, and therefore incapable of expansion, the ribs may, from atmospheric pressure, have fallen in, and the side may consequently have decreased in size in 
proportion to the amount of fluid removed; and instead of the heart being pushed over to the side opposite to that in which the accumulation exists, it may be drawn over towards that accumulation. Thus it may happen-and of the fact I have seen many examples, - that the heart, contrary to ordinary precept and example, may be actually drawn to that side of the pleura which is literally filled with fluid, although that fluid be in small quantity.

Mensuration, then, should not only never be considered as a determinate mode of deciding upon the presence of fluid in the pleura, when taken alone; but it should ever be regarded as of comparatively little value in confirming the diagnosis of its presence, derived from other sources, or dependent upon other modes of exploration.

This is more especially necessary to be considered where the operation of paracentesis is contemplated. For if, in a case of urgent dyspnoea, threatening immediate suffocation, the cause of which is supposed to be connected with effusion into the pleura, the trochar were to be plunged into the larger side-considered, on account of its size alone, to be the diseased side-it would not unfrequently happen that the healthy side would be punctured; the not improbable result of which would be, that the healthy lung would collapse, by the admission of air into the pleura, and the patient would fall dead at the operator's feet. 
This has already happened more than once from a mistake in regard to percussion similar to that which is now contemplated in reference to mensuration. The more dull side was incorrectly supposed to be the diseased side, and to be filled with fluid; while, in truth, it possessed its natural degree of resonance, and only appeared to be dull in consequence of the tympanitic resonance of the opposite side; which resulted from pneumothorax, which was the real cause of distress. The trochar was introduced; the air rushed in; the healthy lung collapsed; and, that of the other side being already collapsed, the patients immediately dropped down dead. Hence arises the very important practical rule, never to introduce the trochar into one side of the chest, supposed to be diseased, unless it has been previously ascertained that the lung of the opposite side, if it be not absolutely healthy, is, at least, capable of carrying on the respiration.

It has been frequently stated, as if it were of constant occurrence, that the side is enlarged in pneumothorax. It is true that it may be enlarged; but it may be also smaller than natural, or it may be of the same dimensions as the opposite one. There is no rule which can be, in this respect, depended on.

Mode of measuring the chest.-The simplest and best method of measuring the comparative size of 
the two sides of the chest appears to be the following:-A piece of tape, or a graduated measure, is passed round that portion of the chest especially selected for admeasurement, and while the two. extremities of the tape are brought forward, it is marked with ink, or the figure is noted at the part at which it crosses the spine. The finger of an assistant keeps it fixed at that part; the ends are then pulled tight, and carefully marked upon the exact spot at which they meet in the centre of the sternum. The space between the marks at the ends, and in the centre of the tape, of course indicates the difference, if any, in the bulk of the two sides.

The examiner will be often much surprised to find how very little this difference really is, though to the eye the enlargement of one side appears to be very decided. He must be especially careful that the tape or measure is even and straight, as a slight twist, or a very little obliquity in its direction, may be sufficient to reduce to nothing the very trifling difference which may really exist.

If now the examiner be desirous to measure the expansibility, or the capability of expansion, of the two sides, he has only to request the assistant to keep his finger firmly pressed upon the mark of the tape upon the spine; to draw the ends moderately tight up to the marks as before; to hold them loosely under his finger, so that they may slip 
easily; and direct the patient to take a deep inspiration or two. The portion of tape or measure which slips beyond the mark on each side will be the extent of the absolute expansibility of each.

For this admeasurement of the comparative expansibility of the two sides, the stethometer of Dr. Quain appears to me admirably adapted. But whether it may be depended on as a means of deciding the expansibility of isolated parts of the chest, my own experience does not enable me to decide.

When, as it indeed' often happens, it is thought desirable to attempt to ascertain whether the process of absorption is going on, or whether the fluid is upon the increase-whether, in fact, the side is progressively getting larger or smaller-the tape should be carefully preserved for future use and comparison.

This, indeed, really appears to be one of the most important applications of mensuration, as regards effusion. Upon all future admeasurements, the tape will be an index, or a sort of tidegauge, of the amount of fluid in the pleura; or, at any rate, will correctly indicate the increase or diminution which has taken place in the comparative size of the two sides. Except in the case of effusions or morbid growths, I must confess that I have little confidence in Mensuration as a mode of determining the nature and extent of disease. 


\section{CHAPTER VII.}

SUCCUSSION, OR EXAMINATION BY SHAKING.

Succussion is by far the simplest as it is one of the oldest modes of examining the chest in disease.

When a cask containing beer, or other fluid, is really "full to the bung," or when a glass vial is filled with air alone, no sound is excited by their being shaken. But when the cask contains air as well as beer, or when there is a little water in the vial, a distinct noise is heard when they are respectively agitated, in consequence of the free motions of the different fluids over each other; the vibrations produced in which are communicated to the wood of the cask, or the glass of which the vial is made, and thence to the surrounding air. Similar facts are observed in disease.

When the pleura contains fluid alone, as in simple hydrothorax, whether it be in large or in small quantity, no sound results from shaking the body. When it contains only air, as in cases of simple pneumo-thorax, even violent agitation of the trunk is not accompanied with sound. But when, from 
any cause, air and fluid exists in the pleura at the same time; when, whether it arise from an empyema giving way, and partially emptying itself through, a bronchial tube, from a phthisical vomica bursting into the pleura, or from an opening with the external air being effected by gangrene of the lung, gas gains admission into the serous membrane, and pleuritis with effusion results therefrom; then a very well-marked sound, resembling the rattling, or splashing of fluid in a cask, may very generally be heard upon any violent agitation of the patient's body. This is Succussion. It is unnecessary to describe it farther. It is like the noise produced by shaking fluid in a cask. It can scarcely be mistaken.

Mode of effecting Succussion.- Sometimes the mere jerking of the body, or a sudden twist made by the patient himself, is sufficient to produce Succussion; and it may be generally made to appear by an assistant shaking the body, while the ear of the examiner is applied to the chest. The most common, though somewhat clumsy mode of eliciting the sound, is for the person examining to give the body a good shaking, and then to put the ear down to the chest. But as individuals suffering from the complaints with which pneumotharax is associated, are ordinarily feeble, and often greatly exhausted, this often causes distress. The least wearing, and, 
upon the whole, the most satisfactory mode of eliciting Succussion, is by pressing the ear firmly upon the parietes of the chest, and, while the ear is so pressed, encircling and firmly grasping the patient's body with one arm, swinging it gently to and fro, and then suddenly stopping the motion. When the sound is distinctly audible, this simple mode of proceeding will almost certainly produce it.

The sound of Succussion may differ in tone, as in distinctness, according to the amount of the air and fluid present in the cavity. It is loud, deep, and resonant, when the cavity is large, and when the quantity of air is comparatively great. It is more feeble and treble when the cavity is small, or when in a cavity of large size there exists only a small quantity of air.

As it not unfrequently happens in pneumothorax with effusion, particularly in the latter stages of the complaint, that the gas is either absorbed, or driven out of the pleura, in consequence of the gradual increase of the fluid effused, the succussion may gradually decrease in distinctness and resonance, and at length entirely disappear. This usually arises, not, as has been sometimes incorrectly supposed, in consequence of the aperture into the pleura being closed, for an aperture in the pleura, produced by disease, and giving rise to pneumothorax by the admission of air through the lung, 
very rarely closes at all, but in consequence of the serous membrane being quite full of fluid, and therefore incapable of admitting the presence of air.

But, it may be asked, is Succussion pathognomonic, or only a characteristic, of pneumothorax with effusion? It is certainly a characteristic, but, as certainly, it is not a pathognomonic sign of the disorder. It has been stated, that a very large vomica containing some fluid, together with the air with which such cavities are ordinarily filled, has on some very rare occasions been known to have given rise to Succussion, as well as to metallic tinkling. I have never myself known it to arise from such a cause, nor have I any recollection of meeting any individual who had himself heard it under such circumstances. But it is clear that such a case might occur, and it is probable that some cases have already occurred.

But I have frequently heard that which, without great caution, is exceedingly apt to lead to error.I mean the exactly similar sound which often arises from shaking the body, when the stomach contains a mixture of air and fluid. Both sounds are often heard and felt by the patient himself, and both are sometimes heard by persons standing near to him, without applying the ear to the chest. The best mode, and in some cases the only mode, of discriminating between the two, is to examine the patient frequently; when the stomach is empty, as well as 
when it is full, and before as well as after his meals. Other physical signs, and other general symptoms, and the history of the complaint, will of course materially contribute towards the diagnosis; though, without repeated and careful examinations, it is sometimes exceedingly difficult to arrive at certain conclusions upon the matter. 










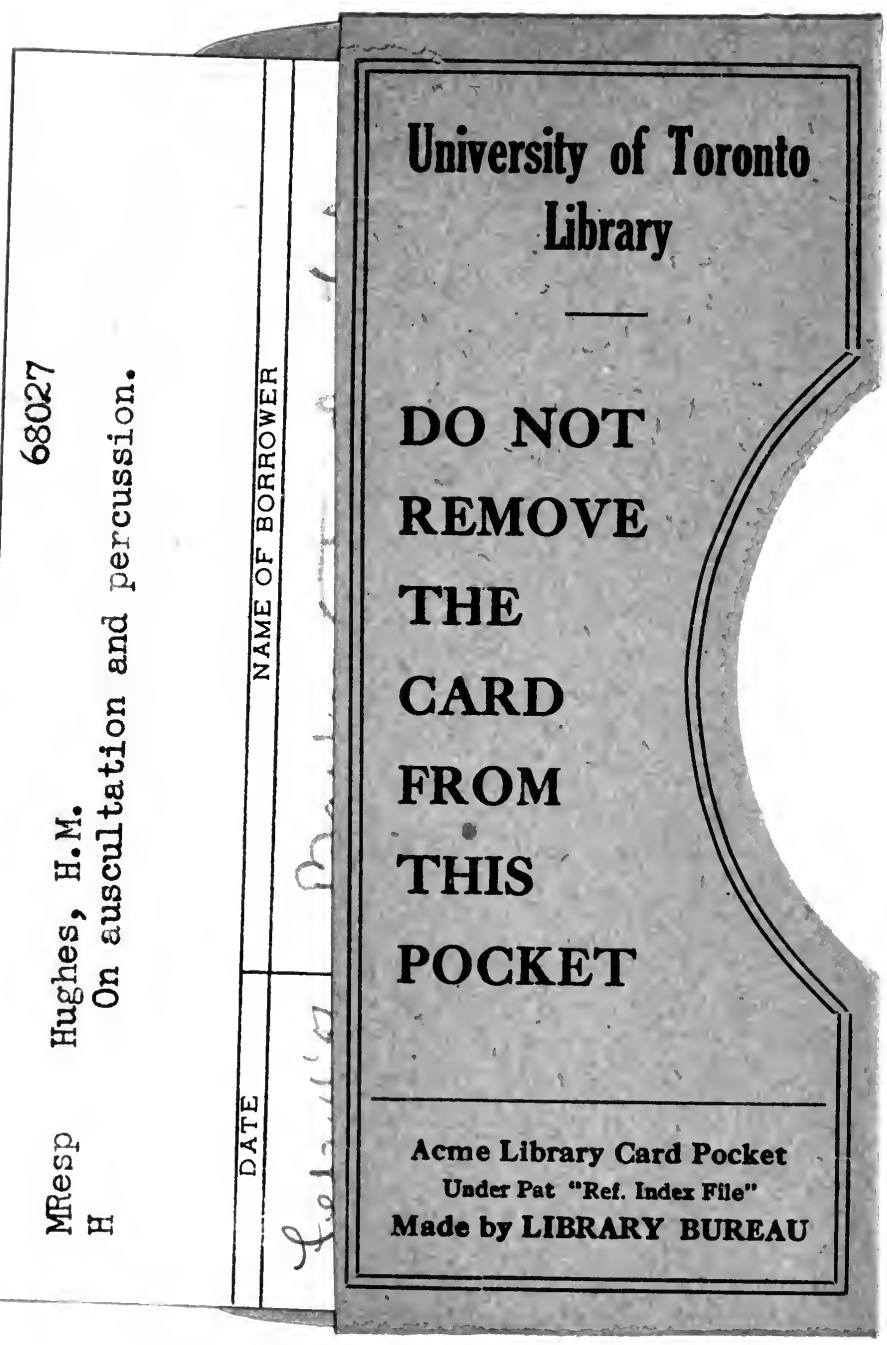


